\title{
DEA Malmquist productivity index based on a double-frontier slacks-based model: Iranian road safety assessment
}

\author{
S. S. Ganji and A. A. Rassafi
}

\begin{abstract}
Many governments in the developing world face the social and economic consequences of road accidents and mortalities. Hence, more precise evaluation of regional programs to reduce road fatalities has been a concern for many safety professionals.

Road safety performance is often measured using various extensions of Data Envelopment Analysis (DEA), in particular the model proposed by Charnes, Cooper and Rhodes (CCR), which deals only with the radial efficiency as the objective function neither taking into account input excesses nor output shortfalls. The Slacks-Based Measure (SBM) of efficiency overcomes this shortcoming by taking both measurements mentioned above simultaneously. In this regard, the current study aims to employ the SBM in analyzing road safety performance. It is noteworthy that the efficiency of each Decision Making Unit (DMU) can be pessimistically measured using the slacks-based measure of inefficiency such that the anti-efficient DMUs provide the anti-efficient frontier. The results obtained from the optimistic and pessimistic frontiers are nonlinearly aggregated by means of the Evidential Reasoning (ER) algorithm. Furthermore, a Double-Frontier SBM-based Malmquist Productivity Index (DF-SBM- MPI) is provided to analyze the efficiency and technological changes in safety performance from 2014 to 2016. For this purpose, the standard SBM and Super-SBM models are used to compute the optimistic Malmquist Productivity Index (MPI); similarly, the pessimistic MPI is determined by means of the inverted SBM and Super-SBM models. Finally, the obtained MPIs from the two different points of view are geometrically combined to obtain the overall MPI.
\end{abstract}

Keywords: Double-frontier slacks-based measurement, Iranian provinces, Road safety performance, ER algorithm, DF-SBM-MPI

\section{Introduction}

The World Health Organization (WHO) reported that about 1.25 million people annually perish due to road accidents. More often than not, accident victims are from low and middle income countries [1]. In other words, the global contribution of under developed countries to road fatalities is on the rise. Subsequently, road fatalities have recently become a social dilemma in under developed countries. According to the WHO, nearly 18,000 out of $77,447,168$ Iranians passed away due to road accidents from 21 March 2013 to 20 March
2014. This means that around 23.2 out of 100,000 people died as a result of road accidents from 2013 to 2014, which is significantly higher than the global average of 17.4 per 100,000 people [1]. As a result of road accidents, Iran lost about six per cent of its gross domestic product [1].

Road safety performance is usually defined as an indicator for assessing countries, states, or provinces in terms of reducing road safety risks with regard to the existing resources. The number of crashes, fatalities, and injuries are usually considered as the three most

\footnotetext{
* Correspondence: r.alizadehganji@gmail.com

Department of Transportation, Faculty of Engineering, Imam Khomeini International University, Qazvin, Iran
} 
common road safety risk indicators. In this regard, data availability is crucial in selecting the input data as well as road safety risk indicators. In addition, the definition of road safety performance will be different depending on the main purpose of road safety programs provided by governments or local authorities. In the current study, the most successful province in terms of road safety performance is a province that experiences a lower number of fatalities due to the less amount of investment.

Data Envelopment Analysis (DEA) method, originally proposed by Charnes, Cooper and Rhodes (CCR) in 1978 [2], has recently been widely used to assess road safety performance [3-15]. Based on the standard DEA, a Decision Making Unit (DMU) is recognized as an efficient DMU which generates either the maximum output levels with the given input levels or the minimum input levels with the given output levels. The CCR-based DEA model [2] uses a scalar measure to compute the efficiency of DMUs.

The main disadvantage of the CCR model is that it does not directly take into account the input excesses or output shortfalls (input/output slacks). Accordingly, an additive model was proposed by Charnes et al. (1985) to contend with this shortcoming; however it also lacks a scalar measure in the range of $[0,1][16]$. Subsequently, Tone (2001) developed a Slacks-Based Measure (SBM) of efficiency in order to take into account both scalar measure and inputs/outputs slacks simultaneously [17]. To the best of our knowledge, no studies have assessed road safety efficiency using SBM-based DEA model. Existing studies have optimistically assessed road safety performance using the traditional CCR model. In other words, each DMU is assessed based only on the distance from the efficient frontier, which is composed of all efficient DMUs. In this situation, a DMU closer to the efficient frontier is more efficient than those that are farther away. On the other hand, the anti-efficiency value of each DMU can be pessimistically measured as the distance from the anti-efficient frontier. Consequently, a DMU farther away from the anti-efficient frontier is more efficient than those that are closer. Obviously, the efficiency results obtained using the optimistic and pessimistic perspectives are not the same, more often than not. In this respect, the present study aims to investigate a double-frontier SBM model to achieve a more realistic evaluation of road safety performance. In this regard, a nonlinear method of integration, namely the ER approach, is employed to integrate the two points of view $[18,19]$.

In addition, this study is meant to further analyze Iranian road safety performance over a period of time. For this purpose, Malmquist Productivity Index (MPI) is used. Traditionally, MPI values are computed using the optimistic DEA model, but this indicator can be equivalently obtained by utilizing the pessimistic DEA model.
In this regard, a novel double-frontier MPI is proposed for a comprehensive evaluation of road safety performance over a three-year period of time.

The rest of the study is organized as follows:

Section 2 reviews the existing studies on road safety evaluation. Section 3 discusses the optimistic and pessimistic SBM models, followed by section 4 that briefly describes the ER approach. Afterwards, the optimistic, pessimistic and integrated MPIs are explained in section 5 . Section 6 evaluates Iranian road safety performance not only in each year but also over a period of time by respectively implementing the proposed methods, Double-Frontier SBM aggregated by ER algorithm (DF-SBM-ER) and Double-Frontier SBM-based Malmquist Productivity Index (DF-SBM-MPI). Section 6 respectively implements the proposed methods, DF-SBM-ER and DF-SBM-MPI, in order to practically assess Iranian road safety performance not only during each year but also over a period of time. Conclusions and remarks are finally presented in section 7 .

\section{Literature review}

This section surveys the studies previously carried out on road safety assessment using DEA models. In 2000, Odeck analyzed the productivity of 67 vehicle inspection stations over a two-year period of time (1989-1991), by utilizing an optimistic CCR-based MPI on the basis of an input-oriented model (Appendix 1) with one input (effective days of work) and four outputs including technical controls, usage controls, licensing and administration [3]. Afterwards, Odeck (2006) evaluated the safety performance of the 19 regional road agencies with the assumption of variable return to scale instead of constant return to scales [4]. Additionally, Odeck (2006) analyzed the productivity change of regional road agencies over a three-year period of time by means of an optimistic MPI based on the model proposed by Banker, Charnes and Cooper (BCC) [20] with only three outputs (technical, usage and safety belt controls), while no inputs were taken into account [4].

Hermans et al. (2008) examined the road safety performance of 27 European countries with respect to road accident fatalities using the CCR-based DEA model along with other four weighting methods. They calculated the road safety index for 21 European countries based on seven outputs (i.e., alcohol and drugs, protective systems, speed, vehicle, infrastructure daytime running lights and trauma care), but without input data. It is also found that the DEA model and road safety rank are highly correlated [5]. Hermans et al. [6] further evaluated the European countries in terms of road safety performance, taking into account the above-mentioned seven inputs and two undesirable outputs (number of crashes and fatalities). They came to the obvious conclusion that the 
inverted DEA model is more suitable for the available data set than economic issues [6].

In 2011, Shen et al. assessed the road safety performance of 19 European countries by developing both multiple-layer $\mathrm{CCR}$ and $\mathrm{BCC}$ models. They hierarchically categorized all inputs, consisting of 13 road user behaviors, into three layers. Similarly, they classified four defined outputs into two layers, namely injuries and crashes [7]. Also, Shen et al. (2012) assessed the road safety performance of 27 European countries with respect to three desirable inputs, inhabitants, passenger-kilometers and passenger cars, and only one undesirable output, fatalities, by developing a maximization programming model, called DEA-based Road Safety model (DEA-RS). The presented DEA-RS is simply obtained by inverting the traditional CCR model to make it more suitable for the defined data set. Although the DEA-RS was formulated as an output-oriented model (Appendix 1.2 ), it can also be converted to an input-oriented model, by minimizing the weighted sum of the outputs rather than maximizing the weighted sum of the inputs [8]. In 2013, Shen et al. further evaluated the road safety performance of European countries over a ten-year period of time. For this purpose, they developed a DEA-RS based MPI [9].

In 2013, Egilmez et al. analyzed 50 U.S. states in terms of road safety performance, using an MPI based on the standard input-oriented CCR model with seven inputs (highway safety expenditures, registered vehicles, licenced drivers, vehicle-miles traveled, total road length, overall road condition and safety belt usage) and one output (fatal crashes). They normalized the data set to fit the CCR model. Accordingly, the input data was reduced to five inputs. In addition, the ratio of the total annual time to the fatality rate was introduced as the model's output [10]. Also, in 2015, Shen et al. developed the DEA-RS models with weight restrictions based on either shadow price or a priori knowledge. Then, they employed the proposed models to assess 10 European countries in terms of road safety performance with respect to two outputs, serious injuries and fatalities [11]. All input variables were also defined in the same way as those previously introduced by Shen et al. $[8,9]$.

Bastos et al. (2015) analyzed the 27 Brazilian states by utilizing a multiple layer DEA method, taking into account two main indicators, namely mortality and fatality rates. They pointed out the obvious fact that the number of fatalities should be used as undesirable output in road safety studies [12]. In this regard, their model was presented based on the inverse of the DEA model provided by Cherchye et al. [21].
Rosic et al. (2017) assessed the 27 Serbian police departments on road safety performance using the DEA and TOPSIS (Technique for Order Preference by Similarity to an Ideal Solution) methods. It is worth noting that they applied the DEA models previously presented by Hermans et al. [5] and Shen et al. [8] as well as the corresponding cross-efficiency methods. The number of fatalities and seriously injured people were also defined as two undesirable outputs [13].

Behnood et al. evaluated the Iranian road safety performance using a CCR model that is, in fact, an inverted input-oriented CCR model (Appendix 1), which mathematically defines an efficient DMU as a DMU with less output and more input. However, the input variables seem not to be appropriate for the presented model, since all authorities actually prefer to reduce the fatality rate with the least number of defined inputs, i.e. police operation, emergency medical services, etc. They also measured the efficiency of road safety performance based on a data set of 60 DMUs, including 30 DMUs in 2008, and 30 DMUs in 2009. They did not analyze the road safety performance over a period of time, although they utilized two-year data sets [14].

The literature review reveals that no study has assessed road safety performance using SBM model. In contrast to standard DEA model, SBM assesses road safety performance by considering both input excess and output shortfall. In addition, all previous studies analyzed road safety performance based on the efficient frontier while ignoring the anti-efficient frontier. However, research on double-frontier DEA models has recently been of interest to many researchers [22-24]. Therefore, the main goal of this study is to bridge this gap by analyzing road safety performance based on a double frontier SBM.

\section{Methods}

\subsection{Slacks-based measure (SBM) approach}

This section briefly describes the optimistic and pessimistic SBM model. The optimistic SBM model was proposed by Tone in 2001 [17]. Suppose that there is an evaluation problem consisting of $n$ DMUs with $m$ inputs and $s$ outputs respectively defined by $X$ $=x_{i j} \in \mathcal{R}^{m \times n}$, and $Y=y_{r j} \in \mathcal{R}^{s \times n}$. The production possibility set can be defined as follows:

$$
P=\{(x, y) \mid x \geq X \lambda, y \leq Y \lambda, \lambda \geq 0\}
$$

where all data sets are assumed to be positive. The optimistic SBM is mathematically expressed as the following fractional programming model: 
$\operatorname{Min} \rho_{\text {optimistic }}=\frac{1-(1 / m) \sum_{i=1}^{m} s_{i}^{-} / x_{i 0}}{1+(1 / s) \sum_{r=1}^{s} s_{r}^{+} / y_{r 0}}$

Subject to

$$
\begin{array}{ll}
x_{i 0}=\sum_{j=1}^{n} x_{i j} \lambda_{j}+s_{i}^{-}, & i=1, . ., m \\
y_{r 0}=\sum_{j=1}^{n} y_{r j} \lambda_{j}-s_{r}^{+}, & r=1, . ., s \\
\lambda_{j} \geq 0, \quad s_{i}^{-} \geq 0, \quad s_{r}^{+} \geq 0 . &
\end{array}
$$

where the input excess and output shortfall are respectively represented by slacks $s_{i}^{-} \in \mathcal{R}^{m}$ and $s_{r}^{+} \in \mathcal{R}^{s}$. The SBM model (2) minimizes both input and output inefficiencies, which are respectively defined by the mean rate of input reductions, $(1 / m) \sum_{i=1}^{m}\left(x_{i 0}-s_{i}^{-}\right) / x_{i 0}$, as well as the inverted mean rate of output expansions, $\left[(1 / s) \sum_{r=1}^{s}\left(y_{r 0}+s_{r}^{+}\right) / y_{r 0}\right]^{-1}$ (Tone, 2001).

By multiplying a positive scalar variable $q>0$ by the denominator and the numerator of the objective function of the fractional program (2) and making some adjustments, the linear program (3) can be achieved as follows:

$$
\operatorname{Min} \tau_{\text {optimistic }}=q-\frac{1}{m} \sum_{i=1}^{m} S_{i}^{-} / x_{i 0}
$$

Subject to

$$
\begin{array}{lr}
1=q+(1 / s) \sum_{r=1}^{s} S_{r}^{+} / y_{r 0} & \\
q x_{i 0}=\sum_{j=1}^{n} x_{i j} \Lambda_{j}+S_{i}^{-}, & i=1, . ., m \\
q y_{r 0}=\sum_{j=1}^{n} y_{r j} \Lambda_{j}-S_{r}^{+}, & r=1, . ., s \\
\Lambda_{i} \geq 0, \quad S_{i}^{-} \geq 0, \quad S_{r}^{+} \geq 0, & q>0 .
\end{array}
$$

where $\Lambda_{j}=q \lambda_{j}, S_{i}^{-}=q s_{i}^{-}$and $S_{r}^{+}=q s_{r}^{+}$. The optimal solution of model (3) is $\left(\tau^{*}=\rho^{*}, q^{*}, \Lambda_{j}^{*}, S_{i}^{-*}, S_{r}^{+*}\right)$. Consequently, the optimal solution of (2) is as follow: $\left(\rho^{*}=\tau^{*}, \lambda_{j}^{*}=\Lambda_{j}^{*} / q^{*}, s_{i}^{-*}=S_{i}^{-*} / q^{*}, s_{r}^{+*}=S_{r}^{+*} / q^{*}\right) . \quad \mathrm{A}$ DMU is efficient if and only if $\rho_{\text {optimistic }}^{*}=\tau_{\text {optimistic }}^{*}=1$. Such a condition can be obtained when there is no input excess, $s^{-*}=0$, and output shortfall, $s^{+*}=0$; otherwise, the DMU is defined as inefficient.

Taking the pessimistic SBM into account, the following production possibility set can be introduced:

$$
P=\{(x, y) \mid x \leq X \lambda, y \geq Y \lambda, \lambda \geq 0\}
$$

The anti-efficiency of each DMU can be mathematically formulated as follows:

$$
\operatorname{Max} \rho_{\text {pessimistic }}=\frac{1+(1 / m) \sum_{i=1}^{m} s_{i}^{+} / x_{i 0}}{1-(1 / s) \sum_{r=1}^{s} s_{r}^{-} / y_{r 0}}
$$

Subject to

$$
\begin{array}{ll}
x_{i 0}=\sum_{j=1}^{n} x_{i j} \lambda_{j}-s_{i}^{+} & i=1, . ., m \\
y_{r 0}=\sum_{j=1}^{n} y_{r j} \lambda_{j}+s_{r}^{-} & r=1, . ., s \\
\lambda_{j} \geq 0, \quad s_{i}^{+} \geq 0, \quad s_{r}^{-} \geq 0 . &
\end{array}
$$

The pessimistic SBM model (5) maximizes both the mean expansion rate of inputs, $(1 / m) \sum_{i=1}^{m}\left(x_{i 0}+s_{i}^{+}\right) / x_{i 0}$, and the inverted mean reduction rate of outputs, $\left[(1 / s) \sum_{r=1}^{s}\left(y_{r 0}-s_{r}^{-}\right) / y_{r 0}\right]^{-1}$. The fractional program (5) can also be converted to the following linear program (6) in the same way as the optimistic SBM.

$$
\operatorname{Max} \tau_{\text {pessimistic }}=q+\frac{1}{m} \sum_{i=1}^{m} S_{i}^{+} / x_{i 0}
$$

Subject to

$$
\begin{array}{lr}
1=q-(1 / s) \sum_{r=1}^{s} S_{r}^{-} / y_{r 0} & \\
q x_{i 0}=\sum_{j=1}^{n} x_{i j} \Lambda_{j}-S_{i}^{+} & i=1, . ., m \\
q y_{r 0}=\sum_{j=1}^{n} y_{r j} \Lambda_{j}+S_{r}^{-} & \\
\Lambda_{j} \geq 0, \quad S_{i}^{-} \geq 0, \quad S_{r}^{+} \geq 0, & q>0 . . ., s
\end{array}
$$

All parameters and variables are the same as those of the optimistic SBM. A DMU is anti-efficient if and only if $\rho_{\text {pessimistic }}^{*}=\tau_{\text {pessimistic }}^{*}=1$. Such a condition indicates that the relative DMU is located on the anti-efficient frontier; hence both slack values are zero.

\subsection{Evidential Reasoning (ER) algorithm}

The ER algorithm was originally introduced by Yang and Singh in 1994 [25], based on the theory of evidence proposed by Dempster in 1967 [26] and improved by Shafer in 1976 [27]. Suppose that there is a frame of discernment, $\Theta=\left\{H_{1}, \ldots, H_{\mathrm{N}}\right\}$, that contains a set of collectively exhaustive and mutually exclusive propositions. Mass functions (or basic probability assignments) are defined as follows:

$$
\begin{aligned}
& m: 2^{\Theta} \rightarrow[0,1] \\
& m(\Phi)=0 \text { and } \sum_{A \in \Theta} m(A)=1
\end{aligned}
$$

where $m(A)$ represents a belief degree in the interval $[0,1]$, assigned to subset $A$, and $\Phi$ is the empty set. The power set of $\Theta$ is expressed by $2^{\Theta}$. The ER algorithm aggregates the independent evidence, $m_{1}$ and $m_{2}$, based on the Dumpster's rule of combination, as follows: 


$$
\begin{aligned}
& {\left[m_{1} \oplus m_{2}\right](C)= \begin{cases}0, & C=\Phi \\
\frac{1}{k} \sum_{A \cap B=C} m_{1}(A) m_{2}(B), & C \neq \Phi\end{cases} } \\
& k=1-\sum_{A \cap B=\Phi} m_{1}(A) m_{2}(B)
\end{aligned}
$$

where $k$ is a normalization factor. Note that the Dumpster's rule is not compatible in such a situation with thorough conflict between evidence, $\sum_{A \cap B=\Phi} m_{1}(A)$ $m_{2}(B)=1$. In addition, many studies also pointed out that the Dumpster's rule of combination may be inappropriate to deal with the problems with conflicting evidence. In other word, in such a situation, the results might be counter-intuitive, irrational and complex [28-30].

In this regard, the ER algorithm has been proposed to effectively deal with the aforementioned shortcomings. Many studies were carried out based on the ER algorithm as an appropriate method of aggregation in dealing with certain and uncertain decision making problems [15, 25, 31-40].

Suppose that we intend to evaluate a decision making problem with $n$ DMUs using $G$ assessment grades as the frame of discernment, $\Theta=\left\{H_{1}, \ldots, H_{g}\right.$, $\left.\ldots, H_{G}\right\}$. It is also assumed that each DMU is assessed according to $L$ pieces of evidence, $E=\left\{e_{1}\right.$, $\left.\ldots, e_{l}, \ldots, e_{L}\right\}$, and $L$ relative weights, $w=\left(\omega_{1}, \ldots, \omega_{l}\right.$, $\left.\ldots, \omega_{L}\right)$. The mathematical expression of each DMU evaluation based on a certain evidence, $e_{l}$, is as follows:

$$
S\left(e_{l}\right)=\left\{\left(H_{g}, \beta_{g, l}\right), g=1, \ldots, G\right\}, l=1, \ldots, L
$$

where $\beta_{g, l}$ is a belief degree assigned to $H_{g}$ with respect to evidence $e_{l}$. Note that the sum of all beliefs is equal to unity, $\sum_{g=1}^{G} \beta_{g, l}=1$, in the certain environment and is less than unity in the uncertain conditions, $\sum_{g=1}^{G} \beta_{g, l}<1$ . In brief, $\sum_{g=1}^{G} \beta_{g, l} \leq 1$. Accordingly, the mass functions, including the assigned and unassigned probabilities respectively denoted by $m_{g, l}$ and $m_{\Theta, l}$, are calculated by multiplying the belief degree, $\beta_{g, l}$, by the relative importance degree, $\omega_{l}$, as follows :

$$
\begin{aligned}
& m_{g, l}=\omega_{l} \beta_{g, l}, g=1, \ldots, G, l=1, \ldots ., L \\
& m_{\Theta, l}=1-\sum_{g=1}^{G} m_{g, l}=1-\omega_{l} \sum_{g=1}^{G} \beta_{g, l}, l=1, \ldots ., L
\end{aligned}
$$

In addition, the unassigned probability mass, $m_{\Theta, i}$, can be derived using Eqs. (12) and (13):

$$
\bar{m}_{\Theta, l}=1-\omega_{l}, l=\quad 1, \ldots ., L
$$

$$
\tilde{m}_{\Theta, l}=\omega_{l}\left(1-\sum_{g=1}^{G} \beta_{g, l}\right), l=1, \ldots \ldots, L
$$

where $\bar{m}_{\Theta, l}$ and $\bar{m}_{\Theta, l}$ represent the relative importance of evidence $e_{l}$ and the ignorance respectively.

The ER algorithm aggregates probability masses using Eqs. (14) -(16) as follows:

$$
\begin{aligned}
& \left\{H_{g}\right\}: m_{g}=k\left[\begin{array}{c}
\prod_{l=1}^{L}\left(m_{g, l}+\bar{m}_{\Theta, l}+\tilde{m}_{\Theta, l}\right)- \\
\prod_{l=1}^{L}\left(\bar{m}_{\Theta, l}+\tilde{m}_{\Theta, l}\right)
\end{array}\right], g=1, \ldots, G, \\
& \left\{H_{\Theta}\right\}: \tilde{m}_{\Theta}=k\left[\prod_{l=1}^{L},\left(\bar{m}_{\Theta, l}+\tilde{m}_{\Theta, l}\right),-\prod_{l=1}^{L}, \bar{m}_{\Theta, l}\right], \\
& \left\{H_{\Theta}\right\}: \bar{m}_{\Theta}=k\left[\prod_{l=1}^{L}, \bar{m}_{\Theta, l}\right],
\end{aligned}
$$

and the normalization factor, $k$, is calculated using Eq. (17):

$$
k=\left[\sum_{g=1}^{G}\left(\prod_{l=1}^{L}\left(m_{g, l}+\bar{m}_{\Theta, l}+\tilde{m}_{\Theta, l}\right)\right)-(G-1) \prod_{l=1}^{L}\left(\bar{m}_{\Theta, l}+\tilde{m}_{\Theta, l}\right)\right]^{-1}
$$

In addition, Yang and $\mathrm{Xu}$ proposed the recursive ER algorithm as a novel aggregation process as follows [32]:

$$
\begin{aligned}
\left\{H_{g}\right\} & : m_{g, I(l+1)} \\
& =K_{I(l+1)}\left(m_{g, I(l)} m_{g, l+1}+m_{g, I(l)} m_{\Theta, l+1}+m_{\Theta, I(l)} m_{g, l+1}\right) \\
g & =1, \ldots ., G
\end{aligned}
$$

$$
\begin{aligned}
\left\{H_{\Theta}\right\} & : \tilde{m}_{\Theta, I(l+1)} \\
& =K_{I(l+1)}\left(\tilde{m}_{\Theta, I(l)} \tilde{m}_{\Theta, l+1}+\bar{m}_{\Theta, I(l)} \tilde{m}_{\Theta, l+1}+\tilde{m}_{\Theta, I(l)} \bar{m}_{\Theta, l+1}\right)
\end{aligned}
$$

$$
\left\{H_{\Theta}\right\}: \bar{m}_{\Theta, I(l+1)}=K_{I(l+1)}\left(\bar{m}_{\Theta, I(l)} \bar{m}_{\Theta, l+1}\right)
$$

The normalization factor, $K_{I(l+1)}$, is computed as follows:

$$
K_{I(l+1)}=\left[1-\sum_{t=1}^{G} \sum_{\substack{k=1 \\ k \neq t}}^{G} m_{t, I(l)} m_{k, l+1}\right]^{-1}, l=1, \ldots, L-1
$$

The ultimate assessment distribution can be achieved using Eqs. (22) and (23): 


$$
\begin{aligned}
& \left\{H_{g}\right\}: \beta_{g}=\frac{m_{g, I(L)}}{1-\bar{m}_{\Theta I(L)}}, g=1, \ldots, G, \\
& \left\{H_{\Theta}\right\}: \beta_{\Theta}=\frac{\tilde{m}_{\Theta, I(L)}}{1-\bar{m}_{\Theta, I(L)}},
\end{aligned}
$$

where $\sum_{g=1}^{G} \beta_{g}+\beta_{\Theta}=1$.

\subsection{Malmquist Productivity Index (MPI)}

Sten Malmquist proposed a quantity index to analyze input consumptions [41]. Fare et al. (1992) suggested a DEA-based MPI to measure the efficiency and technical changes on the basis of two measurements, namely efficiency and the productivity, that were previously proposed by Farrell (1957) and Cave et al. (1982) [42-44]. Subsequently, the DEA-based MPI was successfully applied in many studies to examine the productivity change regarding each DMU over time $[3,10,45,46]$. Wang and Lan recently proposed the integrated MPI as a geometric mean of both optimistic and pessimistic MPIs [47]. They pointed out that the double-frontier MPI measures the productivity changes more comprehensively than the traditional MPI, as all information is taken into account.

Although most of the carried out studies used the CCR model to construct the MPI; Liu and Wang employed SBM to compute MPI [48]. Therefore, a novel DF-SBM-MPI is proposed to thoroughly analyze the productivity changes, taking into accounts both optimistic and pessimistic points of view simultaneously. In this regard, the pessimistic SBM-based MPI (represented by PMPI) is computed along with the optimistic SBM-based MPI (represented by OMPI); thereafter, the MPIs are geometrically combined to generate DF-SBM-MPI.

The OMPI of each DMU can be achieved as follows:

$$
O M P I_{0}=\left[\frac{D_{0}^{t}\left(x_{0}^{t+1}, y_{0}^{t+1}\right)}{D_{0}^{t}\left(x_{0}^{t}, y_{0}^{t}\right)} \cdot \frac{D_{0}^{t+1}\left(x_{0}^{t+1}, y_{0}^{t+1}\right)}{D_{0}^{t+1}\left(x_{0}^{t}, y_{0}^{t}\right)}\right]^{1 / 2}
$$

where $\left(x_{0}^{t}, y_{0}^{t}\right)$ and $\left(x_{0}^{t+1}, y_{0}^{t+1}\right)$ denote the input and output data sets relative to time periods $t$ and $t+1$ respectively. $D_{0}^{t}\left(x_{0}^{t}, y_{0}^{t}\right)$ and $D_{0}^{t+1}\left(x_{0}^{t+1}, y_{0}^{t+1}\right)$ respectively represent the optimistic efficiency scores obtained in time periods $t$ and $t+1$, based on the data set relative to the same time period. $D_{0}^{t+1}\left(x_{0}^{t}, y_{0}^{t}\right)$ denotes the optimistic efficiency value in time period $t$, on the basis of the data set relative to time period $t+1$ and similarly $D_{0}^{t+1}\left(x_{0}^{t+1}, y_{0}^{t+1}\right)$ measures the relative efficiency in time period $t+1$, according to the data set relative to time period $t$.

It is also noteworthy that the $O M P I_{0}$ can be achieved by multiplying the optimistic efficiency change (denoted by $O E C$ ) by the optimistic technical change (denoted by $O T C$ ), as follows [44]:

$$
\begin{aligned}
\text { OMPI }_{0} & =\frac{D_{0}^{t+1}\left(x_{0}^{t+1}, y_{0}^{t+1}\right)}{D_{0}^{t}\left(x_{0}^{t}, y_{0}^{t}\right)} \times\left[\frac{D_{0}^{t}\left(x_{0}^{t}, y_{0}^{t}\right)}{D_{0}^{t+1}\left(x_{0}^{t}, y_{0}^{t}\right)} \cdot \frac{D_{0}^{t}\left(x_{0}^{t+1}, y_{0}^{t+1}\right)}{D_{0}^{t+1}\left(x_{0}^{t+1}, y_{0}^{t+1}\right)}\right]^{\frac{1}{2}} \\
& =O E C_{0} \times \text { OTC }_{0}
\end{aligned}
$$

$O E C_{0}>1$ demonstrates an improvement in the efficiency of $D M U_{0}$ over time period $t$ to $t+1$, whereas $O E C_{0}<1$ indicates that $D M U_{0}$ experienced a diminution in its efficiency. $O E C=1$ denotes no efficiency change over time period $t$ to $t+1$. Similarly, $O T C_{0}>1$ indicates that $D M U_{0}$ achieved a technical progress over time period $t$ to $t+1$, while $O T C_{0}<1$ shows that $D M U_{0}$ experienced a technical regression. $O T C_{0}=1$ expresses no technical change over time period $t$ to $t+1$.

All required efficiency scores are computed using the optimistic and pessimistic SBM models (3) and (6), rather than only the optimistic CCR model. The required programming models for computing two single period measures, $D_{0}^{t}\left(x_{0}^{t}, y_{0}^{t}\right)$ and $D_{0}^{t+1}\left(x_{0}^{t+1}, y_{0}^{t+1}\right)$, as well as two mixed period measures, $D_{0}^{t}\left(x_{0}^{t+1}, y_{0}^{t+1}\right)$ and $D_{0}^{t+1}\left(x_{0}^{t}, y_{0}^{t}\right)$, are in detail presented in Appendix 2.1. Obviously, $D_{0}(\cdot)=1$ means that the $D M U_{0}$ is efficient in a given time period, $t$ or $t+1$, with respect to the given data set, $\left(x_{0}^{t}, y_{0}^{t}\right)$ or $\left(x_{0}^{t+1}, y_{0}^{t+1}\right)$. In such a situation, the optimistic Super-SBM model, as presented in Appendix 3.1, is used for further evaluation of efficient DMUs, with $\rho_{\text {optimistic }}^{*}=\tau_{\text {optimistic }}^{*}=1$ [49]. For this reason, two single and two mixed period measures, called $\tilde{D}_{0}(\cdot) \geq 1$, can be driven as illustrated in Appendix 4.1.

The $P M P I_{0}$ regarding $D M U_{0}$ can be similarly computed by substituting the pessimistic period measures, $\left\{d_{0}^{t}\left(x_{0}^{t}, y_{0}^{t}\right), d_{0}^{t+1}\left(x_{0}^{t+1}, y_{0}^{t+1}\right), d_{0}^{t}\left(x_{0}^{t+1}, y_{0}^{t+1}\right), d_{0}^{t+1}\left(x_{0}^{t}, y_{0}^{t}\right)\right\}$, in Eq. (25) which can be achieved by multiplication of the pessimistic efficiency and technical changes, respectively called $P E C_{0}$ and $P T C_{0}$, as follows:

$$
\begin{aligned}
\text { PMPI }_{0} & =\frac{d_{0}^{t+1}\left(x_{0}^{t+1}, y_{0}^{t+1}\right)}{d_{0}^{t}\left(x_{0}^{t}, y_{0}^{t}\right)} \times\left[\frac{d_{0}^{t}\left(x_{0}^{t}, y_{0}^{t}\right)}{d_{0}^{t+1}\left(x_{0}^{t}, y_{0}^{t}\right)} \cdot \frac{d_{0}^{t}\left(x_{0}^{t+1}, y_{0}^{t+1}\right)}{d_{0}^{t+1}\left(x_{0}^{t+1}, y_{0}^{t+1}\right)}\right]^{\frac{1}{2}} \\
& =P E C_{0} \times P T C_{0},
\end{aligned}
$$

All pessimistic period measures are presented in Appendix 2.2. As shown in Appendix 3.2, the pessimistic Super-SBM model can also be applied for further assessment of anti-efficient $D M U s$, with $d_{0}(\cdot)=1$ [50]. In this regard, the pessimistic period measures, $\tilde{d}_{0}(\cdot) \leq 1$, are 
driven and illustrated in Appendix 4.2. In the situation where $d_{0}(\cdot)=1$, the corresponding $\tilde{d}_{0}(\cdot)$ is used.

Finally, the double-frontier MPI (represented by $\left.D F M P I_{0}\right)$ for evaluating the $D M U_{0}$ can be computed by the geometric mean of $O M P I_{0}$ and $P M P I_{0}$ as follows:

$$
\begin{aligned}
& D F M P I_{0}=\left[O M P I_{0} \times P M P I_{0}\right]^{\frac{1}{2}} \\
& =\left[D F E C_{0}\right]^{1 / 2} \times\left[D F T C_{0}\right]^{1 / 2}
\end{aligned}
$$

where $D F E C_{0}$ and $D F T C_{0}$ indicate the aggregated efficiency and technical changes over a time period.

$D F M P I_{0}>1$ and $D F M P I_{0}<1$ respectively demonstrate a progress and a regression over time that is more realistic. The productivity of $D M U_{0}$ will be unchanged if $D F M P I_{0}=1$.

\section{Road safety performance}

In this section, a three-year evaluation of Iranian road safety performance is practically carried out by means of a new hybrid approach, DF-SBM-ER. In this regard, the efficiencies obtained from the optimistic and pessimistic SBM models are aggregated by the ER algorithm, as a method of combination. Then, the efficiency and technical changes are examined. This case study includes 31 provinces.

The required data are usually selected based on data availability and safety programs defined by government and authorities. For example, Odeck (2000) considered vehicle technical failures as the main cause of road accidents. They defined these indicators mainly based on the Norwegian safety program [3]. Hermans et al. (2008) also utilized seven risk indicators presented in [51] as the input variables [5]. Shen et al. [8, 9] evaluated the road safety of European countries using a set of three input variables provided by the European Commission [52, 53]. Egilmez and McAvoy [10] assessed 50 U.S. states using an online database of Research and Innovative Technology Administration (RITA) Bureau of Transportation Statistics [54].

The Iran Road Maintenance and Transportation Organization (RMTO) is responsible for the intercity road safety performance. RMTO focuses more on fatality reduction by investing facilities and equipment. All available data are annually published by RMTO [55-57]. In this regard, six inputs and an output were derived from the Statistical Yearbook published annually by RMTO. Behnood et al. also applied RMTO Statistical Yearbook in order to define inputs and outputs variables [14].

The computations are based on the available data set for the years 2014-2016 obtained, including six inputs and one output as follows:

\subsection{Inputs}

\subsubsection{Police station (PS)}

The average number of highway police stations along 100 kilometres of road.

\subsubsection{Road maintenance depot (RMD)}

The average number of stations along 100 kilometres of road.

\subsubsection{Equipment and vehicles (E\&V)}

The average number of both equipment and vehicles along 100 kilometres of road.

\subsubsection{Camera (C)}

The average number of both fixed speed and monitoring cameras along 100 kilometres of road.

\begin{tabular}{|c|c|c|c|c|}
\hline Variables & Min & Max & Mean & SD \\
\hline \multicolumn{5}{|l|}{ a) Year $2014(\mathrm{~N}=31)$} \\
\hline \multicolumn{5}{|l|}{ Inputs } \\
\hline Police Station (PS) & 0.09 & 1.07 & 0.3620 & 0.21626 \\
\hline Road Maintenance Depot (RMD) & 0.14 & 3.47 & 1.1341 & 0.71446 \\
\hline Equipment \& Vehicles (E\&V) & 6.92 & 39.73 & 20.1556 & 8.03757 \\
\hline Camera (C) & 0.18 & 5.60 & 1.4881 & 1.20887 \\
\hline Emergency Medical Service (EMS) & 1.06 & 7.47 & 2.3653 & 1.35491 \\
\hline Road with Lighting System (RLS) & 0.92 & 29.81 & 7.5306 & 7.40396 \\
\hline \multicolumn{5}{|l|}{ Output } \\
\hline$F R^{-1}$ & 0.13 & 7.77 & 1.1632 & 1.37304 \\
\hline \multicolumn{5}{|l|}{ b) Year $2015(\mathrm{~N}=31)$} \\
\hline \multicolumn{5}{|l|}{ Inputs } \\
\hline Police Station (PS) & 0.11 & 1.02 & 0.3591 & 0.21112 \\
\hline Road Maintenance Depot (RMD) & 0.17 & 3.31 & 1.1205 & 0.69042 \\
\hline Equipment \& Vehicles (E\&V) & 9.21 & 37.91 & 20.4749 & 7.23863 \\
\hline Camera (C) & 0.42 & 6.93 & 1.8415 & 1.57520 \\
\hline Emergency Medical Service (EMS) & 1.20 & 7.63 & 2.4252 & 1.32906 \\
\hline Road with Lighting System (RLS) & 0.94 & 30.42 & 8.0667 & 7.81487 \\
\hline \multicolumn{5}{|l|}{ Output } \\
\hline$F R^{-1}$ & 0.16 & 8.03 & 1.1376 & 1.40518 \\
\hline \multicolumn{5}{|l|}{ c) Year $2016(\mathrm{~N}=31)$} \\
\hline \multicolumn{5}{|l|}{ Inputs } \\
\hline Police Station (PS) & 0.09 & 1.06 & 0.3586 & 0.21049 \\
\hline Road Maintenance Depot (RMD) & 0.18 & 3.17 & 1.1215 & 0.69748 \\
\hline Equipment \& Vehicles (E\&V) & 8.74 & 39.26 & 19.8316 & 7.20473 \\
\hline Camera (C) & 0.65 & 10.49 & 2.6766 & 2.45488 \\
\hline Emergency Medical Service (EMS) & 1.12 & 7.32 & 2.4191 & 1.31821 \\
\hline Road with Lighting System (RLS) & 1.27 & 31.91 & 8.2670 & 7.64173 \\
\hline \multicolumn{5}{|l|}{ Output } \\
\hline$F R^{-1}$ & 0.12 & 5.12 & 0.8052 & 0.87499 \\
\hline
\end{tabular}

Table 1 Descriptive statistics of data set 


\subsubsection{Emergency medical service (EMS)}

The average number of EMS stations along 100 kilometres of road.

\subsubsection{Road with lighting system (RLS)}

The average length of road equipped with lighting systems along 100 kilometres of road.

\subsection{Output}

\subsubsection{Fatality risk $\left(F R^{-1}\right)$}

The inverse of fatality risk, including the number of fatalities per mean rate of hourly traffic.
It is supposed that all police stations are similar in terms of the number of officers and patrols. It is also supposed that all other input variables are the same. The period of 2014-2016 was selected due to data availability for the inputs and the output considered. The descriptive statistics of data are reported in Table 1. Table 2 reveals that the selected data set for the years 2014-2016 are highly correlated. Tables 3, 4 and 5 depict the efficiency and anti-efficiency degrees measured by implementing the optimistic and pessimistic SBM models (3) and (6). According to the results reported in column 2 of Table 3, Fars is optimistically recognized as an efficient province along with Alborz, Ilam, Tehran, Khorasan S,

Table 2 Correlation matrix of all variables

\begin{tabular}{|c|c|c|c|c|c|c|c|}
\hline Variables & $\begin{array}{l}\text { Police } \\
\text { Station (PS) }\end{array}$ & $\begin{array}{l}\text { Road Maintenance } \\
\text { Depot (RMD) }\end{array}$ & $\begin{array}{l}\text { Equipment \& } \\
\text { Vehicles (E\&V) }\end{array}$ & $\begin{array}{l}\text { Camera } \\
\text { (C) }\end{array}$ & $\begin{array}{l}\text { Emergency Medical } \\
\text { Service (EMS) }\end{array}$ & $\begin{array}{l}\text { Road with Lighting } \\
\text { System (RLS) }\end{array}$ & $F R^{-1}$ \\
\hline \multicolumn{8}{|l|}{ a) 2014} \\
\hline Police Station (PS) & 1 & $0.830^{* *}$ & $0.758^{* *}$ & $0.727^{* *}$ & $0.923^{* *}$ & $0.858^{* *}$ & $0.798^{* *}$ \\
\hline $\begin{array}{l}\text { Road Maintenance } \\
\text { Depot (RMD) }\end{array}$ & & 1 & $0.788^{* *}$ & $0.643^{* *}$ & $0.797^{* *}$ & $0.638^{* *}$ & $0.722^{* *}$ \\
\hline $\begin{array}{l}\text { Equipment \& Vehicles } \\
(\mathrm{E} \& \mathrm{~V})\end{array}$ & & & 1 & $0.659^{* *}$ & $0.658^{* *}$ & $0.619^{* *}$ & $0.571^{* *}$ \\
\hline Camera (C) & & & & 1 & $0.659^{* *}$ & $0.736^{* *}$ & $0.718^{* *}$ \\
\hline $\begin{array}{l}\text { Emergency Medical } \\
\text { Service (EMS) }\end{array}$ & & & & & 1 & $0.798^{* *}$ & $0.838^{* *}$ \\
\hline $\begin{array}{l}\text { Road with Lighting } \\
\text { System (RLS) }\end{array}$ & & & & & & 1 & $0.733^{* *}$ \\
\hline$F R^{-1}$ & & & & & & & 1 \\
\hline \multicolumn{8}{|l|}{ b) 2015} \\
\hline Police Station (PS) & 1 & $0.821^{* *}$ & $0.764^{* *}$ & $0.796^{* *}$ & $0.914^{* *}$ & $0.863^{* *}$ & $0.809 * *$ \\
\hline $\begin{array}{l}\text { Road Maintenance } \\
\text { Depot (RMD) }\end{array}$ & & 1 & $0.790^{* *}$ & $0.578^{* *}$ & $0.795^{* *}$ & $0.647^{* *}$ & $0.766^{* *}$ \\
\hline $\begin{array}{l}\text { Equipment \& Vehicles } \\
(E \& V)\end{array}$ & & & 1 & $0.573^{* *}$ & $0.676^{* *}$ & $0.651^{* *}$ & $0.626^{* *}$ \\
\hline Camera (C) & & & & 1 & $0.673^{* *}$ & $0.860^{* *}$ & $0.670^{* *}$ \\
\hline $\begin{array}{l}\text { Emergency Medical } \\
\text { Service (EMS) }\end{array}$ & & & & & 1 & $0.777^{* *}$ & $0.887^{* *}$ \\
\hline $\begin{array}{l}\text { Road with Lighting } \\
\text { System (RLS) }\end{array}$ & & & & & & 1 & $0.702^{* *}$ \\
\hline$F R^{-1}$ & & & & & & & 1 \\
\hline \multicolumn{8}{|l|}{ c) 2016} \\
\hline Police Station (PS) & 1 & $0.814^{* *}$ & $0.796^{* *}$ & $0.895^{* *}$ & $0.916^{* *}$ & $0.867^{* *}$ & $0.663^{* *}$ \\
\hline $\begin{array}{l}\text { Road Maintenance } \\
\text { Depot (RMD) }\end{array}$ & & 1 & $0.791^{* *}$ & $0.695^{* *}$ & $0.789 * *$ & $0.646^{* *}$ & $0.653^{* *}$ \\
\hline $\begin{array}{l}\text { Equipment \& Vehicles } \\
(E \& V)\end{array}$ & & & 1 & $0.559^{* *}$ & $0.655^{* *}$ & $0.660^{* *}$ & $0.394^{*}$ \\
\hline Camera (C) & & & & 1 & $0.837^{* *}$ & $0.866^{* *}$ & $0.696^{* *}$ \\
\hline $\begin{array}{l}\text { Emergency Medical } \\
\text { Service (EMS) }\end{array}$ & & & & & 1 & $0.788^{* *}$ & $0.744^{* *}$ \\
\hline $\begin{array}{l}\text { Road with Lighting } \\
\text { System (RLS) }\end{array}$ & & & & & & 1 & $0.585^{* *}$ \\
\hline$F R^{-1}$ & & & & & & & 1 \\
\hline
\end{tabular}

**Correlation is significant at the 0.01 level (2-tailed).

*Correlation is significant at the 0.05 level (2-tailed). 
Table 3 Efficiency results for the 31 Iranian provinces in terms of road safety performance for the year 2014

\begin{tabular}{|c|c|c|c|c|c|c|c|c|c|c|c|}
\hline \multirow[t]{3}{*}{ Province } & \multicolumn{4}{|c|}{ Optimistic SBM } & \multicolumn{4}{|c|}{ Pessimistic SBM } & \multirow{2}{*}{\multicolumn{2}{|c|}{$\frac{\text { Safety performance }}{\text { Assessment distribution }}$}} & \multirow[b]{3}{*}{ Rank } \\
\hline & \multirow[b]{2}{*}{$T_{\text {optimistic }}$} & \multirow[b]{2}{*}{ Rank } & \multicolumn{2}{|c|}{ Assessment distribution } & \multirow[b]{2}{*}{$\tau_{\text {pessimistic }}$} & \multirow[b]{2}{*}{ Rank } & \multicolumn{2}{|c|}{ Assessment distribution } & & & \\
\hline & & & $H_{1}$ & $\mathrm{H}_{2}$ & & & $H_{1}$ & $\mathrm{H}_{2}$ & $H_{1}$ & $\mathrm{H}_{2}$ & \\
\hline Azerbaijan E & 0.2828 & 24 & 0.7172 & 0.2828 & 0.4346 & 24 & 0.4346 & 0.5654 & 0.5921 & 0.4079 & 24 \\
\hline Azerbaijan W & 0.3427 & 21 & 0.6573 & 0.3427 & 0.3506 & 21 & 0.3506 & 0.6494 & 0.5048 & 0.4952 & 21 \\
\hline Ardabil & 0.2414 & 27 & 0.7586 & 0.2414 & 1.0000 & 29 & 1.0000 & 0.0000 & 0.9125 & 0.0875 & 29 \\
\hline Isfahan & 0.3308 & 22 & 0.6692 & 0.3308 & 0.3659 & 22 & 0.3659 & 0.6341 & 0.5211 & 0.4789 & 22 \\
\hline Alborz & 1.0000 & 1 & 0.0000 & 1.0000 & 0.1093 & 1 & 0.1093 & 0.8907 & 0.0378 & 0.9622 & 1 \\
\hline Ilam & 1.0000 & 1 & 0.0000 & 1.0000 & 0.2083 & 9 & 0.2083 & 0.7917 & 0.0746 & 0.9254 & 7 \\
\hline Bushehr & 0.4626 & 17 & 0.5374 & 0.4626 & 0.2500 & 15 & 0.2500 & 0.7500 & 0.3715 & 0.6285 & 16 \\
\hline Tehran & 1.0000 & 1 & 0.0000 & 1.0000 & 0.1832 & 8 & 0.1832 & 0.8168 & 0.0650 & 0.9350 & 6 \\
\hline Chaharmahal and Bakhtiari & 0.6986 & 9 & 0.3014 & 0.6986 & 0.2250 & 12 & 0.2250 & 0.7750 & 0.2277 & 0.7723 & 11 \\
\hline Khorasan S & 1.0000 & 1 & 0.0000 & 1.0000 & 0.1640 & 5 & 0.1640 & 0.8360 & 0.0578 & 0.9422 & 4 \\
\hline Khorasan R & 0.2126 & 29 & 0.7874 & 0.2126 & 0.5686 & 28 & 0.5686 & 0.4314 & 0.7103 & 0.2897 & 28 \\
\hline Khorasan N & 0.6563 & 11 & 0.3437 & 0.6563 & 0.1787 & 6 & 0.1787 & 0.8213 & 0.2245 & 0.7755 & 10 \\
\hline Khuzestan & 1.0000 & 1 & 0.0000 & 1.0000 & 0.1816 & 7 & 0.1816 & 0.8184 & 0.0644 & 0.9356 & 5 \\
\hline Zanjan & 0.2332 & 28 & 0.7668 & 0.2332 & 0.5059 & 27 & 0.5059 & 0.4941 & 0.6634 & 0.3366 & 27 \\
\hline Semnan & 0.4515 & 18 & 0.5485 & 0.4515 & 0.2536 & 16 & 0.2536 & 0.7464 & 0.3801 & 0.6199 & 17 \\
\hline Sistan and Baluchistan & 0.1287 & 31 & 0.8713 & 0.1287 & 1.0000 & 29 & 1.0000 & 0.0000 & 0.9552 & 0.0448 & 31 \\
\hline Fars & 1.0000 & 1 & 0.0000 & 1.0000 & 0.2540 & 17 & 0.2540 & 0.7460 & 0.0925 & 0.9075 & 8 \\
\hline Qazvin & 1.0000 & 1 & 0.0000 & 1.0000 & 0.1238 & 3 & 0.1238 & 0.8762 & 0.0430 & 0.9570 & 3 \\
\hline Qom & 0.5263 & 12 & 0.4737 & 0.5263 & 0.2118 & 10 & 0.2118 & 0.7882 & 0.3124 & 0.6876 & 12 \\
\hline Kurdistan & 0.2649 & 25 & 0.7351 & 0.2649 & 0.4707 & 25 & 0.4707 & 0.5293 & 0.6242 & 0.3758 & 25 \\
\hline Kerman & 0.2856 & 23 & 0.7144 & 0.2856 & 0.4260 & 23 & 0.4260 & 0.5740 & 0.5853 & 0.4147 & 23 \\
\hline Kermamshah & 0.3804 & 20 & 0.6196 & 0.3804 & 0.2866 & 20 & 0.2866 & 0.7134 & 0.4425 & 0.5575 & 20 \\
\hline Kohgiluyeh and Boyer-Ahmad & 0.4649 & 15 & 0.5351 & 0.4649 & 0.2686 & 18 & 0.2686 & 0.7314 & 0.3814 & 0.6186 & 18 \\
\hline Golestan & 0.4712 & 14 & 0.5288 & 0.4712 & 0.2330 & 14 & 0.2330 & 0.7670 & 0.3562 & 0.6438 & 14 \\
\hline Guilan & 0.3988 & 19 & 0.6012 & 0.3988 & 0.2752 & 19 & 0.2752 & 0.7248 & 0.4245 & 0.5755 & 19 \\
\hline Lorestan & 0.1545 & 30 & 0.8455 & 0.1545 & 1.0000 & 29 & 1.0000 & 0.0000 & 0.9457 & 0.0543 & 30 \\
\hline Mazandaran & 0.5023 & 13 & 0.4977 & 0.5023 & 0.2171 & 11 & 0.2171 & 0.7829 & 0.3290 & 0.6710 & 13 \\
\hline Markazi & 0.4632 & 16 & 0.5368 & 0.4632 & 0.2301 & 13 & 0.2301 & 0.7699 & 0.3590 & 0.6410 & 15 \\
\hline Hormozgan & 1.0000 & 1 & 0.0000 & 1.0000 & 0.1199 & 2 & 0.1199 & 0.8801 & 0.0416 & 0.9584 & 2 \\
\hline Hamedan & 0.2456 & 26 & 0.7544 & 0.2456 & 0.4794 & 26 & 0.4794 & 0.5206 & 0.6409 & 0.3591 & 26 \\
\hline Yazd & 0.6968 & 10 & 0.3032 & 0.6968 & 0.1633 & 4 & 0.1633 & 0.8367 & 0.1960 & 0.8040 & 9 \\
\hline
\end{tabular}

Khuzestan, Qazvin and Hormozgan, and it is ranked $17^{\text {th }}$, with an efficiency degree of 0.7460 from the pessimistic point of view, as shown in column 6 of Table 3. Obviously, the optimistic SBM model usually overestimates the efficiencies of Iranian provinces, since about 25 per cent of provinces are recognized as efficient according to the data set belonging to 2014. Consequently, the efficiency results might be biased. Furthermore, both efficiency and anti-efficiency degrees are important for having a better insight into the situation of each province in terms of road safety performance. For example, although Kermanshah ranks $20^{\text {th }}$ from both optimistic and pessimistic points of view, the measured efficiency scores are respectively 0.3804 and 0.7134 , which may lead to different policies. For this reason, the simultaneous evaluations of road safety performance based on the two perspectives seem to be necessary.

A comprehensive and unique indicator of road safety performance can be obtained by employing the ER algorithm as a method of combination. As presented in 
Table 4 Efficiency results for the 31 Iranian provinces in terms of road safety performance for the year 2015

\begin{tabular}{|c|c|c|c|c|c|c|c|c|c|c|c|}
\hline \multirow[t]{3}{*}{ Province } & \multicolumn{4}{|c|}{ Optimistic SBM } & \multicolumn{4}{|c|}{ Pessimistic SBM } & \multicolumn{3}{|c|}{ Safety performance } \\
\hline & \multirow[b]{2}{*}{$T_{\text {optimistic }}$} & \multirow[b]{2}{*}{ Rank } & \multicolumn{2}{|c|}{ Assessment distribution } & \multirow[b]{2}{*}{$T_{\text {pessimistic }}$} & \multirow[b]{2}{*}{ Rank } & \multicolumn{2}{|c|}{ Assessment distribution } & \multicolumn{2}{|c|}{ Assessment distribution } & \multirow[b]{2}{*}{ Rank } \\
\hline & & & $H_{1}$ & $\mathrm{H}_{2}$ & & & $H_{1}$ & $\mathrm{H}_{2}$ & $H_{1}$ & $\mathrm{H}_{2}$ & \\
\hline Azerbaijan E & 0.2863 & 23 & 0.7137 & 0.2863 & 0.5547 & 22 & 0.5547 & 0.4453 & 0.6595 & 0.3405 & 21 \\
\hline Azerbaijan W & 0.2927 & 22 & 0.7073 & 0.2927 & 0.5420 & 20 & 0.5420 & 0.4580 & 0.6485 & 0.3515 & 20 \\
\hline Ardabil & 0.3970 & 16 & 0.6030 & 0.3970 & 0.3788 & 15 & 0.3788 & 0.6212 & 0.4890 & 0.5110 & 15 \\
\hline Isfahan & 0.2740 & 25 & 0.7260 & 0.2740 & 0.6164 & 23 & 0.6164 & 0.3836 & 0.7012 & 0.2988 & 23 \\
\hline Alborz & 1.0000 & 1 & 0.0000 & 1.0000 & 0.1438 & 1 & 0.1438 & 0.8562 & 0.0503 & 0.9497 & 1 \\
\hline Ilam & 1.0000 & 1 & 0.0000 & 1.0000 & 0.2667 & 4 & 0.2667 & 0.7333 & 0.0976 & 0.9024 & 3 \\
\hline Bushehr & 0.5171 & 8 & 0.4829 & 0.5171 & 0.2917 & 7 & 0.2917 & 0.7083 & 0.3651 & 0.6349 & 8 \\
\hline Tehran & 0.4281 & 12 & 0.5719 & 0.4281 & 0.3260 & 11 & 0.3260 & 0.6740 & 0.4381 & 0.5619 & 12 \\
\hline Chaharmahal and Bakhtiari & 1.0000 & 1 & 0.0000 & 1.0000 & 0.2880 & 6 & 0.2880 & 0.7120 & 0.1062 & 0.8938 & 5 \\
\hline Khorasan S & 0.5001 & 9 & 0.4999 & 0.5001 & 0.3232 & 10 & 0.3232 & 0.6768 & 0.3939 & 0.6061 & 9 \\
\hline Khorasan R & 0.1673 & 30 & 0.8327 & 0.1673 & 1.0000 & 25 & 1.0000 & 0.0000 & 0.9409 & 0.0591 & 30 \\
\hline Khorasan N & 0.4571 & 11 & 0.5429 & 0.4571 & 0.3426 & 12 & 0.3426 & 0.6574 & 0.4309 & 0.5691 & 11 \\
\hline Khuzestan & 1.0000 & 1 & 0.0000 & 1.0000 & 0.2938 & 8 & 0.2938 & 0.7062 & 0.1086 & 0.8914 & 6 \\
\hline Zanjan & 0.3010 & 21 & 0.6990 & 0.3010 & 0.5300 & 19 & 0.5300 & 0.4700 & 0.6367 & 0.3633 & 19 \\
\hline Semnan & 0.3173 & 18 & 0.6827 & 0.3173 & 0.4899 & 17 & 0.4899 & 0.5101 & 0.6037 & 0.3963 & 17 \\
\hline Sistan and Baluchistan & 0.2136 & 28 & 0.7864 & 0.2136 & 1.0000 & 25 & 1.0000 & 0.0000 & 0.9233 & 0.0767 & 28 \\
\hline Fars & 0.2824 & 24 & 0.7176 & 0.2824 & 0.5508 & 21 & 0.5508 & 0.4492 & 0.6596 & 0.3404 & 22 \\
\hline Qazvin & 0.4634 & 10 & 0.5366 & 0.4634 & 0.3172 & 9 & 0.3172 & 0.6828 & 0.4118 & 0.5882 & 10 \\
\hline Qom & 0.4203 & 14 & 0.5797 & 0.4203 & 1.0000 & 25 & 1.0000 & 0.0000 & 0.8371 & 0.1629 & 25 \\
\hline Kurdistan & 0.2615 & 26 & 0.7385 & 0.2615 & 1.0000 & 25 & 1.0000 & 0.0000 & 0.9045 & 0.0955 & 27 \\
\hline Kerman & 0.1914 & 29 & 0.8086 & 0.1914 & 1.0000 & 25 & 1.0000 & 0.0000 & 0.9319 & 0.0681 & 29 \\
\hline Kermamshah & 0.4265 & 13 & 0.5735 & 0.4265 & 0.3623 & 14 & 0.3623 & 0.6377 & 0.4612 & 0.5388 & 13 \\
\hline Kohgiluyeh and Boyer-Ahmad & 1.0000 & 1 & 0.0000 & 1.0000 & 0.2386 & 2 & 0.2386 & 0.7614 & 0.0864 & 0.9136 & 2 \\
\hline Golestan & 0.4128 & 15 & 0.5872 & 0.4128 & 0.3534 & 13 & 0.3534 & 0.6466 & 0.4640 & 0.5360 & 14 \\
\hline Guilan & 0.3162 & 19 & 0.6838 & 0.3162 & 0.4900 & 18 & 0.4900 & 0.5100 & 0.6044 & 0.3956 & 18 \\
\hline Lorestan & 0.1672 & 31 & 0.8328 & 0.1672 & 1.0000 & 25 & 1.0000 & 0.0000 & 0.9410 & 0.0590 & 31 \\
\hline Mazandaran & 0.3851 & 17 & 0.6149 & 0.3851 & 0.3989 & 16 & 0.3989 & 0.6011 & 0.5084 & 0.4916 & 16 \\
\hline Markazi & 0.3026 & 20 & 0.6974 & 0.3026 & 1.0000 & 25 & 1.0000 & 0.0000 & 0.8878 & 0.1122 & 26 \\
\hline Hormozgan & 1.0000 & 1 & 0.0000 & 1.0000 & 0.2685 & 5 & 0.2685 & 0.7315 & 0.0983 & 0.9017 & 4 \\
\hline Hamedan & 0.2295 & 27 & 0.7705 & 0.2295 & 0.7268 & 24 & 0.7268 & 0.2732 & 0.7844 & 0.2156 & 24 \\
\hline Yazd & 0.6286 & 7 & 0.3714 & 0.6286 & 0.2410 & 3 & 0.2410 & 0.7590 & 0.2735 & 0.7265 & 7 \\
\hline
\end{tabular}

section 3, a frame of discernment, $\Theta=\left\{H_{1}, H_{2}\right\}$, consisting of two hypotheses, $H_{1}$ : not-efficient and $H_{2}$ : efficient, is first defined $[18,19]$. There are also two pieces of evidence, including the pessimistic and optimistic efficiencies. Accordingly, $E_{j}=\left\{e_{j 1}, e_{j 2}\right\}$ can be defined as follows:

$$
\begin{aligned}
& S\left(e_{j 1}\right)=\left\{\left(H_{1}, \beta_{j, 1,1}\right),\left(H_{2}, \beta_{j, 2,1}\right)\right\}, j=1, \ldots, n \\
& S\left(e_{j 2}\right)=\left\{\left(H_{1}, \beta_{j, 1,2}\right),\left(H_{2}, \beta_{j, 2,2}\right)\right\}, j=1, \ldots, n
\end{aligned}
$$

where $S\left(e_{j 1}\right)$ and $S\left(e_{j 2}\right)$ respectively represent two assessment distributions regarding $D M U_{0}$, taking into account the optimistic and pessimistic perspectives. $\left(\beta_{j, 1,1}\right.$, $\left.\beta_{j, 2,1}\right)$ and $\left(\beta_{j, 1,2}, \beta_{j, 2,2}\right)$ respectively denote the belief degrees assigned to the propositions $H_{1}$ and $H_{2}$ based on the SBM models (3) and (6). Obviously, $\beta_{j, 1,1}$ $+\beta_{j, 2,1}=1$ and $\beta_{j, 1,2}+\beta_{j, 2,2}=1$. Subsequently, the assessment distributions (28) and (29) are transformed to the following mass functions:

$$
\begin{aligned}
& m_{j, 1,1}=\omega_{1} \beta_{j, 1,1}, m_{j, 2,1}=\omega_{1} \beta_{j, 2,1}, m_{j, \theta, 1}=1-\left(m_{j, 1,1}+m_{j, 2,1}\right), \\
& \tilde{m}_{j, \theta, 1}=\omega_{1}\left(1-\beta_{j, 1,1}-\beta_{j, 2,1} j=1, \ldots, n\right)
\end{aligned}
$$


Table 5 Efficiency results for the 31 Iranian provinces in terms of road safety performance for the year 2016

\begin{tabular}{|c|c|c|c|c|c|c|c|c|c|c|c|}
\hline \multirow[t]{3}{*}{ Province } & \multicolumn{4}{|c|}{ Optimistic SBM } & \multicolumn{4}{|c|}{ Pessimistic SBM } & \multicolumn{3}{|c|}{ Safety performance } \\
\hline & \multirow[b]{2}{*}{$T_{\text {optimistic }}$} & \multirow[b]{2}{*}{ Rank } & \multicolumn{2}{|c|}{ Assessment distribution } & \multirow[b]{2}{*}{$T_{\text {pessimistic }}$} & \multirow[b]{2}{*}{ Rank } & \multicolumn{2}{|c|}{ Assessment distribution } & \multicolumn{2}{|c|}{ Assessment distribution } & \multirow[b]{2}{*}{ Rank } \\
\hline & & & $H_{1}$ & $\mathrm{H}_{2}$ & & & $H_{1}$ & $\mathrm{H}_{2}$ & $H_{1}$ & $\mathrm{H}_{2}$ & \\
\hline Azerbaijan E & 0.3343 & 21 & 0.6657 & 0.3343 & 0.4984 & 21 & 0.4984 & 0.5016 & 0.5985 & 0.4015 & 20 \\
\hline Azerbaijan W & 0.2812 & 25 & 0.7188 & 0.2812 & 0.5939 & 24 & 0.5939 & 0.4061 & 0.6846 & 0.3154 & 24 \\
\hline Ardabil & 0.4724 & 16 & 0.5276 & 0.4724 & 0.3188 & 15 & 0.3188 & 0.6812 & 0.4075 & 0.5925 & 16 \\
\hline Isfahan & 0.2353 & 27 & 0.7647 & 0.2353 & 0.6655 & 26 & 0.6655 & 0.3345 & 0.7494 & 0.2506 & 26 \\
\hline Alborz & 1.0000 & 1 & 0.0000 & 1.0000 & 0.1403 & 2 & 0.1403 & 0.8597 & 0.0491 & 0.9509 & 2 \\
\hline Ilam & 1.0000 & 1 & 0.0000 & 1.0000 & 0.1297 & 1 & 0.1297 & 0.8703 & 0.0452 & 0.9548 & 1 \\
\hline Bushehr & 0.5236 & 11 & 0.4764 & 0.5236 & 0.2935 & 11 & 0.2935 & 0.7065 & 0.3625 & 0.6375 & 10 \\
\hline Tehran & 0.1575 & 31 & 0.8425 & 0.1575 & 1.0000 & 27 & 1.0000 & 0.0000 & 0.9446 & 0.0554 & 31 \\
\hline Chaharmahal and Bakhtiari & 0.6007 & 8 & 0.3993 & 0.6007 & 0.2593 & 7 & 0.2593 & 0.7407 & 0.2991 & 0.7009 & 7 \\
\hline Khorasan S & 0.6784 & 5 & 0.3216 & 0.6784 & 0.2279 & 6 & 0.2279 & 0.7721 & 0.2398 & 0.7602 & 5 \\
\hline Khorasan R & 0.2047 & 29 & 0.7953 & 0.2047 & 1.0000 & 27 & 1.0000 & 0.0000 & 0.9268 & 0.0732 & 29 \\
\hline Khorasan N & 0.5569 & 9 & 0.4431 & 0.5569 & 0.2751 & 8 & 0.2751 & 0.7249 & 0.3326 & 0.6674 & 9 \\
\hline Khuzestan & 0.4288 & 18 & 0.5712 & 0.4288 & 0.3861 & 18 & 0.3861 & 0.6139 & 0.4742 & 0.5258 & 18 \\
\hline Zanjan & 0.3922 & 19 & 0.6078 & 0.3922 & 0.3920 & 19 & 0.3920 & 0.6080 & 0.4999 & 0.5001 & 19 \\
\hline Semnan & 0.4869 & 15 & 0.5131 & 0.4869 & 0.3207 & 16 & 0.3207 & 0.6793 & 0.4001 & 0.5999 & 15 \\
\hline Sistan and Baluchistan & 0.2079 & 28 & 0.7921 & 0.2079 & 1.0000 & 27 & 1.0000 & 0.0000 & 0.9255 & 0.0745 & 28 \\
\hline Fars & 0.2984 & 24 & 0.7016 & 0.2984 & 0.5086 & 22 & 0.5086 & 0.4914 & 0.6259 & 0.3741 & 22 \\
\hline Qazvin & 0.6651 & 6 & 0.3349 & 0.6651 & 0.2275 & 5 & 0.2275 & 0.7725 & 0.2466 & 0.7534 & 6 \\
\hline Qom & 0.5034 & 14 & 0.4966 & 0.5034 & 0.2778 & 9 & 0.2778 & 0.7222 & 0.3647 & 0.6353 & 11 \\
\hline Kurdistan & 0.3895 & 20 & 0.6105 & 0.3895 & 1.0000 & 27 & 1.0000 & 0.0000 & 0.8508 & 0.1492 & 27 \\
\hline Kerman & 0.2612 & 26 & 0.7388 & 0.2612 & 0.6127 & 25 & 0.6127 & 0.3873 & 0.7065 & 0.2935 & 25 \\
\hline Kermamshah & 0.5306 & 10 & 0.4694 & 0.5306 & 0.3082 & 13 & 0.3082 & 0.6918 & 0.3672 & 0.6328 & 12 \\
\hline Kohgiluyeh and Boyer-Ahmad & 1.0000 & 1 & 0.0000 & 1.0000 & 0.1602 & 3 & 0.1602 & 0.8398 & 0.0564 & 0.9436 & 3 \\
\hline Golestan & 0.6022 & 7 & 0.3978 & 0.6022 & 0.2835 & 10 & 0.2835 & 0.7165 & 0.3121 & 0.6879 & 8 \\
\hline Guilan & 0.4472 & 17 & 0.5528 & 0.4472 & 0.3637 & 17 & 0.3637 & 0.6363 & 0.4496 & 0.5504 & 17 \\
\hline Lorestan & 0.1849 & 30 & 0.8151 & 0.1849 & 1.0000 & 27 & 1.0000 & 0.0000 & 0.9343 & 0.0657 & 30 \\
\hline Mazandaran & 0.5226 & 13 & 0.4774 & 0.5226 & 0.3085 & 14 & 0.3085 & 0.6915 & 0.3720 & 0.6280 & 14 \\
\hline Markazi & 0.3209 & 22 & 0.6791 & 0.3209 & 0.4957 & 20 & 0.4957 & 0.5043 & 0.6049 & 0.3951 & 21 \\
\hline Hormozgan & 1.0000 & 1 & 0.0000 & 1.0000 & 0.1880 & 4 & 0.1880 & 0.8120 & 0.0669 & 0.9331 & 4 \\
\hline Hamedan & 0.3021 & 23 & 0.6979 & 0.3021 & 0.5386 & 23 & 0.5386 & 0.4614 & 0.6410 & 0.3590 & 23 \\
\hline Yazd & 0.5235 & 12 & 0.4765 & 0.5235 & 0.3038 & 12 & 0.3038 & 0.6962 & 0.3687 & 0.6313 & 13 \\
\hline
\end{tabular}

$$
\begin{aligned}
& m_{j, 1,2}=\omega_{2} \beta_{j, 1,2}, m_{j, 2,2}=\omega_{2} \beta_{j, 2,2}, m_{j, \theta, 2}=1-\left(m_{j, 1,2}+m_{j, 2,2}\right), \\
& \tilde{m}_{j, \theta, 2}=\omega_{2}\left(1-\beta_{j, 1,2}-\beta_{j, 2,2}\right) j=1, \ldots, n
\end{aligned}
$$

Eventually, the aggregated distributions can be computed using Eqs. (18)-(21). The final efficiency scores (column 11) and associated ranking results (column 12) for the years 2014-2016 are respectively reported in Tables 3, 4 and 5. Obviously, the discrimination power of DF-SBM-ER is higher than both the optimistic and pessimistic SBM models.

\section{Results and discussion}

As graphically shown in Figs. 1, 2 and 3, Alborz, Hormozgan, and Qazvin rank $1^{\text {st }}(0.9622), 2^{\text {nd }}(0.9584)$ and $3^{\text {rd }}(0.9570)$ in 2014 respectively; Alborz, Kohgiluyeh-and-Boyer-Ahmad and Ilam rank $1^{\text {st }}(0.9497), 2^{\text {nd }}(0.9136)$ and $3^{\text {rd }}(0.9024)$ in 2015 respectively; Ilam, Alborz and Kohgiluyeh-and-Boyer-Ahmad rank $1^{\text {st }}(0.9548), 2^{\text {nd }}(0.9509)$ and $3^{\text {rd }}(0.9436)$ in 2016 respectively.

Similar results can be obtained using the double frontier CCR model aggregated by ER approach (DF-CCR-ER). These results are compared in Table 6 and Fig. 4. For more discussion on DF-CCR-ER, the 


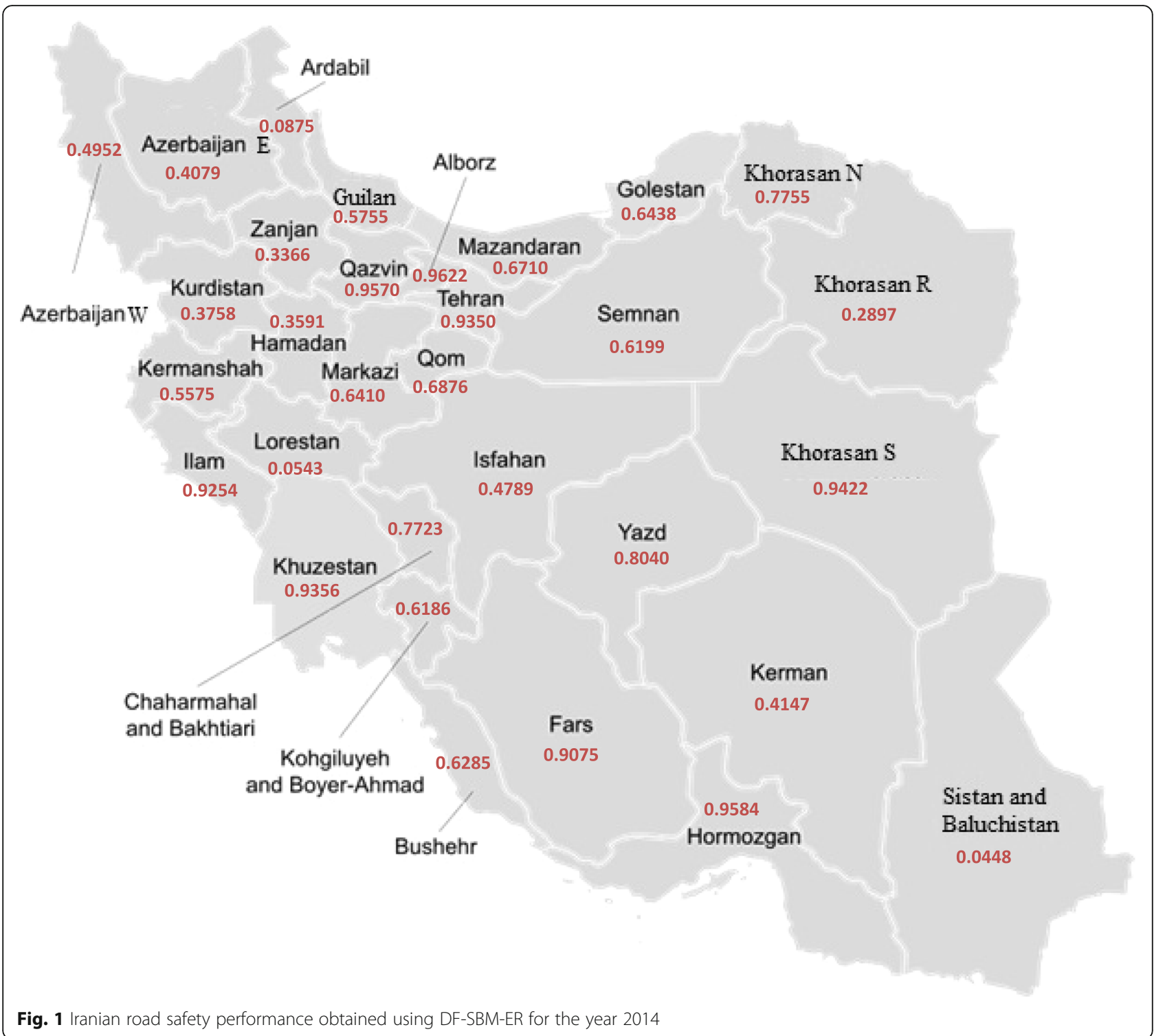

interested readers are referred to $[15,19]$. As proved, the efficiency and anti-efficiency degrees obtained by SBM are equal or less than those obtained by the CCR model. Compared with CCR, the SBM based models result in higher optimistic and lower pessimistic degrees of efficiency. As a result, some aggregated results achieved by DF-SBM-ER are greater than those obtained by DF-CCR-ER [15]. In such a situation, the aggregated efficiencies are closer to the pessimistic efficiency compared to the optimistic results.

The efficiency and anti-efficiency results obtained by SBM and Super SBM for evaluating 31 Iranian provinces over two periods of time (2014-2015 and 2015-2016) are summarized in Tables 7 and 8. In order to further analyzethe road safety performance over a period of time (2014-2016), the super efficiency scores of the efficient provinces, $D_{0}^{t}\left(x_{0}^{t}, y_{0}^{t}\right)=1$, are computed by implementing the optimistic Super SBM model (43), $\tilde{D}_{0}^{t}\left(x_{0}^{t}, y_{0}^{t}\right)$. For example, the super efficiency values of the eight efficient provinces in 2014, Alborz, Ilam, Tehran, Khorasan S, Khuzestan, Fars, Qazvin and Hormozgan, were $1.2176,1.0467,1.0349,1.0307,1.0125,1.0433$, 1.0269 and 1.2585 respectively. Similarly, the super anti-efficiency scores of anti-efficient provinces, $d_{0}^{t}\left(x_{0}^{t}\right.$, $\left.y_{0}^{t}\right)=1$, are computed by employing the pessimistic Super-SBM model (47), $\tilde{d}_{0}^{t}\left(x_{0}^{t}, y_{0}^{t}\right)$.

As shown in Table 7, twelve provinces optimistically improved their efficiency from 2014 to 2015 , as $O E C \geq 1$, while only three provinces experienced efficiency progress from the pessimistic point of view, as $P E C \geq 1$. Taking into account the optimistic point of view, 


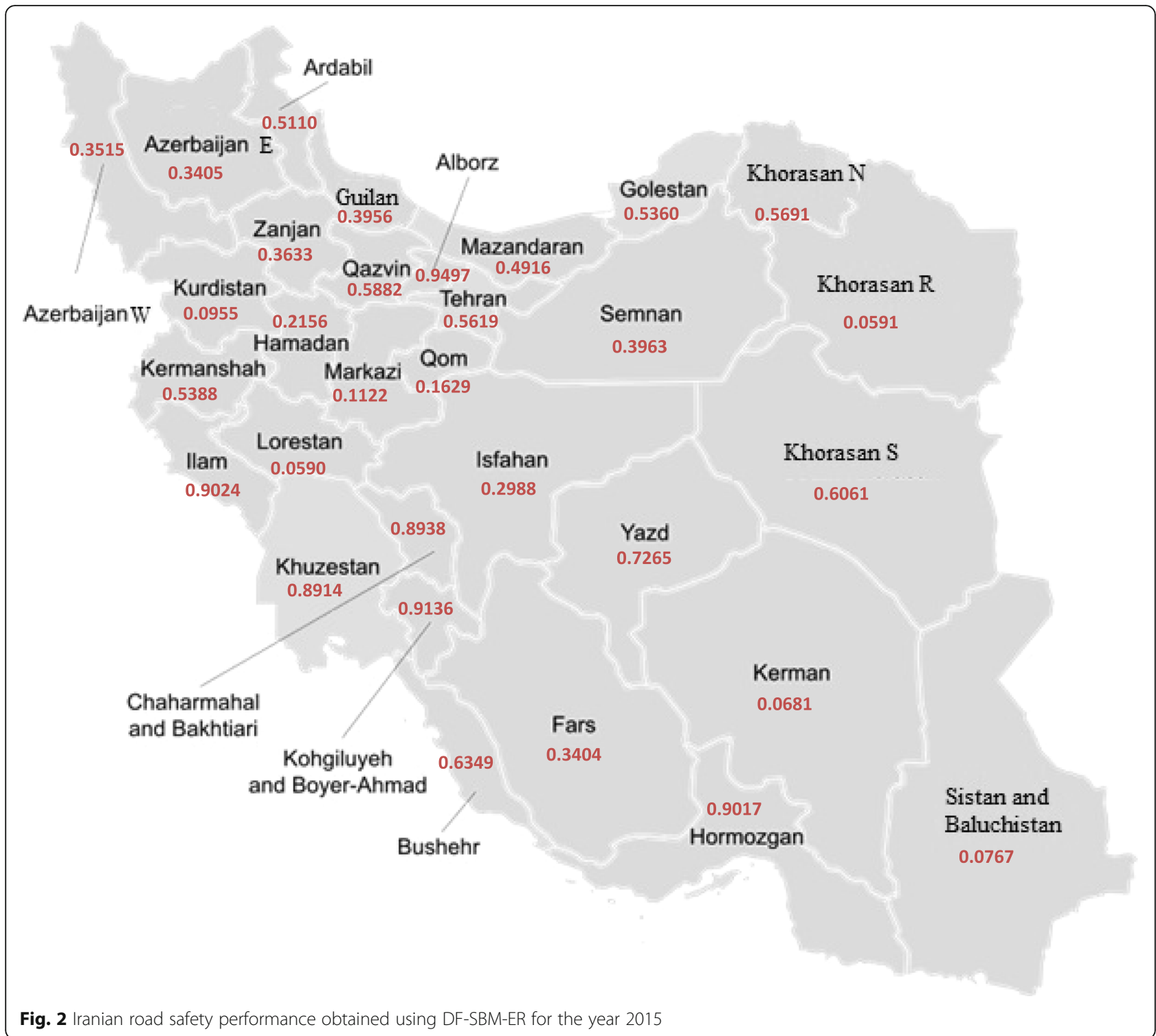

Kohgiluye-and-Boyer-Ahmad showed the highest efficiency progress, with a growth rate of $83.84 \%$ from 2014 to 2015, while Fars experienced the highest efficiency regression, with a decline of $72.93 \%$. On average, the Iranian road safety performance declined in terms of efficiency change from both the optimistic and pessimistic perspectives, with a rate of $11.61 \%$ and $37.27 \%$ decline respectively, from 2014-2015. Furthermore, among the twenty two provinces with optimistic technical progress, Mazandaran experienced the highest change, with an improvement of $5.58 \%$; meanwhile, Qom pessimistically experienced the highest technical change, with a growth rate of $127.83 \%$. It is noted that on average, the technical efficiency of provinces in terms of road safety performance optimistically declined by $2.43 \%$ while pessimistically improved $37.79 \%$. Fig. 5 (a) illustrates that the road safety performance of ten provinces optimistically progressed from 2014 to 2015, as $O M P I \geq 1$; additionally, nine provinces pessimistically showed improvement in terms of road safety performance, as $P M P I \geq 1$. Generally speaking, the Iranian road safety performance, however, declined by an average rate of about $14 \%$ from both the optimistic and pessimistic perspectives.

As illustrated in Table 8 and Fig. 5 (b), no province technically showed any progress in road safety performance from 2015 to 2016, as $O M P I \leq 1$ and $P M P I \leq 1$. This is mainly because of a significant decrease in average technical change over the period of time from 20152016, with $42.05 \%$ optimistic decline and a $45.81 \%$ pessimistic drop. On average, the efficiency of Iranian provinces optimistically progressed by about $7 \%$ from 


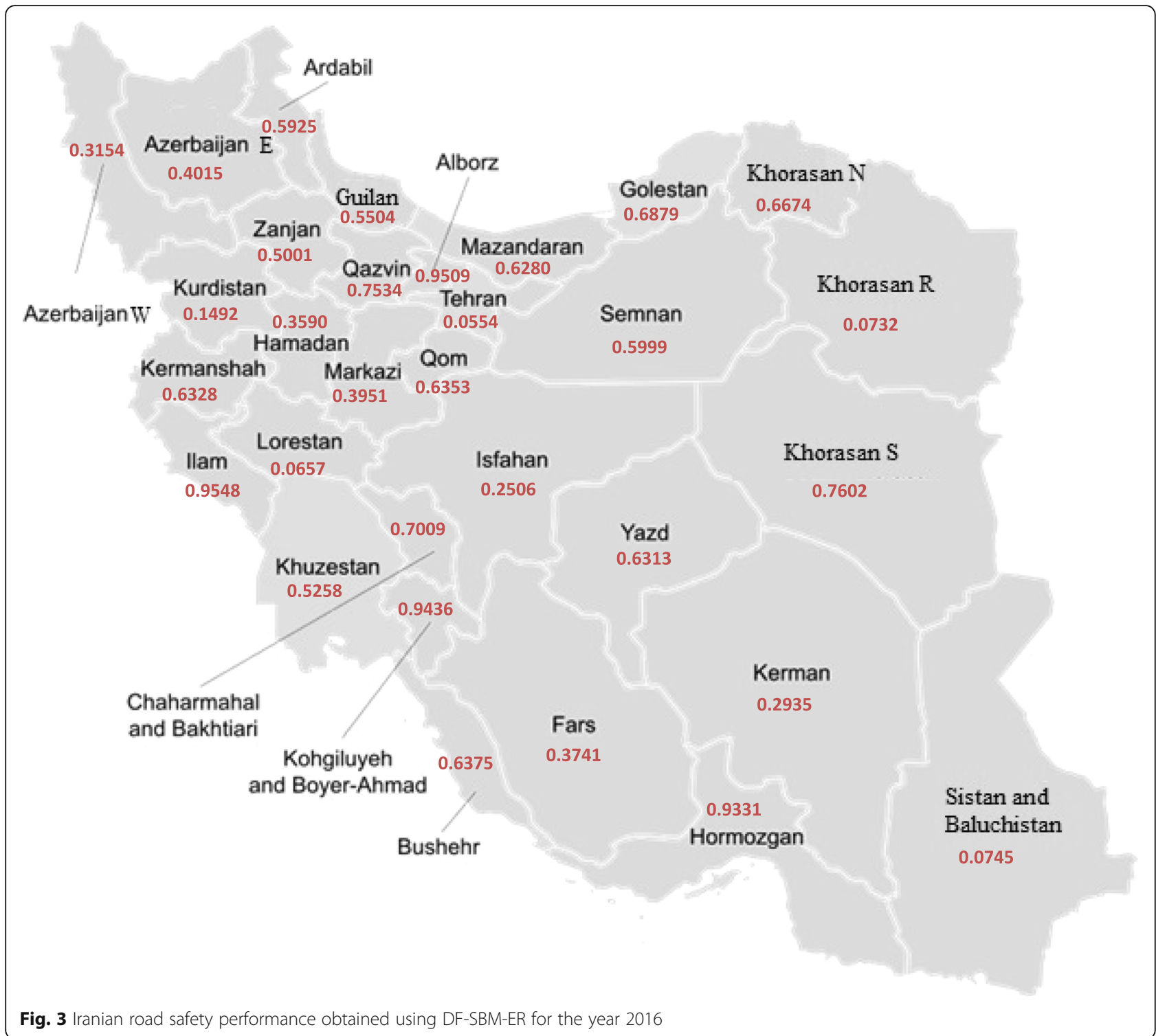

2015 to 2016; meanwhile, Iranian provinces pessimistically showed an increase of nearly $19 \%$.

The rather different results can be addressed by computing the integrated OMPI and PMPI. For this purpose, double frontier MPIs are obtained using a geometric integration of OMPIs and PMPIs through Eq. (27). Table 9 and Fig. 6 provide the achieved double frontier MPIs with associated components, efficiency and technical changes, for all Iranian provinces in terms of road safety performance from 2014-2016. Although technical changes in Iranian provinces optimistically dropped by an average of 2.43\% between 2014 and 2015 (Table 7), the double frontier MPI shows an average rise of about $16 \%$.

Fig. 6 graphically displays the double frontier MPIs for two-year (2014-2015 and 2015-2016) and three-year (2014-2016) evaluations. As illustrated in Table 9 and
Fig. 6, the overall double frontier MPI demonstrates that most Iranian provinces were unproductive in road safety performance from 2014 to 2016 . Iranian road safety performance declined by an average of $26.06 \%$, mainly due to technical negative changes (19.39\% decrease), over the time period 2014-2016. During the time period 2015-2016, the road safety performance of Iranian provinces dropped by an average rate of $36.68 \%$, mainly due to a significant reduction of about $44 \%$ in technical changes, while the average integrated efficiency progressed with a mean rate of around 13\%. During the time period 2014-2015, Iranian road safety performance declined by an average of $13.66 \%$, largely owing to a considerable decrease of $25.54 \%$ in efficiency changes, while the integrated technical changes improved at a mean rate of about $16 \%$. 
Table 6 Comparisons between the Efficiency results for Iranian road safety performance obtained using DF-SBM-ER and DF-CCRER [15] methods

\begin{tabular}{|c|c|c|c|c|c|c|c|c|c|c|c|c|}
\hline \multirow[t]{3}{*}{ Province } & \multicolumn{4}{|l|}{2014} & \multicolumn{4}{|l|}{2015} & \multicolumn{4}{|l|}{2016} \\
\hline & \multicolumn{2}{|c|}{ DF-CCR-ER } & \multicolumn{2}{|l|}{ DF-SBM-ER } & \multicolumn{2}{|l|}{ DF-CCR-ER } & \multicolumn{2}{|l|}{ DF-SBM-ER } & \multicolumn{2}{|l|}{ DF-CCR-ER } & \multicolumn{2}{|l|}{ DF-SBM-ER } \\
\hline & Efficiency & Rank & Efficiency & $\overline{\text { Rank }}$ & Efficiency & Rank & Efficiency & Rank & Efficiency & Rank & Efficiency & Rank \\
\hline Azerbaijan E & 0.3253 & 24 & 0.4079 & 24 & 0.3362 & 20 & 0.3405 & 21 & 0.2952 & 22 & 0.4015 & 20 \\
\hline Azerbaijan W & 0.3463 & 23 & 0.4952 & 21 & 0.2656 & 24 & 0.3515 & 20 & 0.2104 & 25 & 0.3154 & 24 \\
\hline Ardabil & 0.1126 & 29 & 0.0875 & 29 & 0.4106 & 14 & 0.5110 & 15 & 0.6326 & 14 & 0.5925 & 16 \\
\hline Isfahan & 0.3918 & 21 & 0.4789 & 22 & 0.3411 & 19 & 0.2988 & 23 & 0.1364 & 27 & 0.2506 & 26 \\
\hline Alborz & 0.9289 & 2 & 0.9622 & 1 & 0.9197 & 1 & 0.9497 & 1 & 0.9348 & 1 & 0.9509 & 2 \\
\hline Ilam & 0.8053 & 8 & 0.9254 & 7 & 0.7952 & 6 & 0.9024 & 3 & 0.9049 & 2 & 0.9548 & 1 \\
\hline Bushehr & 0.5988 & 12 & 0.6285 & 16 & 0.6627 & 9 & 0.6349 & 8 & 0.6492 & 13 & 0.6375 & 10 \\
\hline Tehran & 0.7614 & 11 & 0.9350 & 6 & 0.3876 & 15 & 0.5619 & 12 & 0.0669 & 31 & 0.0554 & 31 \\
\hline Chaharmahal and Bakhtiari & 0.8134 & 7 & 0.7723 & 11 & 0.8027 & 5 & 0.8938 & 5 & 0.5905 & 16 & 0.7009 & 7 \\
\hline Khorasan S & 0.8974 & 3 & 0.9422 & 4 & 0.6862 & 8 & 0.6061 & 9 & 0.8260 & 6 & 0.7602 & 5 \\
\hline Khorasan R & 0.1823 & 27 & 0.2897 & 28 & 0.093 & 30 & 0.0591 & 30 & 0.0914 & 28 & 0.0732 & 29 \\
\hline Khorasan N & 0.8215 & 5 & 0.7755 & 10 & 0.6196 & 10 & 0.5691 & 11 & 0.6889 & 9 & 0.6674 & 9 \\
\hline Khuzestan & 0.8014 & 9 & 0.9356 & 5 & 0.719 & 7 & 0.8914 & 6 & 0.5897 & 17 & 0.5258 & 18 \\
\hline Zanjan & 0.2718 & 25 & 0.3366 & 27 & 0.3508 & 17 & 0.3633 & 19 & 0.4795 & 19 & 0.5001 & 19 \\
\hline Semnan & 0.5688 & 16 & 0.6199 & 17 & 0.2918 & 23 & 0.3963 & 17 & 0.6559 & 12 & 0.5999 & 15 \\
\hline Sistan and Baluchistan & 0.0613 & 31 & 0.0448 & 31 & 0.1303 & 27 & 0.0767 & 28 & 0.0832 & 29 & 0.0745 & 28 \\
\hline Fars & 0.7827 & 10 & 0.9075 & 8 & 0.3437 & 18 & 0.3404 & 22 & 0.3343 & 21 & 0.3741 & 22 \\
\hline Qazvin & 0.8973 & 4 & 0.9570 & 3 & 0.5304 & 12 & 0.5882 & 10 & 0.8273 & 5 & 0.7534 & 6 \\
\hline Qom & 0.5051 & 18 & 0.6876 & 12 & 0.2972 & 22 & 0.1629 & 25 & 0.6868 & 10 & 0.6353 & 11 \\
\hline Kurdistan & 0.1313 & 28 & 0.3758 & 25 & 0.1591 & 26 & 0.0955 & 27 & 0.1897 & 26 & 0.1492 & 27 \\
\hline Kerman & 0.3717 & 22 & 0.4147 & 23 & 0.1001 & 29 & 0.0681 & 29 & 0.2477 & 23 & 0.2935 & 25 \\
\hline Kermamshah & 0.4929 & 19 & 0.5575 & 20 & 0.5547 & 11 & 0.5388 & 13 & 0.5982 & 15 & 0.6328 & 12 \\
\hline Kohgiluyeh and Boyer-Ahmad & 0.5853 & 14 & 0.6186 & 18 & 0.871 & 3 & 0.9136 & 2 & 0.8818 & 4 & 0.9436 & 3 \\
\hline Golestan & 0.5505 & 17 & 0.6438 & 14 & 0.427 & 13 & 0.5360 & 14 & 0.7139 & 8 & 0.6879 & 8 \\
\hline Guilan & 0.4532 & 20 & 0.5755 & 19 & 0.302 & 21 & 0.3956 & 18 & 0.5543 & 18 & 0.5504 & 17 \\
\hline Lorestan & 0.0675 & 30 & 0.0543 & 30 & 0.0812 & 31 & 0.0590 & 31 & 0.0791 & 30 & 0.0657 & 30 \\
\hline Mazandaran & 0.5878 & 13 & 0.6710 & 13 & 0.3778 & 16 & 0.4916 & 16 & 0.7595 & 7 & 0.6280 & 14 \\
\hline Markazi & 0.5827 & 15 & 0.6410 & 15 & 0.1968 & 25 & 0.1122 & 26 & 0.3491 & 20 & 0.3951 & 21 \\
\hline Hormozgan & 0.9335 & 1 & 0.9584 & 2 & 0.879 & 2 & 0.9017 & 4 & 0.8869 & 3 & 0.9331 & 4 \\
\hline Hamedan & 0.1839 & 26 & 0.3591 & 26 & 0.1265 & 28 & 0.2156 & 24 & 0.2269 & 24 & 0.3590 & 23 \\
\hline Yazd & 0.8162 & 6 & 0.8040 & 9 & 0.8378 & 4 & 0.7265 & 7 & 0.6600 & 11 & 0.6313 & 13 \\
\hline
\end{tabular}

As shown in Fig. 6 and Table 9, only three provinces, Ardabil, Zanjan and Kohgiluyeh-and-Boyer-Ahmad, progressed in road safety performance with growth rates of $32.95 \%, 3.27 \%$ and $10.44 \%$ respectively. The main reason for their improvement was efficiency enhancement, while they experienced a decline in technology from 2014 to 2016. On the other hand, Tehran experienced the most negative growth in road safety performance during the three-year evaluation (2014-2016) by taking into account the optimistic and pessimistic points of view, with a mean rate of $66.96 \%$ decline in MPI; meanwhile, it achieved a negative growth not only in efficiency but also in technology, with mean rates of $60.67 \%$ (decrease) and $16 \%$ (decrease) respectively. Therefore, it can be concluded that Tehran's negative growth in road safety performance was mainly influenced by negative changes in efficiency, since it is technically recognized as one of the top six provinces. As clearly shown in Tables 3,4 and 5, the efficiency of Tehran optimistically declined from 1.00 (super efficiency of 1.0349 ) (rank $1^{\text {st }}$ ) in 2014 to 0.4281 (rank $12^{\text {th }}$ ) in 2015 and then to 0.1575 (rank $31^{\text {th }}$ ) in 2016 . From the pessimistic point of view, the efficiency of Tehran decreased from 
a) 2014

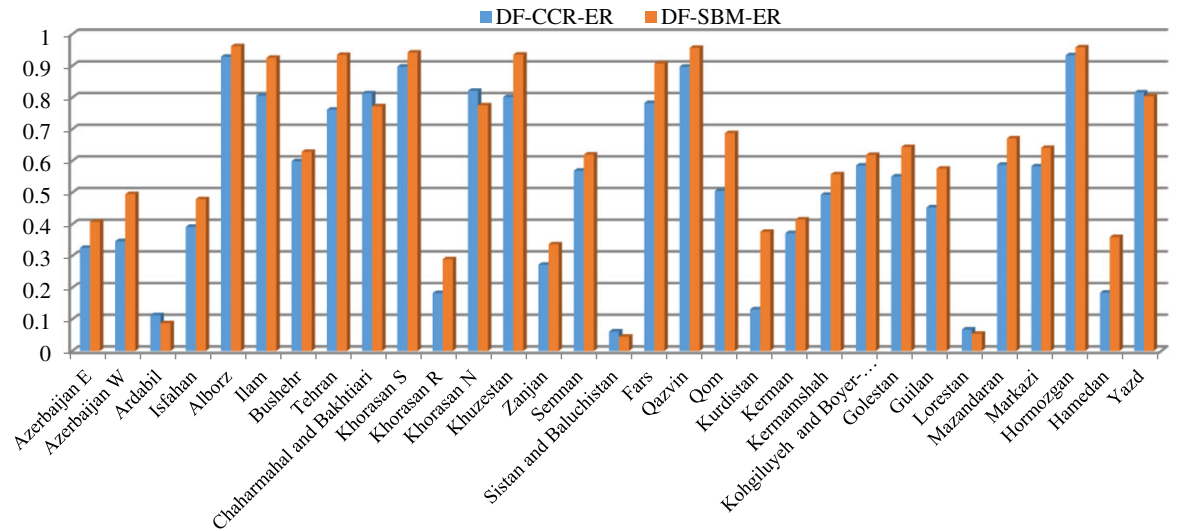

b) 2015

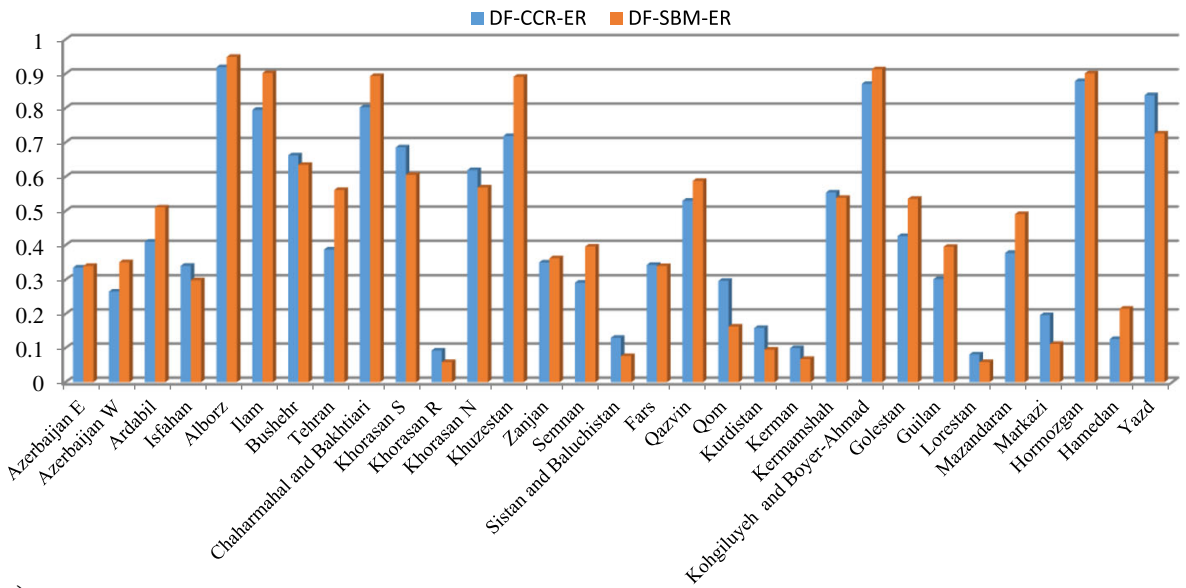

c) 2016

DF-CCR-ER $\quad$ DF-SBM-ER

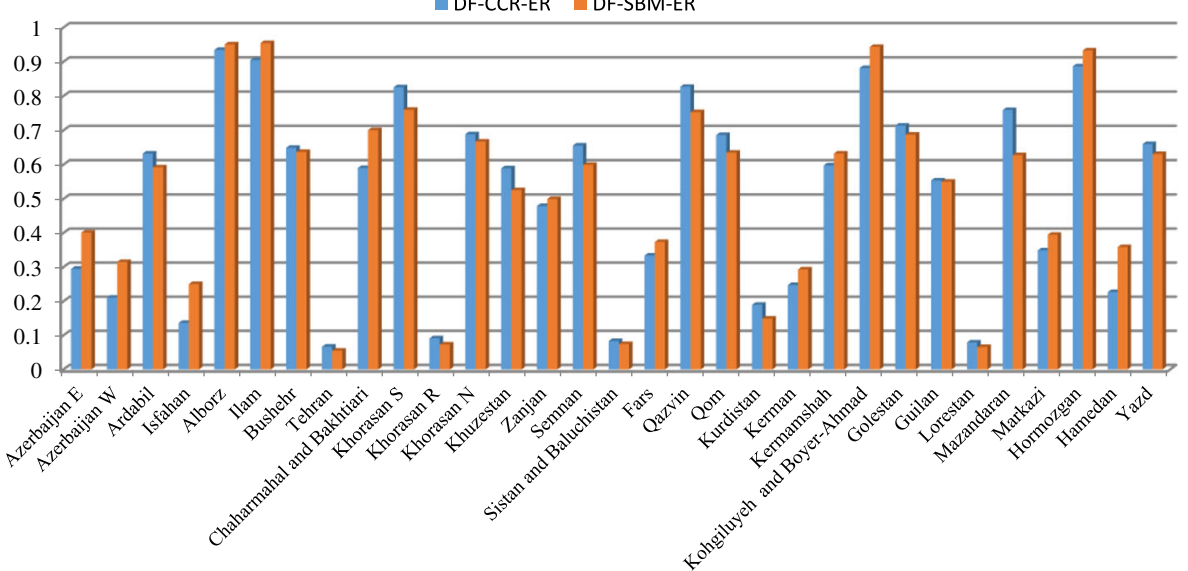

Fig. 4 Comparisons between the efficiency results obtained using DF-CCR-ER and DF-SBM-ER [15] methods

$0.8168\left(\operatorname{rank} 8^{\text {th }}\right)$ in 2014 to $0.6740\left(\right.$ rank $\left.11^{\text {th }}\right)$ in 2015 and then to $0.00\left(\right.$ rank $\left.27^{\text {th }}\right)$ in 2016.

More precisely, the MPI values alone are not enough to evaluate a province in terms of road safety performance, and a province should also be productive with respect to both technical and efficiency components. Figures 7, 8 and 9 depict the status of Iranian provinces in terms of road safety performance in Cartesian coordinates, with efficiency changes as the vertical axis and technical changes as the horizontal axis. The coordinates 
Table 7 OMPI and PMPI values for the 31 Iranian provinces (2014-2015)

\begin{tabular}{|c|c|c|c|c|c|c|c|}
\hline \multirow[t]{2}{*}{ Provinces } & $D_{0}^{14}\left(x_{0}^{14}, y_{0}^{14}\right)$ & $D_{0}^{14}\left(x_{0}^{15}, y_{0}^{15}\right)$ & $D_{0}^{15}\left(x_{0}^{15}, y_{0}^{15}\right)$ & $D_{0}^{15}\left(x_{0}^{14}, y_{0}^{14}\right)$ & OEC & OTC & OMPI \\
\hline & $\tilde{D}_{0}^{14}\left(x_{0}^{14}, y_{0}^{14}\right)$ & $\tilde{\mathrm{D}}_{0}^{14}\left(\mathbf{x}_{0}^{15}, \mathbf{y}_{0}^{15}\right)$ & $\tilde{D}_{0}^{15}\left(x_{0}^{15}, y_{0}^{15}\right)$ & $\tilde{D}_{0}^{15}\left(x_{0}^{14}, y_{0}^{14}\right)$ & & & \\
\hline \multicolumn{8}{|l|}{ OMPI } \\
\hline Azerbaijan E & 0.2828 & 0.2870 & 0.2863 & 0.2830 & 1.0125 & 1.0008 & 1.0133 \\
\hline Azerbaijan W & 0.3427 & 0.3049 & 0.2927 & 0.3297 & 0.8541 & 1.0404 & 0.8886 \\
\hline Ardabil & 0.2414 & 0.4168 & 0.3970 & 0.2300 & 1.6444 & 1.0496 & 1.7260 \\
\hline Isfahan & 0.3308 & 0.2710 & 0.2740 & 0.3373 & 0.8284 & 0.9848 & 0.8158 \\
\hline Alborz & 1.2176 & 1.2753 & 1.7734 & 0.9535 & 1.4565 & 0.9583 & 1.3957 \\
\hline Ilam & 1.0467 & 0.9334 & 1.1475 & 1.1850 & 1.0963 & 0.8476 & 0.9293 \\
\hline Bushehr & 0.4626 & 0.5409 & 0.5171 & 0.4429 & 1.1179 & 1.0453 & 1.1684 \\
\hline Tehran & 1.0349 & 0.4502 & 0.4281 & 1.0417 & 0.4136 & 1.0221 & 0.4228 \\
\hline Chaharmahal and Bakhtiari & 0.6986 & 0.6110 & 1.0309 & 1.0492 & 1.4757 & 0.6282 & 0.9270 \\
\hline Khorasan S & 1.0307 & 0.5081 & 0.5001 & 1.0616 & 0.4852 & 0.9932 & 0.4819 \\
\hline Khorasan R & 0.2126 & 0.1705 & 0.1673 & 0.2132 & 0.7872 & 1.0078 & 0.7934 \\
\hline Khorasan N & 0.6563 & 0.4767 & 0.4571 & 0.6751 & 0.6965 & 1.0069 & 0.7013 \\
\hline Khuzestan & 1.0125 & 0.5557 & 1.0381 & 1.0681 & 1.0253 & 0.7123 & 0.7303 \\
\hline Zanjan & 0.2332 & 0.3123 & 0.3010 & 0.2247 & 1.2909 & 1.0375 & 1.3394 \\
\hline Semnan & 0.4515 & 0.3326 & 0.3173 & 0.4315 & 0.7027 & 1.0474 & 0.7360 \\
\hline Sistan and Baluchistan & 0.1287 & 0.1952 & 0.2136 & 0.1314 & 1.6601 & 0.9459 & 1.5703 \\
\hline Fars & 1.0433 & 0.2946 & 0.2824 & 1.0691 & 0.2707 & 1.0091 & 0.2731 \\
\hline Qazvin & 1.0269 & 0.4847 & 0.4634 & 1.0210 & 0.4513 & 1.0257 & 0.4629 \\
\hline Qom & 0.5263 & 0.4422 & 0.4203 & 0.5006 & 0.7986 & 1.0517 & 0.8399 \\
\hline Kurdistan & 0.2649 & 0.2674 & 0.2615 & 0.2584 & 0.9874 & 1.0238 & 1.0109 \\
\hline Kerman & 0.2856 & 0.2003 & 0.1914 & 0.2728 & 0.6701 & 1.0467 & 0.7014 \\
\hline Kermamshah & 0.3804 & 0.4431 & 0.4265 & 0.3665 & 1.1211 & 1.0384 & 1.1642 \\
\hline Kohgiluyeh and Boyer-Ahmad & 0.4649 & 0.6942 & 1.0360 & 0.4577 & 2.2285 & 0.8249 & 1.8384 \\
\hline Golestan & 0.4712 & 0.4334 & 0.4128 & 0.4491 & 0.8761 & 1.0495 & 0.9195 \\
\hline Guilan & 0.3988 & 0.3314 & 0.3162 & 0.3809 & 0.7931 & 1.0474 & 0.8307 \\
\hline Lorestan & 0.1545 & 0.1744 & 0.1672 & 0.1482 & 1.0822 & 1.0426 & 1.1283 \\
\hline Mazandaran & 0.5023 & 0.4069 & 0.3851 & 0.4760 & 0.7668 & 1.0558 & 0.8096 \\
\hline Markazi & 0.4632 & 0.3168 & 0.3026 & 0.4436 & 0.6534 & 1.0455 & 0.6831 \\
\hline Hormozgan & 1.2585 & 0.6100 & 1.0160 & 1.2320 & 0.8073 & 0.7831 & 0.6322 \\
\hline Hamedan & 0.2456 & 0.2388 & 0.2295 & 0.2361 & 0.9345 & 1.0404 & 0.9723 \\
\hline Yazd & 0.6968 & 0.6599 & 0.6286 & 0.6669 & 0.9021 & 1.0473 & 0.9448 \\
\hline Mean & & & & & 0.8839 & 0.9757 & 0.8625 \\
\hline \multirow[t]{2}{*}{ Provinces } & $d_{0}^{14}\left(x_{0}^{14}, y_{0}^{14}\right)$ & $d_{0}^{14}\left(x_{0}^{15}, y_{0}^{15}\right)$ & $d_{0}^{15}\left(x_{0}^{15}, y_{0}^{15}\right)$ & $d_{0}^{15}\left(x_{0}^{14}, y_{0}^{14}\right)$ & PEC & PTC & PMPI \\
\hline & $d_{0}^{14}\left(x_{0}^{14}, y_{0}^{14}\right)$ & $\tilde{d}_{0}^{14}\left(x_{0}^{15}, y_{0}^{15}\right)$ & $\tilde{d}_{0}^{15}\left(x_{0}^{15}, y_{0}^{15}\right)$ & $\tilde{d}_{0}^{15}\left(x_{0}^{14}, y_{0}^{14}\right)$ & & & \\
\hline \multicolumn{8}{|l|}{ PMPI } \\
\hline Azerbaijan E & 2.3009 & 2.3417 & 1.8027 & 1.7792 & 0.7835 & 1.2961 & 1.0155 \\
\hline Azerbaijan W & 2.8521 & 2.5745 & 1.8450 & 2.0620 & 0.6469 & 1.3893 & 0.8987 \\
\hline Ardabil & 0.9797 & 3.9403 & 2.6402 & 0.9721 & 2.6951 & 1.2264 & 3.3052 \\
\hline Isfahan & 2.7331 & 2.2582 & 1.6224 & 2.0312 & 0.5936 & 1.3685 & 0.8124 \\
\hline Alborz & 9.1483 & 9.6453 & 6.9547 & 6.5986 & 0.7602 & 1.3866 & 1.0542 \\
\hline Ilam & 4.8018 & 4.3584 & 3.7500 & 4.0278 & 0.7810 & 1.1771 & 0.9193 \\
\hline
\end{tabular}


Table 7 OMPI and PMPI values for the 31 Iranian provinces (2014-2015) (Continued)

\begin{tabular}{|c|c|c|c|c|c|c|c|}
\hline Bushehr & 4.0002 & 2.7508 & 3.4279 & 2.9205 & 0.8569 & 1.0484 & 0.8984 \\
\hline Tehran & 5.4571 & 4.2718 & 3.0670 & 4.2371 & 0.5620 & 1.3393 & 0.7527 \\
\hline Chaharmahal and Bakhtiari & 4.4454 & 4.3437 & 3.4720 & 3.5808 & 0.7810 & 1.2462 & 0.9734 \\
\hline Khorasan S & 6.0988 & 4.1753 & 3.0937 & 4.6462 & 0.5073 & 1.3310 & 0.6752 \\
\hline Khorasan R & 1.7587 & 0.9927 & 0.9089 & 1.2701 & 0.5168 & 1.2297 & 0.6355 \\
\hline Khorasan N & 5.5964 & 4.0740 & 2.9191 & 3.9972 & 0.5216 & 1.3979 & 0.7291 \\
\hline Khuzestan & 5.5058 & 4.8922 & 3.4037 & 3.8011 & 0.6182 & 1.4429 & 0.8920 \\
\hline Zanjan & 1.9768 & 2.6323 & 1.8868 & 1.3178 & 0.9544 & 1.4466 & 1.3807 \\
\hline Semnan & 3.9429 & 3.0174 & 2.0413 & 2.8193 & 0.5177 & 1.4378 & 0.7444 \\
\hline Sistan and Baluchistan & 0.7627 & 0.9300 & 0.9427 & 0.7609 & 1.2361 & 0.9944 & 1.2291 \\
\hline Fars & 3.9377 & 2.4512 & 1.8157 & 3.3864 & 0.4611 & 1.2529 & 0.5777 \\
\hline Qazvin & 8.0790 & 2.4512 & 3.1530 & 5.6208 & 0.3903 & 1.0571 & 0.4125 \\
\hline Qom & 4.7212 & 3.7199 & 0.9994 & 3.3855 & 0.2117 & 2.2783 & 0.4823 \\
\hline Kurdistan & 2.1244 & 2.1420 & 0.9609 & 0.9580 & 0.4523 & 2.2233 & 1.0057 \\
\hline Kerman & 2.3477 & 1.6414 & 0.9895 & 1.7472 & 0.4215 & 1.4929 & 0.6292 \\
\hline Kermamshah & 3.4888 & 3.9413 & 2.7598 & 2.3755 & 0.7910 & 1.4482 & 1.1456 \\
\hline Kohgiluyeh and Boyer-Ahmad & 3.7229 & 5.5251 & 4.1917 & 2.7982 & 1.1259 & 1.3243 & 1.4910 \\
\hline Golestan & 4.2919 & 3.9702 & 2.8295 & 3.0585 & 0.6592 & 1.4032 & 0.9251 \\
\hline Guilan & 3.6343 & 2.8922 & 2.0408 & 2.4890 & 0.5615 & 1.4385 & 0.8078 \\
\hline Lorestan & 0.9500 & 1.2498 & 0.9032 & 0.8539 & 0.9508 & 1.2407 & 1.1796 \\
\hline Mazandaran & 4.6067 & 3.2851 & 2.5066 & 3.0967 & 0.5441 & 1.3963 & 0.7598 \\
\hline Markazi & 4.3466 & 2.3358 & 0.9889 & 3.0433 & 0.2275 & 1.8367 & 0.4179 \\
\hline Hormozgan & 8.3428 & 5.0643 & 3.7247 & 6.0590 & 0.4465 & 1.3683 & 0.6109 \\
\hline Hamedan & 2.0858 & 2.0317 & 1.3760 & 1.4272 & 0.6597 & 1.4690 & 0.9691 \\
\hline Yazd & 6.1221 & 5.7688 & 4.1485 & 4.2521 & 0.6776 & 1.4149 & 0.9588 \\
\hline Mean & & & & & 0.6273 & 1.3779 & 0.8643 \\
\hline
\end{tabular}

of the origin is also defined as the point $(1,1)$, where the vertical and technical axes intersect.

Although the optimistic MPIs indicate that ten provinces are productive in road safety performance (Table 7 ), Fig. 7. a displays that six provinces were located in the $1^{\text {st }}$ quadrant, which means that these provinces optimistically increased their efficiency and technology from 20142015. Pessimistically, only two provinces, Ardabil and Kohgiluyeh-and-Boyer-Ahmad, were located in the $1^{\text {st }}$ quadrant (Fig. 7.b), and the pessimistic MPI (Table 7) shows that nine provinces are productive. As shown in Fig. 7.c, the double-frontier assessment also demonstrates that five provinces simultaneously enhanced their productivity on road safety, taking into account both components, from 2014-2015. Briefly, the Iranian provinces were mostly productive in terms of technological advancement, since most provinces were in the $1^{\text {st }}$ and $2^{\text {nd }}$ quadrants from 2014-2015.

As shown in Fig. 8 (a) -(c), unfortunately, no provinces were positioned in the first quadrant, which means that all provinces declined their productivity from 2015-
2016. On the other hand, most provinces were in the third quadrant, which means that Iranian provinces were mostly productive in terms of efficiency enhancement in 2015-2016.

Generally speaking, the three-year assessment from 2014 to 2016, as illustrated in Fig. 9, indicates that most Iranian provinces were located in the $3^{\text {th }}$ and $4^{\text {th }}$ quadrants meaning that they are in general unsuccessful in road safety performance in terms of technology advancement; meanwhile, eleven provinces progressed in road safety performance in terms of efficiency improvement.

For more discussion, the input and output slack variables can be further analyzed. For example, the existing data set, slack values and the deviations of input and output variables for Qom province based on the efficient frontier are shown in Tables 10, 11 and 12 respectively. As illustrated, the maximum deviation for Qom province is related to the input variable $R L S$ with a deviation of about $67 \%$. The results also demonstrate that there is no deviation in the input variable of EMS. Similarly, the relative deviation of input and output variables for all 
Table 8 OMPI and PMPI values for the 31 Iranian provinces (2015-2016)

\begin{tabular}{|c|c|c|c|c|c|c|c|}
\hline Provinces & $\begin{array}{l}D_{0}^{15}\left(x_{0}^{15}, y_{0}^{15}\right) \\
\tilde{D}_{0}^{15}\left(\boldsymbol{x}_{0}^{15}, \boldsymbol{y}_{0}^{15}\right)\end{array}$ & $\begin{array}{l}D_{0}^{15}\left(x_{0}^{16}, y_{0}^{16}\right) \\
\tilde{D}_{0}^{15}\left(\boldsymbol{x}_{0}^{16}, \boldsymbol{y}_{0}^{16}\right)\end{array}$ & $\begin{array}{l}D_{0}^{16}\left(x_{0}^{16}, y_{0}^{16}\right) \\
\tilde{D}_{0}^{16}\left(\boldsymbol{x}_{0}^{16}, \boldsymbol{y}_{0}^{16}\right)\end{array}$ & $\begin{array}{l}D_{0}^{16}\left(x_{0}^{15}, y_{0}^{15}\right) \\
\tilde{D}_{0}^{16}\left(\boldsymbol{x}_{0}^{15}, \boldsymbol{y}_{0}^{15}\right)\end{array}$ & OEC & OTC & OMPI \\
\hline \multicolumn{8}{|l|}{ OMPI } \\
\hline Azerbaijan E & 0.2863 & 0.1908 & 0.3343 & 0.5138 & 1.1676 & 0.5640 & 0.6585 \\
\hline Azerbaijan W & 0.2927 & 0.1617 & 0.2812 & 0.5291 & 0.9606 & 0.5641 & 0.5419 \\
\hline Ardabil & 0.3970 & 0.2940 & 0.4724 & 0.6423 & 1.1899 & 0.6202 & 0.7380 \\
\hline Isfahan & 0.2740 & 0.1480 & 0.2353 & 0.4553 & 0.8587 & 0.6152 & 0.5282 \\
\hline Alborz & 1.7734 & 0.6387 & 1.5789 & 2.1783 & 0.8903 & 0.5739 & 0.5109 \\
\hline Ilam & 1.1475 & 1.0624 & 1.3190 & 1.3890 & 1.1494 & 0.8158 & 0.9376 \\
\hline Bushehr & 0.5171 & 0.3292 & 0.5236 & 1.0139 & 1.0125 & 0.5663 & 0.5733 \\
\hline Tehran & 0.4281 & 0.0988 & 0.1575 & 1.0094 & 0.3679 & 0.5157 & 0.1897 \\
\hline Chaharmahal and Bakhtiari & 1.0309 & 0.3343 & 0.6007 & 1.0945 & 0.5827 & 0.7240 & 0.4219 \\
\hline Khorasan S & 0.5001 & 0.4181 & 0.6784 & 1.0646 & 1.3565 & 0.5381 & 0.7299 \\
\hline Khorasan R & 0.1673 & 0.1249 & 0.2047 & 0.2801 & 1.2233 & 0.6039 & 0.7387 \\
\hline Khorasan N & 0.4571 & 0.3370 & 0.5569 & 0.7962 & 1.2183 & 0.5894 & 0.7181 \\
\hline Khuzestan & 1.0381 & 0.2455 & 0.4288 & 1.2626 & 0.4131 & 0.6862 & 0.2834 \\
\hline Zanjan & 0.3010 & 0.2409 & 0.3922 & 0.5116 & 1.3030 & 0.6011 & 0.7832 \\
\hline Semnan & 0.3173 & 0.3053 & 0.4869 & 0.5058 & 1.5347 & 0.6271 & 0.9625 \\
\hline Sistan and Baluchistan & 0.2136 & 0.1267 & 0.2079 & 0.3412 & 0.9732 & 0.6176 & 0.6011 \\
\hline Fars & 0.2824 & 0.1850 & 0.2984 & 0.4959 & 1.0566 & 0.5941 & 0.6278 \\
\hline Qazvin & 0.4634 & 0.3990 & 0.6651 & 1.0423 & 1.4351 & 0.5165 & 0.7412 \\
\hline Qom & 0.4203 & 0.3252 & 0.5034 & 1.0056 & 1.1977 & 0.5196 & 0.6223 \\
\hline Kurdistan & 0.2615 & 0.2211 & 0.3895 & 0.4603 & 1.4892 & 0.5679 & 0.8457 \\
\hline Kerman & 0.1914 & 0.1552 & 0.2612 & 0.3279 & 1.3649 & 0.5890 & 0.8038 \\
\hline Kermamshah & 0.4265 & 0.2913 & 0.5306 & 1.0175 & 1.2441 & 0.4797 & 0.5968 \\
\hline Kohgiluyeh and Boyer-Ahmad & 1.0360 & 0.5375 & 1.1429 & 1.2945 & 1.1032 & 0.6135 & 0.6768 \\
\hline Golestan & 0.4128 & 0.3147 & 0.6022 & 1.0148 & 1.4589 & 0.4610 & 0.6726 \\
\hline Guilan & 0.3162 & 0.2589 & 0.4472 & 0.5545 & 1.4141 & 0.5746 & 0.8125 \\
\hline Lorestan & 0.1672 & 0.1122 & 0.1849 & 0.3008 & 1.1056 & 0.5809 & 0.6422 \\
\hline Mazandaran & 0.3851 & 0.3095 & 0.5226 & 1.0263 & 1.3570 & 0.4714 & 0.6397 \\
\hline Markazi & 0.3026 & 0.1894 & 0.3209 & 0.5100 & 1.0604 & 0.5919 & 0.6276 \\
\hline Hormozgan & 1.0160 & 0.5313 & 1.1155 & 1.3389 & 1.0979 & 0.6012 & 0.6601 \\
\hline Hamedan & 0.2295 & 0.1696 & 0.3021 & 0.4176 & 1.3165 & 0.5554 & 0.7311 \\
\hline Yazd & 0.6286 & 0.3213 & 0.5235 & 1.2915 & 0.8328 & 0.5465 & 0.4551 \\
\hline Mean & & & & & 1.0706 & 0.5795 & 0.6204 \\
\hline \multirow[t]{2}{*}{ Provinces } & $d_{0}^{15}\left(x_{0}^{15}, y_{0}^{15}\right)$ & $d_{0}^{15}\left(x_{0}^{16}, y_{0}^{16}\right)$ & $d_{0}^{16}\left(x_{0}^{16}, y_{0}^{16}\right)$ & $d_{0}^{16}\left(x_{0}^{15}, y_{0}^{15}\right)$ & PEC & PTC & PMPI \\
\hline & $d_{0}^{15}\left(x_{0}^{15}, y_{0}^{15}\right)$ & $\tilde{d}_{0}^{15}\left(x_{0}^{16}, y_{0}^{16}\right)$ & $\tilde{d}_{0}^{16}\left(x_{0}^{16}, y_{0}^{16}\right)$ & $\tilde{d}_{0}^{16}\left(x_{0}^{15}, y_{0}^{15}\right)$ & & & \\
\hline \multicolumn{8}{|l|}{ PMPI } \\
\hline Azerbaijan E & 1.8027 & 0.9952 & 2.0065 & 3.4933 & 1.1130 & 0.5059 & 0.5631 \\
\hline Azerbaijan W & 1.8450 & 0.9132 & 1.6839 & 3.3195 & 0.9126 & 0.5490 & 0.5011 \\
\hline Ardabil & 2.6402 & 1.9507 & 3.1363 & 4.2084 & 1.1879 & 0.6247 & 0.7420 \\
\hline Isfahan & 1.6224 & 0.8902 & 1.5026 & 2.9699 & 0.9261 & 0.5689 & 0.5269 \\
\hline Alborz & 6.9547 & 4.5652 & 7.1264 & 10.8206 & 1.0247 & 0.6417 & 0.6575 \\
\hline Ilam & 3.7500 & 3.6444 & 7.7097 & 8.4609 & 2.0559 & 0.4577 & 0.9410 \\
\hline
\end{tabular}


Table 8 OMPI and PMPI values for the 31 Iranian provinces (2015-2016) (Continued)

\begin{tabular}{|c|c|c|c|c|c|c|c|}
\hline Bushehr & 3.4279 & 2.1886 & 3.4073 & 5.6397 & 0.9940 & 0.6248 & 0.6211 \\
\hline Tehran & 3.0670 & 0.6598 & 0.8579 & 4.7441 & 0.2797 & 0.7051 & 0.1972 \\
\hline Chaharmahal and Bakhtiari & 3.4720 & 2.0628 & 3.8560 & 6.8044 & 1.1106 & 0.5225 & 0.5802 \\
\hline Khorasan S & 3.0937 & 2.5899 & 4.3876 & 5.8182 & 1.4182 & 0.5602 & 0.7945 \\
\hline Khorasan R & 0.9089 & 0.7301 & 0.9875 & 1.7403 & 1.0864 & 0.6214 & 0.6751 \\
\hline Khorasan N & 2.9191 & 2.1175 & 3.6344 & 4.9800 & 1.2451 & 0.5844 & 0.7276 \\
\hline Khuzestan & 3.4037 & 0.9210 & 2.5899 & 6.0655 & 0.7609 & 0.4467 & 0.3399 \\
\hline Zanjan & 1.8868 & 1.5159 & 2.5513 & 3.3254 & 1.3522 & 0.5806 & 0.7851 \\
\hline Semnan & 2.0413 & 1.9541 & 3.1181 & 3.2418 & 1.5275 & 0.6282 & 0.9595 \\
\hline Sistan and Baluchistan & 0.9427 & 0.7047 & 0.8512 & 2.1527 & 0.9029 & 0.6021 & 0.5436 \\
\hline Fars & 1.8157 & 0.9518 & 1.9661 & 3.2007 & 1.0828 & 0.5240 & 0.5674 \\
\hline Qazvin & 3.1530 & 2.7874 & 4.3957 & 5.1310 & 1.3941 & 0.6242 & 0.8703 \\
\hline Qom & 0.9994 & 0.9206 & 3.5998 & 4.7038 & 3.6019 & 0.2331 & 0.8396 \\
\hline Kurdistan & 0.9609 & 0.9374 & 0.9770 & 2.3753 & 1.0168 & 0.6230 & 0.6334 \\
\hline Kerman & 0.9895 & 0.9189 & 1.6322 & 2.1255 & 1.6495 & 0.5120 & 0.8445 \\
\hline Kermamshah & 2.7598 & 1.8778 & 3.2442 & 4.7679 & 1.1755 & 0.5788 & 0.6804 \\
\hline Kohgiluyeh and Boyer-Ahmad & 4.1917 & 3.4689 & 6.2438 & 8.0350 & 1.4896 & 0.5384 & 0.8019 \\
\hline Golestan & 2.8295 & 2.0083 & 3.5271 & 4.6297 & 1.2466 & 0.5899 & 0.7354 \\
\hline Guilan & 2.0408 & 1.5210 & 2.7493 & 3.3825 & 1.3472 & 0.5777 & 0.7783 \\
\hline Lorestan & 0.9032 & 0.7246 & 0.9922 & 1.9070 & 1.0985 & 0.5881 & 0.6461 \\
\hline Mazandaran & 2.5066 & 0.9784 & 3.2417 & 4.0088 & 1.2933 & 0.4344 & 0.5618 \\
\hline Markazi & 0.9889 & 0.9340 & 2.0174 & 3.3470 & 2.0401 & 0.3698 & 0.7545 \\
\hline Hormozgan & 3.7247 & 3.0950 & 5.3188 & 7.2127 & 1.4280 & 0.5482 & 0.7828 \\
\hline Hamedan & 1.3760 & 0.9527 & 1.8566 & 2.5482 & 1.3493 & 0.5264 & 0.7103 \\
\hline Yazd & 4.1485 & 2.0537 & 3.2917 & 7.1689 & 0.7935 & 0.6009 & 0.4768 \\
\hline Mean & & & & & 1.1926 & 0.5419 & 0.6462 \\
\hline
\end{tabular}

provinces can be achieved for 2014-2016. The average deviation of input and output variables are demonstrated in Fig. 10. It has to be noted that an efficient province, in this regard, is a province with fewer investments in road safety and, consequently, fewer fatalities. As shown in Fig. 10, Iranian provinces experienced a high deviation in road fatalities; i.e. $75 \%$ on average. The results also demonstrate that the deviation of road fatality rate has been reduced to some extent from 2014 (around 91\%) to 2016 (about 75\%). Regarding the input variables, the highest deviation was related to $E \mathcal{E} V$, which indicates an improper distribution of this variable in provinces, with a deviation of around $58 \%$ in $2016,50 \%$ in 2015 , and $33 \%$ in 2014 . The second highest deviation regarding the input variables in 2015 and 2016 was related to the variable $P S$, with a deviation of more than $30 \%$, compared to a deviation of about $21 \%$ in 2014, which ranked $3^{\text {rd }}$. Furthermore, the lowest deviation in 2016 was related to variable $C$, with a deviation of about $11 \%$, compared to a deviation of more than 16\% in 2014 and $24 \%$ in 2015. This result shows the improved distribution of cameras in 2016. Apart from fatality risk and cameras, the deviation of other variables from ideal situation has increased in 2016 compared to the years 2014 and 2015, which requires special attention from the Iranian government in their future planning. Generally speaking, although the average deviation of the fatality rate has been improved by $17.6 \%$, Iranian provinces have not succeeded in reducing the deviation of input variables over the period 2014-2016.

For more discussion, the deviations of input and output variables for the year 2016 are shown in detail in Fig. 11 . As can be noted, there are no deviations for efficient provinces taking into account all input and output variables (Ilam, Alborz, Kohgiluyeh-and-Boyer-Ahmad, and Hormozgan). In addition to the efficient provinces, Fig. 11.a illustrates that Bushehr and Sistan-and-Baluchistan have also experienced a deviation of less than $10 \%$ in terms 
a) Two-year evaluation (2014-2015)
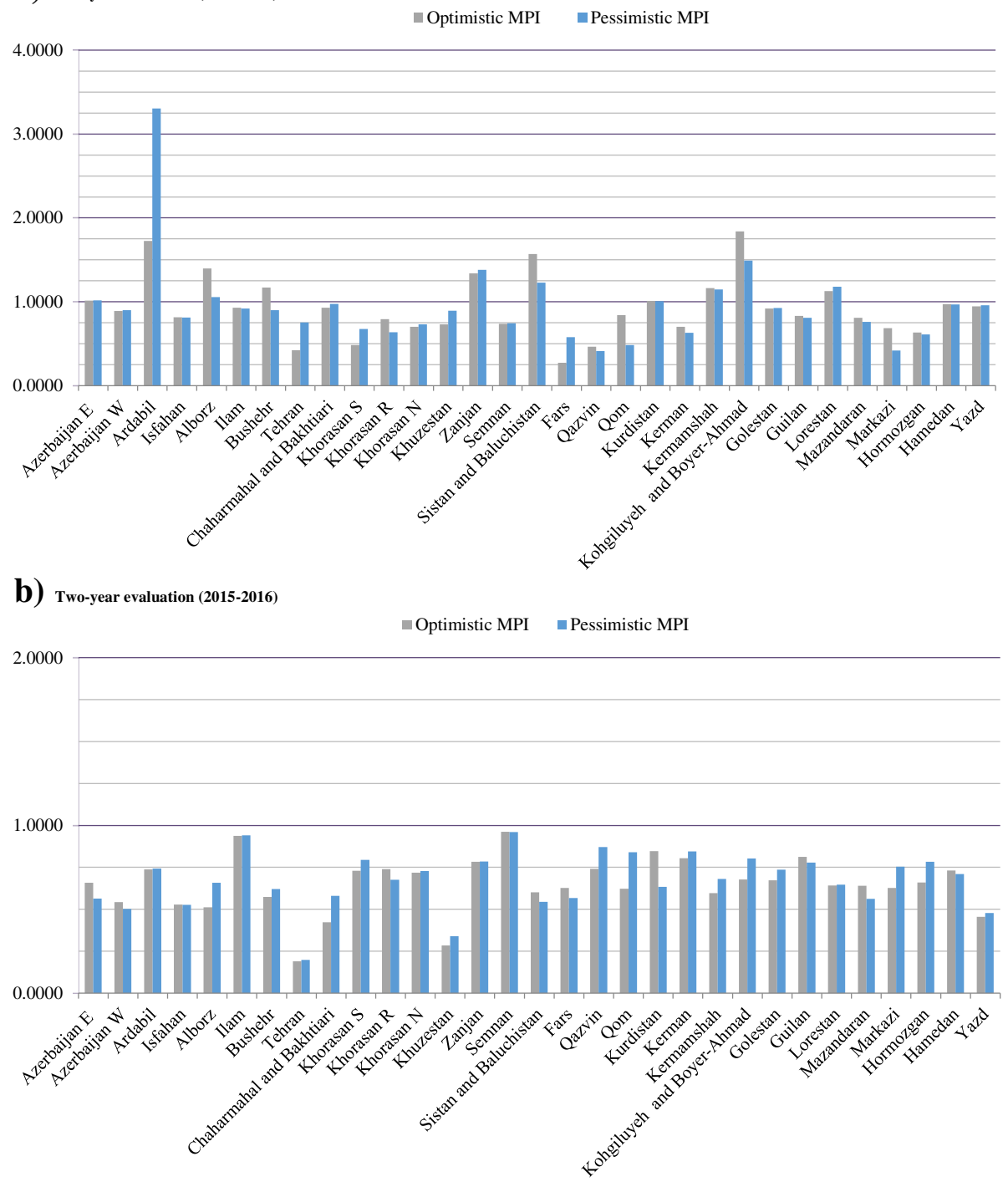

Fig. 5 Comparison of Malmquist productivity index values from the two points of view, (a) optimistic MPI and (b) pessimistic MPI

of PS. As shown in Fig. 11.a, about 25\% of provinces experienced a deviation of more than $50 \%$. It is worth noting that the highest deviation regarding PS variable is related to Khuzestan followed by Kermanshah and Azerbaijan E.

Figure 11.b shows that there is no deviation in $R M D$ for Ardebil, Tehran, Khuzestan, Semnan, and Mazandaran. Apparently, Khuzestan and Mazandaran have experienced no deviation in $R M D$ in contrast to their high deviation in PS. Fig. 11.c also presents that about $77 \%$ of provinces have experienced a deviation of more than $50 \%$ in $E \mathcal{E} V$. As illustrated in Fig. 11.d, about half the provinces have effectively invested in $C$ without any deviation and five provinces have marginally deviated from the ideal position with a deviation of less than $10 \%$.
Although Qom province has received a deviation of more than $50 \%$ in $C$, it has experienced no deviation in EMS (Fig. 11.e). Tehran, Markazi, and Yazd have also invested in EMS effectively. The deviations of about 22\% Iranian provinces from the ideal were more than $50 \%$. Figure 11.e also shows that, similar to $P S$ and $E \mathcal{E} V$, the deviation of Khuzestan province in EMS is critically high and needs to be reconsidered by the authorities. According to Fig. 11.f, any deviation from the ideal is observed for twelve provinces while there is a deviation of more than $50 \%$ for about $25 \%$ of provinces. Figure 11 .g provides some information about road fatality risk. The results confirm that nine provinces have succeeded in reducing road fatalities with no deviation from Ideal; 
Table 9 The double-frontier MPI for the three-year evaluation (2014-2016)

\begin{tabular}{|c|c|c|c|c|c|c|c|c|c|}
\hline \multirow[t]{2}{*}{ Provinces } & \multicolumn{3}{|c|}{$2014 / 2015$} & \multicolumn{3}{|c|}{$2015 / 2016$} & \multicolumn{3}{|c|}{$2014 / 2016$} \\
\hline & $\overline{E C}$ & $T C$ & $M P I$ & $\overline{E C}$ & $T C$ & $M P I$ & $\overline{E C}$ & $T C$ & MPI \\
\hline Azerbaijan E & 0.8907 & 1.1389 & 1.0144 & 1.1400 & 0.5342 & 0.6090 & 1.0076 & 0.7800 & 0.7860 \\
\hline Azerbaijan W & 0.7433 & 1.2023 & 0.8937 & 0.9363 & 0.5565 & 0.5211 & 0.8343 & 0.8180 & 0.6824 \\
\hline Ardabil & 2.1052 & 1.1346 & 2.3885 & 1.1889 & 0.6224 & 0.7400 & 1.5820 & 0.8404 & 1.3295 \\
\hline Isfahan & 0.7012 & 1.1609 & 0.8141 & 0.8918 & 0.5916 & 0.5276 & 0.7908 & 0.8287 & 0.6553 \\
\hline Alborz & 1.0523 & 1.1527 & 1.2130 & 0.9551 & 0.6068 & 0.5796 & 1.0025 & 0.8364 & 0.8385 \\
\hline Ilam & 0.9253 & 0.9989 & 0.9242 & 1.5372 & 0.6111 & 0.9393 & 1.1926 & 0.7813 & 0.9318 \\
\hline Bushehr & 0.9787 & 1.0468 & 1.0246 & 1.0032 & 0.5948 & 0.5967 & 0.9909 & 0.7891 & 0.7819 \\
\hline Tehran & 0.4822 & 1.1700 & 0.5641 & 0.3208 & 0.6030 & 0.1935 & 0.3933 & 0.8400 & 0.3304 \\
\hline Chaharmahal and Bakhtiari & 1.0736 & 0.8848 & 0.9499 & 0.8044 & 0.6150 & 0.4948 & 0.9293 & 0.7377 & 0.6855 \\
\hline Khorasan S & 0.4961 & 1.1497 & 0.5704 & 1.3870 & 0.5490 & 0.7615 & 0.8295 & 0.7945 & 0.6591 \\
\hline Khorasan R & 0.6379 & 1.1132 & 0.7101 & 1.1528 & 0.6126 & 0.7062 & 0.8575 & 0.8258 & 0.7081 \\
\hline Khorasan N & 0.6027 & 1.1864 & 0.7151 & 1.2316 & 0.5869 & 0.7228 & 0.8616 & 0.8344 & 0.7189 \\
\hline Khuzestan & 0.7962 & 1.0138 & 0.8071 & 0.5606 & 0.5536 & 0.3104 & 0.6681 & 0.7492 & 0.5005 \\
\hline Zanjan & 1.1100 & 1.2251 & 1.3599 & 1.3274 & 0.5908 & 0.7842 & 1.2138 & 0.8508 & 1.0327 \\
\hline Semnan & 0.6032 & 1.2272 & 0.7402 & 1.5311 & 0.6277 & 0.9610 & 0.9610 & 0.8776 & 0.8434 \\
\hline Sistan and Baluchistan & 1.4325 & 0.9699 & 1.3893 & 0.9374 & 0.6098 & 0.5716 & 1.1588 & 0.7690 & 0.8912 \\
\hline Fars & 0.3533 & 1.1244 & 0.3972 & 1.0696 & 0.5580 & 0.5968 & 0.6147 & 0.7921 & 0.4869 \\
\hline Qazvin & 0.4197 & 1.0413 & 0.4370 & 1.4145 & 0.5678 & 0.8032 & 0.7705 & 0.7689 & 0.5924 \\
\hline Qom & 0.4112 & 1.5479 & 0.6365 & 2.0770 & 0.3480 & 0.7229 & 0.9241 & 0.7340 & 0.6783 \\
\hline Kurdistan & 0.6683 & 1.5087 & 1.0083 & 1.2305 & 0.5948 & 0.7319 & 0.9068 & 0.9473 & 0.8591 \\
\hline Kerman & 0.5315 & 1.2501 & 0.6644 & 1.5005 & 0.5491 & 0.8239 & 0.8930 & 0.8285 & 0.7398 \\
\hline Kermamshah & 0.9417 & 1.2263 & 1.1549 & 1.2093 & 0.5269 & 0.6372 & 1.0672 & 0.8039 & 0.8579 \\
\hline Kohgiluyeh and-Boyer-Ahmad & 1.5840 & 1.0452 & 1.6556 & 1.2819 & 0.5747 & 0.7367 & 1.4250 & 0.7750 & 1.1044 \\
\hline Golestan & 0.7600 & 1.2136 & 0.9223 & 1.3486 & 0.5215 & 0.7033 & 1.0124 & 0.7955 & 0.8054 \\
\hline Guilan & 0.6673 & 1.2275 & 0.8192 & 1.3802 & 0.5762 & 0.7952 & 0.9597 & 0.8410 & 0.8071 \\
\hline Lorestan & 1.0143 & 1.1374 & 1.1537 & 1.1021 & 0.5845 & 0.6441 & 1.0573 & 0.8153 & 0.8620 \\
\hline Mazandaran & 0.6459 & 1.2142 & 0.7843 & 1.3247 & 0.4525 & 0.5995 & 0.9250 & 0.7413 & 0.6857 \\
\hline Markazi & 0.3855 & 1.3858 & 0.5343 & 1.4708 & 0.4679 & 0.6882 & 0.7530 & 0.8052 & 0.6064 \\
\hline Hormozgan & 0.6003 & 1.0352 & 0.6214 & 1.2521 & 0.5741 & 0.7188 & 0.8670 & 0.7709 & 0.6684 \\
\hline Hamedan & 0.7852 & 1.2362 & 0.9707 & 1.3328 & 0.5407 & 0.7206 & 1.0230 & 0.8176 & 0.8363 \\
\hline Yazd & 0.7819 & 1.2173 & 0.9518 & 0.8129 & 0.5730 & 0.4658 & 0.7972 & 0.8352 & 0.6659 \\
\hline Mean & 0.7446 & 1.1595 & 0.8634 & 1.1299 & 0.5604 & 0.6332 & 0.9173 & 0.8061 & 0.7394 \\
\hline
\end{tabular}

however, fifteen provinces have significantly deviated from ideal with a deviation of more than $50 \%$. So, it can be stated that although Iranian provinces have generally succeeded in fatality reduction in 2016 compared to 2014 and 2015 (Fig. 10), output shortfalls are still considerable.

In summary, the input excesses, as well as the output shortfalls regarding each province, can be highlighted using Figs. 10 and 11, respectively. The findings would also be appropriate for the provincial authorities to address safety issues in their future planning.

\section{Conclusions}

The DEA models have been recently employed as an effective tool to measure road safety performance worldwide. The existing studies mostly applied the CCR and BCC models for safety measurement, which are on the basis of efficiency ratio and neither considered input excess nor output shortfall. In this respect, the SBM is employed in the current study, which not only measures the efficiency ratio but also takes account of slacks. It is also noted that the safety performance of each DMU (countries, states or provinces) has been previously assessed using the traditional CCR model based only on 


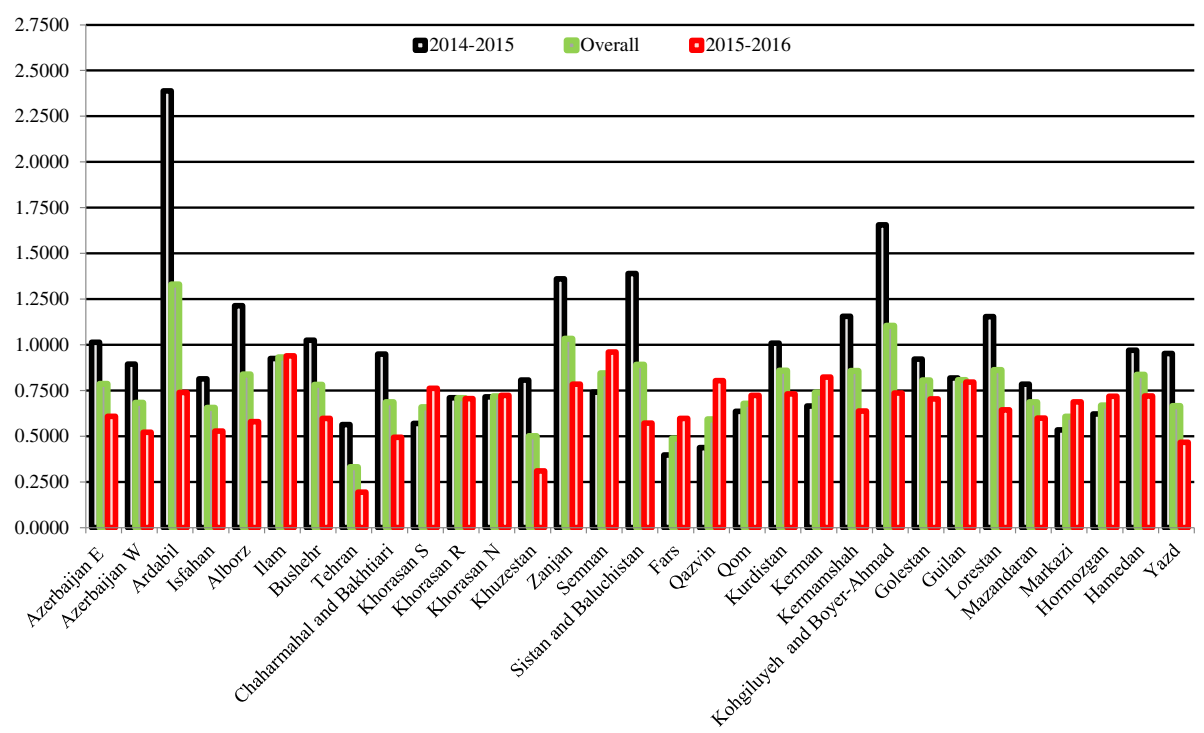

Fig. 6 The results of double-frontier MPI for the 31 Iranian provinces

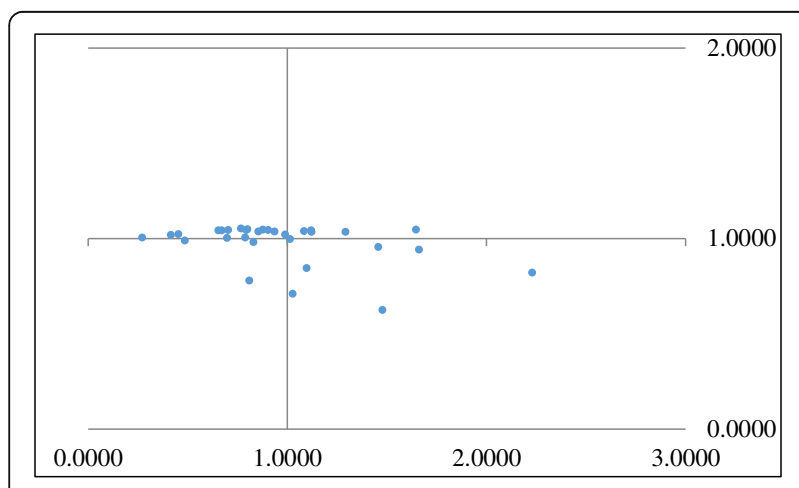

a) Optimistic technical and efficiency changes

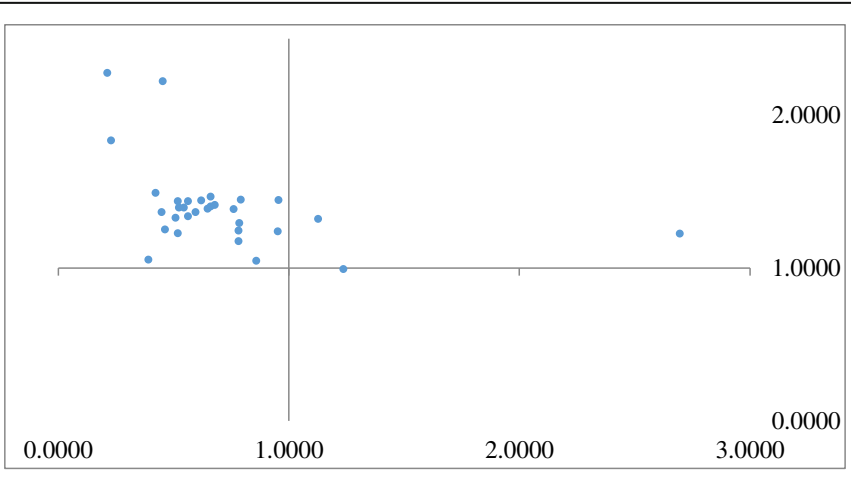

b) Pessimistic technical and efficiency changes

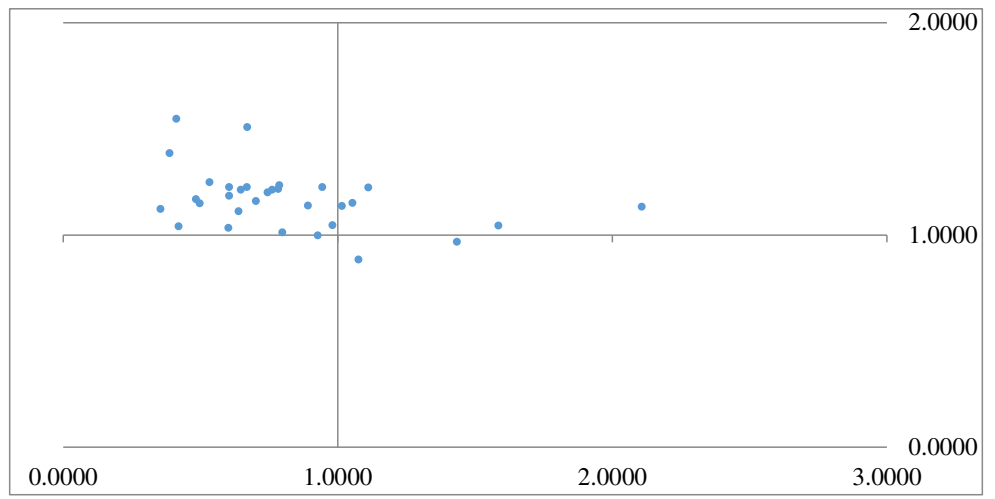

c) Aggregated technical and efficiency changes

Fig. 7 The results of negative or positive productivity growth in connection with technical and efficiency changes for the 31 Iranian provinces from 2014-2015. Vertical axes: efficiency change. Horizontal axes: technical change 


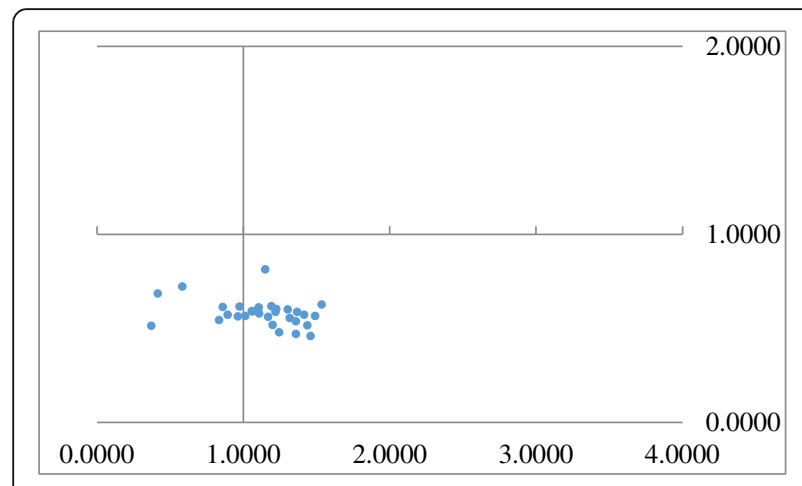

a) Optimistic technical and efficiency changes

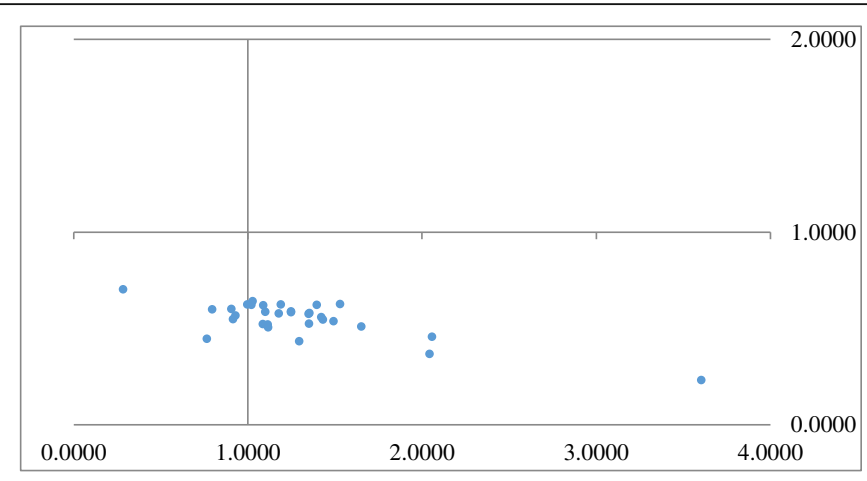

b) Pessimistic technical and efficiency changes

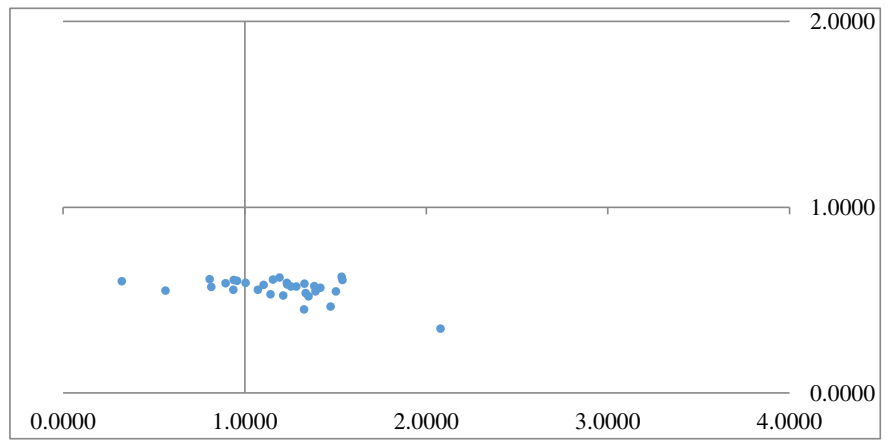

c) Aggregated technical and efficiency changes

Fig. 8 The results of negative or positive productivity growth in connection with technical and efficiency changes for the 31 Iranian provinces from 2015-2016. Vertical axes: efficiency change. Horizontal axes: technical change

the efficient frontier, but it can be equivalently measured by taking into account the anti-efficient frontier. To bridge this gap, the present study comprehensively examined Iranian road safety performance using a novel double frontier SBM model taking into account the efficient and anti-efficient frontiers simultaneously. The ER approach was then employed as a suitable method to aggregate the obtained results from both optimistic and pessimistic points of view. This is because of the fact that the additive independence condition may not be satisfied, since both the optimistic and pessimistic efficiency results are computed from the same data source.

To evaluate Iranian road safety performance, the input and output variables were selected based on policy relevance and data availability. For this purpose, the RMTO Statistical Yearbook was used as the main source of information. As a result, six input variables, including $P S$, $R M D, E \mathcal{E} V, C, E M S$, and $R L S$, and an output variable, $F R^{-1}$, were selected for assessing the road safety performance. Analysis of Iranian road safety performance using the proposed DF-SBM-ER illustrates that Alborz ranked $2^{\text {nd }}$ in the year 2014; meanwhile, it was the most efficient province in 2015 and 2016.
It is certainly crucial for the authorities to examine the strengths and weaknesses of their managerial decisions regarding each province. For this reason, the MPI has been recognized as an appropriate method for further analysis of each province in terms of road safety performance. Although the previous studies, more or less, utilized the optimistic MPI values for assessing DMUs, the current study carried out an in-depth analysis to assess Iranian provinces regarding road safety performance by investigating a novel DF-SBM-MPI, which simultaneously takes into account both efficiency changes and frontier shifts from the optimistic and pessimistic points of view over a period of time. The significant difference between the results obtained from the optimistic and pessimistic MPIs obviously confirms that taking account of the anti-efficient frontier is essential for a more comprehensive analysis of performance changes. The results will also assist the authorities to decide whether the strategic frontier shift is appropriate or not.

On average, the Iranian provinces' productivity in terms of road safety declined with a rate of $13.66 \%$ from 2014 to 2015, although the technology frontier shifted 


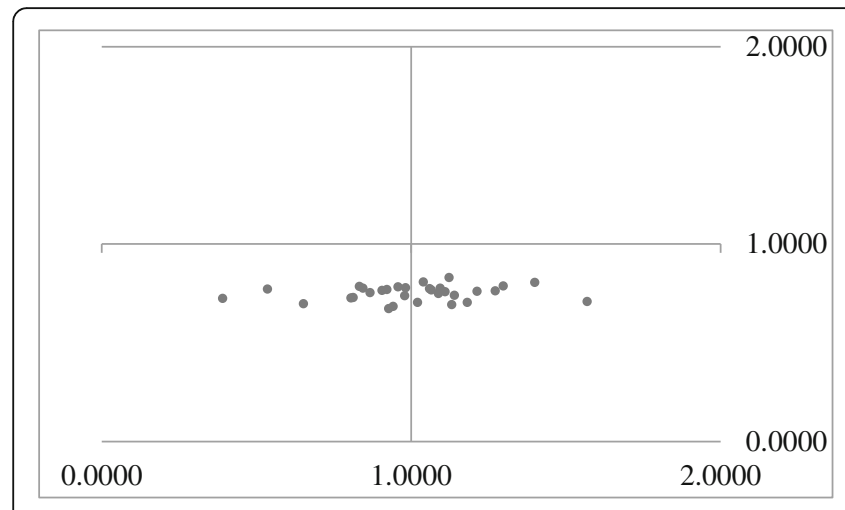

a) Optimistic technical and efficiency changes

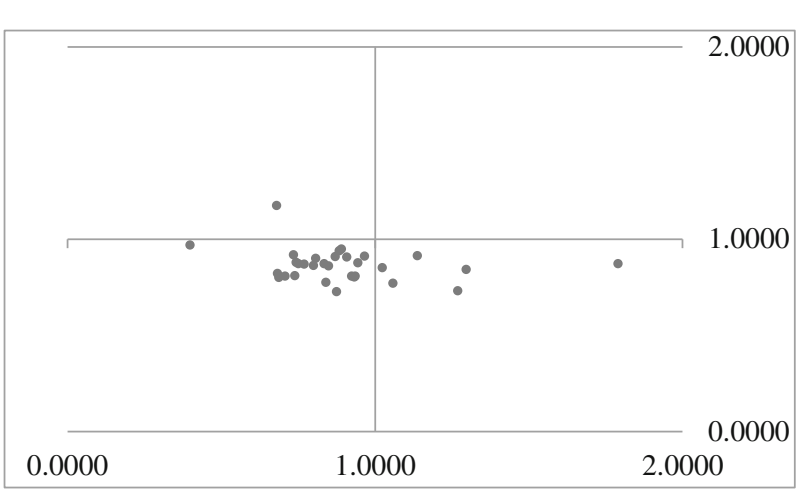

b) Pessimistic technical and efficiency changes

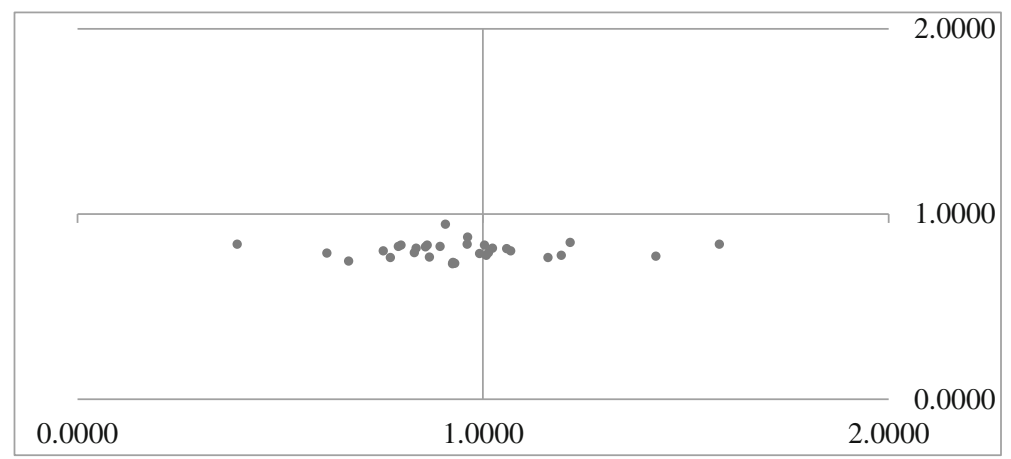

c) Aggregated technical and efficiency changes

Fig. 9 The results of negative or positive productivity growth in connection with technical and efficiency changes for the 31 Iranian provinces from 2014-2016. Vertical axes: efficiency change. Horizontal axes: technical change

with a positive rate of around $16 \%$. This means that the main reason for productivity reduction from 2014-2015 is the significant decline in provinces' efficiency, by an average of $25.54 \%$. On the contrary, the Iranian road safety performance technologically regressed, with an average reduction rate of $44 \%$ from 2015-2016; meanwhile, Iranian provinces experienced an improvement in efficiency changes, with an average reduction rate of $13 \%$. Generally speaking, the provinces' productivity in terms of road safety significantly decreased from 2015 to 2016, with a geometric mean rate of about $37 \%$.
Finally, taking into account the period 2014-2016, Iranian road safety declined with a mean rate of about $26 \%$, due to a reduction in efficiency changes (around $8 \%$ ) as well as a slight negative shift in technology frontier (about 19\%).

In brief, the conducted in-depth analysis reveals that Iranian provinces were successful in technical changes from 2014-2015, while from 2015-2016, they effectively improved their efficiencies, which is, of course, admirable. It is also worth mentioning that although the productivity of nine provinces on road safety was generally enhanced from 2014-2015; only five provinces fully

Table 10 Data analysis. The input and output slacks for Qom province

\begin{tabular}{|c|c|c|c|c|c|c|c|}
\hline \multirow[t]{2}{*}{ Years } & \multicolumn{6}{|l|}{$s_{i}^{-}$} & \multirow{2}{*}{$\begin{array}{l}S_{r}^{+} \\
F R^{-1}\end{array}$} \\
\hline & $\begin{array}{l}\text { Police } \\
\text { Station (PS) }\end{array}$ & $\begin{array}{l}\text { Road Maintenance } \\
\text { Depot (RMD) }\end{array}$ & $\begin{array}{l}\text { Equipment \& } \\
\text { Vehicles (E\&V) }\end{array}$ & Camera (C) & $\begin{array}{l}\text { Emergency Medical } \\
\text { Service (EMS) }\end{array}$ & $\begin{array}{l}\text { Road with Lighting } \\
\text { System (RLS) }\end{array}$ & \\
\hline 2014 & 0.1798190 & 0.02497436 & 11.87315 & 1.643356 & 0.0000 & 17.60741 & 0.6274015 \\
\hline 2015 & 0.2163227 & 0.1130736 & 14.54769 & 4.877088 & 0.0000 & 18.53491 & 0.9212618 \\
\hline 2016 & 0.1963451 & 0.1026324 & 11.06201 & 4.118692 & 0.0000 & 16.82283 & 0.3757682 \\
\hline
\end{tabular}


Table 11 Data analysis. The existing dataset for Qom province

\begin{tabular}{|c|c|c|c|c|c|c|c|}
\hline \multirow[t]{2}{*}{ Years } & \multicolumn{6}{|l|}{ Input(i) } & \multirow{2}{*}{$\begin{array}{l}\text { Output (r) } \\
F R^{-1}\end{array}$} \\
\hline & $\begin{array}{l}\text { Police } \\
\text { Station (PS) }\end{array}$ & $\begin{array}{l}\text { Road Maintenance } \\
\text { Depot (RMD) }\end{array}$ & $\begin{array}{l}\text { Equipment \& } \\
\text { Vehicles (E\&V) }\end{array}$ & Camera (C) & $\begin{array}{l}\text { Emergency Medical } \\
\text { Service (EMS) }\end{array}$ & $\begin{array}{l}\text { Road with Lighting } \\
\text { System (RLS) }\end{array}$ & \\
\hline 2014 & 0.55944 & 1.25874 & 26.01399 & 3.63636 & 2.65734 & 26.43357 & 2.13776 \\
\hline 2015 & 0.58997 & 1.32743 & 28.46608 & 6.93215 & 2.80236 & 27.87611 & 2.02564 \\
\hline 2016 & 0.53548 & 1.20482 & 21.15127 & 7.76439 & 2.54351 & 25.30120 & 1.40260 \\
\hline
\end{tabular}

progressed in road safety performance regarding both efficiency and technical changes.

It is suggested applying the proposed method in other aspects of transportation management. Using different nonlinear methods of aggregation rather than the ER algorithm would also be an interesting topic for future road safety research. In addition, it is suggested extending the method by using some weight restrictions on input variables. In this regard, some group decision-making methods can be applied to investigate the importance of each variable. Furthermore, proposing a double frontier Assurance Region SBM model can be of interest to the safety experts.

As mentioned earlier, Iranian safety experts suffer from lack of data. Road safety experts around the world are encouraged to conduct a research study based on the proposed method by defining some new input and output variables subject to data availability. They can define input variables such as "barriers to accidents", "traffic calming devices", "police officers or patrol units", "financial resources" (i.e. the amount of money spent on police patrols and highway maintenance) and "manpower"; meanwhile, "the number of seriously injured people" and "the number of road accidents" can also be used to define a new output variable.

As discussed in the literature, a number of studies have been carried out to assess the road safety performance of European countries using DEA models based only on the efficient frontier, which could not lead to a comprehensive assessment. The current study is the first attempt to assess road safety performance by considering

Table 12 Data analysis. Deviations of the input and output variables for Qom province

\begin{tabular}{|c|c|c|c|c|c|c|c|}
\hline \multirow[t]{2}{*}{ Years } & \multicolumn{6}{|l|}{ Input } & \multirow{2}{*}{$\begin{array}{l}\text { Output } \\
F R^{-1}\end{array}$} \\
\hline & $\begin{array}{l}\text { Police } \\
\text { Station (PS) }\end{array}$ & $\begin{array}{l}\text { Road Maintenance } \\
\text { Depot (RMD) }\end{array}$ & $\begin{array}{l}\text { Equipment \& } \\
\text { Vehicles (E\&V) }\end{array}$ & Camera (C) & $\begin{array}{l}\text { Emergency Medical } \\
\text { Service (EMS) }\end{array}$ & $\begin{array}{l}\text { Road with Lighting } \\
\text { System (RLS) }\end{array}$ & \\
\hline 2014 & 0.32143 & 0.01984 & 0.45641 & 0.45192 & 0.00000 & 0.66610 & 0.29349 \\
\hline 2015 & 0.36667 & 0.08518 & 0.51105 & 0.70355 & 0.00000 & 0.66490 & 0.45480 \\
\hline 2016 & 0.36667 & 0.08518 & 0.52300 & 0.53046 & 0.00000 & 0.66490 & 0.26791 \\
\hline
\end{tabular}

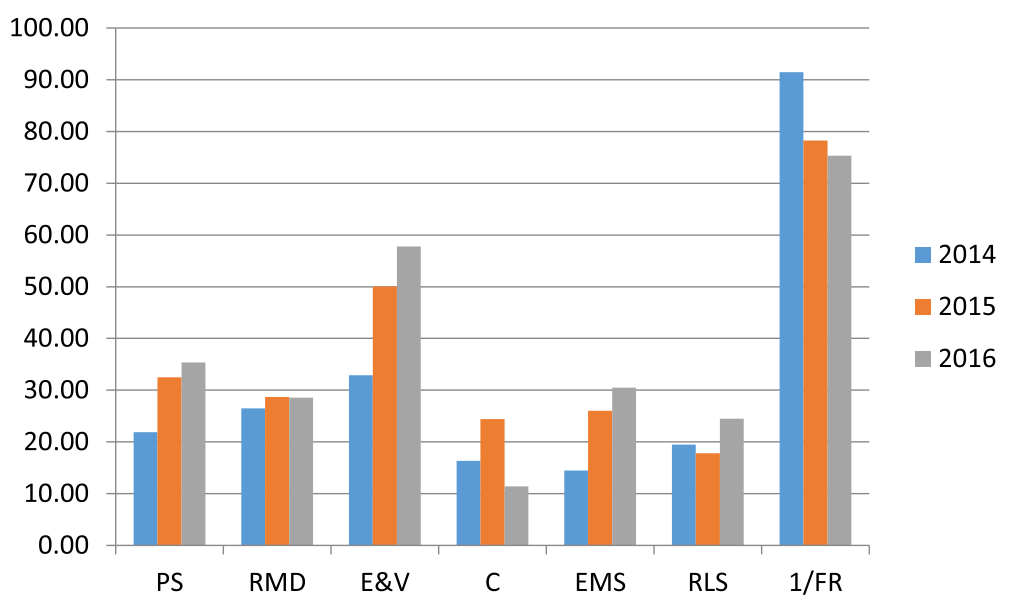

Fig. 10 The average deviation of input and output variables from the ideal situation for the years 2014-2016 


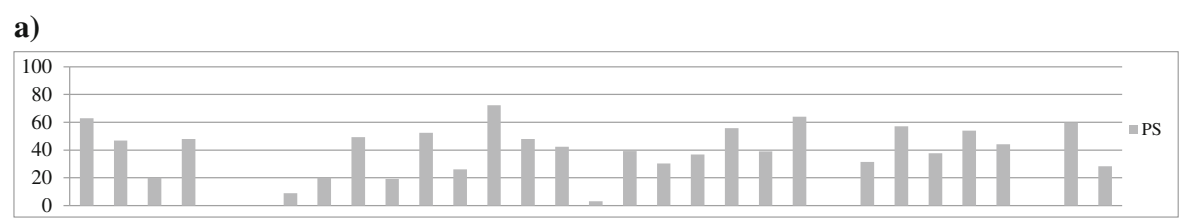

b)

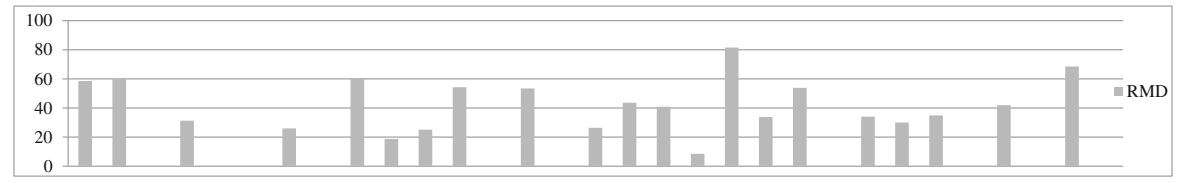

c)

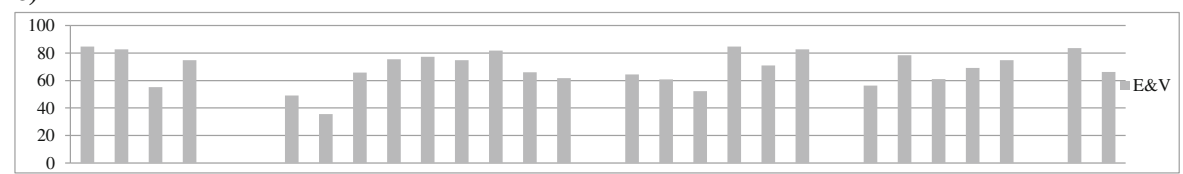

d)

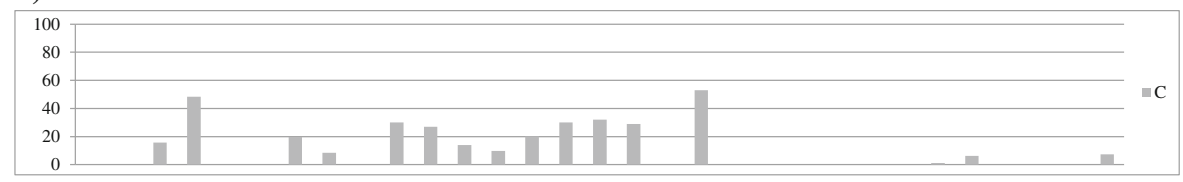

e)

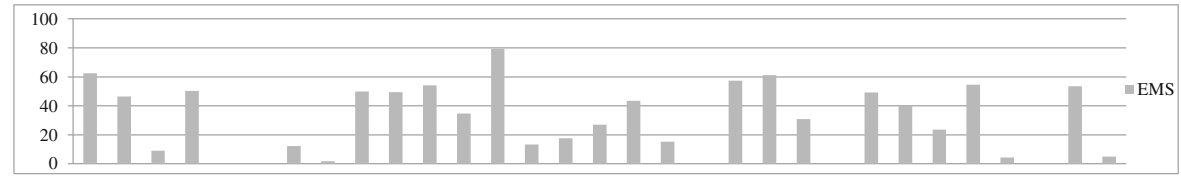

f)

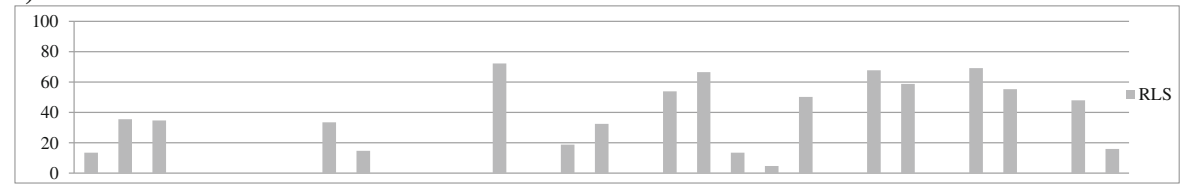

g)



Fig. 11 Deviations of input and output variables for Iranian provinces in 2016

the best-practice and the worst-practice frontiers. Since the focus of this study is on the basic characteristics of the proposed method, further applications are suggested as the future direction of the current research. The European countries can apply the proposed approach to assess their road safety performance more comprehensively. The input excesses and the output shortfalls obtained from the slack variables analysis can also provide the European authorities with a deeper insight into the road safety performance. In addition, the DF-SBM-ER can be further used by the European Commission to assess other aspects of transportation such as European rail transport, European air transport, and European maritime transport. 


\section{Appendix 1}

Suppose that there is an evaluation problem for assessing $n$ DMUs with $m$ inputs and $s$ outputs.

\subsection{1. The input-oriented CCR}

The optimistic input-oriented CCR model is mathematically as follows $[2,58]$ :

$$
\theta_{0}=\max \sum_{r=1}^{s} u_{r} y_{r 0}
$$

Subject to :

$$
\begin{aligned}
& \sum_{i=1}^{m} v_{i} x_{i 0}=1, \\
& \sum_{r=1}^{s} u_{r} y_{r j}-\sum_{i=1}^{m} v_{i} x_{i j} \leq 0, \quad j=1, \ldots, n \\
& u_{r}, v_{i} \geq 0, r=1, \ldots, s, \quad i=1, \ldots m
\end{aligned}
$$

where $u_{r}$ and $v_{i}$ denote the realtive weights of the outputs and the inputs, respectively. $x_{i j}$ and $y_{r j}$ also express the $i^{\text {th }}$ input and the $r^{\text {th }}$ output of $D M U_{j}$. $\theta_{0}$ denotes the efficiency degree of a given $D M U_{0}$ with the given inputs $\left(x_{i 0}\right)$ and the given outputs $\left(y_{r 0}\right)$.

The pessimistic or inverted input-oriented CCR model can be mathematically formulated as follows:

$$
\begin{aligned}
& \theta_{0}^{-1}=\min \sum_{r=1}^{s} u_{r} y_{r 0} \\
& \text { Subject to : } \\
& \sum_{i=1}^{m} v_{i} x_{i 0}=1, \\
& \sum_{r=1}^{s} u_{r} y_{r j}-\sum_{i=1}^{m} v_{i} x_{i j} \leq 0, \quad j=1, \ldots, n \\
& u_{r}, v_{i} \geq 0, r=1, \ldots, s, \quad i=1, \ldots m
\end{aligned}
$$

\subsection{2. The output-oriented CCR}

The optimistic output-oriented CCR model is mathematically as follows $[2,58]$ :

$$
\begin{aligned}
& \theta_{0}=\min \sum_{i=1}^{m} v_{i} x_{i 0} \\
& \text { Subject to: } \\
& \sum_{r=1}^{s} u_{r} y_{r 0}=1, \\
& \sum_{r=1}^{m} u_{r} y_{r j}-\sum_{i=1}^{m} v_{i} x_{i j} \leq 0, \quad j=1, \ldots, n \\
& u_{r}, v_{i} \geq 0, r=1, \ldots, s, \quad i=1, \ldots m
\end{aligned}
$$

where $u_{r}$ and $v_{i}$ denote the realtive weights of the outputs and the inputs, respectively. $x_{i j}$ and $y_{r j}$ also express the $i^{t h}$ input and the $r^{t h}$ output of $D M U_{j}$. $\theta_{0}$ denotes the efficiency degree of a given $D M U_{0}$ with the given inputs $\left(x_{i 0}\right)$ and the given outputs $\left(y_{r 0}\right)$.

The pessimistic or inverted output-oriented CCR model can be mathematically formulated as follows: $\theta_{0}^{-1}=\operatorname{mix} \sum_{i=1}^{m} v_{i} x_{i 0}$

Subject to :

$$
\begin{aligned}
& \sum_{r=1}^{s} u_{r} y_{r 0}=1, \\
& \sum_{r=1}^{s} u_{r} y_{r j}-\sum_{i=1}^{m} v_{i} x_{i j} \leq 0, \quad j=1, \ldots, n \\
& u_{r}, v_{i} \geq 0, r=1, \ldots, s, \quad i=1, \ldots m
\end{aligned}
$$

\section{Appendix 2}

Suppose that there is an evaluation problem for assessing $n$ DMUs with $m$ inputs and $s$ outputs.

\subsection{1. The optimistic SBM}

The optimistic SBM in time $t, D_{0}^{t}\left(x_{0}^{t}, y_{0}^{t}\right)$, can be mathematically represented as follows:

$$
D_{0}^{t}\left(x_{0}^{t}, y_{0}^{t}\right)=\operatorname{Min} \rho=q-\frac{1}{m} \sum_{i=1}^{m} S_{i}^{-} / x_{i 0}^{t}
$$

Subject to

$$
\begin{aligned}
& 1=q+(1 / s) \sum_{r=1}^{s} S_{r}^{+} / y_{r 0} \\
& q x_{i 0}^{t}=\sum_{j=1}^{n} x_{i j}^{t} \Lambda_{j}+S_{i}^{-}, \quad i=1, . ., m \\
& q y_{r 0}^{t}=\sum_{j=1}^{n} y_{r j}^{t} \Lambda_{j}-S_{r}^{+}, \quad r=1, . ., s \\
& \Lambda_{j} \geq 0, \quad S_{i}^{-} \geq 0, \quad S_{r}^{+} \geq 0, \quad q>0
\end{aligned}
$$

Similarly, the optimistic SBM in time $t+1, D_{0}^{t+1}\left(x_{0}^{t+1}\right.$, $\left.y_{0}^{t+1}\right)$, can be obtained by substituting $\left(x_{i 0}^{t+1}, x_{i j}^{t+1}, y_{r 0}^{t+1}, y_{r j}^{t+1}\right)$ with $\left(x_{i 0}^{t}, x_{i j}^{t}, y_{r 0}^{t}, y_{r j}^{t}\right)$ in model (36). As a result, $D_{0}^{t}\left(x_{0}^{t+1}\right.$, $\left.y_{0}^{t+1}\right)$, can be represented as follows:

$$
D_{0}^{t}\left(x_{0}^{t+1}, y_{0}^{t+1}\right)=\operatorname{Min} \rho=q-\frac{1}{m} \sum_{i=1}^{m} S_{i}^{-} / x_{i 0}^{t+1}
$$

Subject to

$$
\begin{aligned}
& 1=q+(1 / s) \sum_{r=1}^{s} S_{r}^{+} / y_{r 0} \\
& q x_{i 0}^{t+1}=\sum_{j=1}^{n} x_{i j}^{t} \Lambda_{j}+S_{i}^{-}, \quad i=1, . ., m \\
& q y_{r 0}^{t+1}=\sum_{j=1}^{n} y_{r j}^{t} \Lambda_{j}-S_{r}^{+}, \quad r=1, . ., s \\
& \Lambda_{j} \geq 0, \quad S_{i}^{-} \geq 0, \quad S_{r}^{+} \geq 0, \quad q>0
\end{aligned}
$$

Likewise, the second mixed period of measure, $D_{0}^{t+1}$ $\left(x_{0}^{t}, y_{0}^{t}\right)$, can be computed by substituting $\left(x_{i 0}^{t}, x_{i j}^{t+1}, y_{r 0}^{t}\right.$ ,$\left.y_{r j}^{t+1}\right)$ with $\left(x_{i 0}^{t+1}, x_{i j}^{t}, y_{r 0}^{t+1}, y_{r j}^{t}\right)$ in model (37). 


\subsection{2. The pessimistic point of view}

The pessimistic SBM in time $t, d_{0}^{t}\left(x_{0}^{t}, y_{0}^{t}\right)$, can be formulated as follows:

$$
d_{0}^{t}\left(x_{0}^{t}, y_{0}^{t}\right)=\operatorname{Max} \rho=q+\frac{1}{m} \sum_{i=1}^{m} S_{i}^{+} / x_{i 0}^{t}
$$

Subject to

$$
\begin{aligned}
& 1=q-(1 / s) \sum_{r=1}^{s} S_{r}^{-} / y_{r 0} \\
& q x_{i 0}^{t}=\sum_{j=1}^{n} x_{i j}^{t} \Lambda_{j}-S_{i}^{+} \text {, } \\
& q y_{r 0}^{t}=\sum_{j=1}^{n} y_{r j}^{t} \Lambda_{j}+S_{r}^{-} \text {, } \\
& \Lambda_{j} \geq 0, \quad S_{i}^{+} \geq 0, \quad S_{r}^{-} \geq 0, \quad q>0 \\
& i=1, . ., m \\
& r=1, . ., s
\end{aligned}
$$

By substituting $\left(x_{i 0}^{t+1}, x_{i j}^{t+1}, y_{r 0}^{t+1}, y_{r j}^{t+1}\right)$ with $\left(x_{i 0}^{t}, x_{i j}^{t}, y_{r 0}^{t}, y_{r j}^{t}\right)$ in model (38), the pessimistic SBM in time $t+1, d_{0}^{t+1}$ $\left(x_{0}^{t+1}, y_{0}^{t+1}\right)$, can be computed. Moreover, the first mixed period measure, $d_{0}^{t}\left(x_{0}^{t+1}, y_{0}^{t+1}\right)$, can be formulated as follows:

$$
d_{0}^{t}\left(x_{0}^{t+1}, y_{0}^{t+1}\right)=\operatorname{Min} \rho=q+\frac{1}{m} \sum_{i=1}^{m} S_{i}^{+} / x_{i 0}^{t+1}
$$

Subject to

$$
\begin{array}{lll}
1=q+(1 / s) \sum_{r=1}^{s} S_{r}^{-} / y_{r 0} & & \\
q x_{i 0}^{t+1}=\sum_{j=1}^{n} x_{i j}^{t} \Lambda_{j}-S_{i}^{+}, & & i=1, . ., m \\
q y_{r 0}^{t+1}=\sum_{j=1}^{n} y_{r j}^{t} \Lambda_{j}+S_{r}^{-}, & & r=1, . ., s \\
\Lambda_{j} \geq 0, \quad S_{i}^{+} \geq 0, \quad S_{r}^{-} \geq 0, & q>0 &
\end{array}
$$

Likewise, the second mixed period of measure, $d_{0}^{t+1}$ $\left(x_{0}^{t}, y_{0}^{t}\right)$, can be computed by substituting $\left(x_{i 0}^{t}, x_{i j}^{t+1}, y_{r 0}^{t}\right.$ ,$\left.y_{r j}^{t+1}\right)$ with $\left(x_{i 0}^{t+1}, x_{i j}^{t}, y_{r 0}^{t+1}, y_{r j}^{t}\right)$ in model (39).

\section{Appendix 3}

\subsection{1. The optimistic Super-SBM [49]}

Suppose that DMU $\left(\boldsymbol{x}_{\mathbf{0}}, \boldsymbol{y}_{\mathbf{0}}\right)$ is efficient. The production possibility set is defined as follows:

$$
P \backslash\left(x_{0}, y_{0}\right)=\left\{(\bar{x}, \bar{y}) \mid \bar{x} \geq \sum_{j=1 \neq 0}^{n} \lambda_{j} x_{j}, \bar{y} \leq \sum_{j=1 \neq 0}^{n} \lambda_{j} y_{j}, \bar{y} \geq 0, \lambda_{j} \geq 0\right\}
$$

$\bar{P} \backslash\left(x_{0}, y_{0}\right)$ is further defined as a subset of $P \backslash\left(x_{0}, y_{0}\right)$ :

$$
\bar{P} \backslash\left(x_{0}, y_{0}\right)=P \backslash\left(x_{0}, y_{0}\right) \cap\left\{\bar{x} \geq x_{0} \text { and } \bar{y} \leq y_{0}\right\}
$$

The optimistic Super-SBM is mathematically represented as follows:

$$
\delta^{*}=\operatorname{Min} \delta=\frac{\frac{1}{m} \sum_{i=1}^{m} \bar{x}_{i} / x_{i 0}}{\frac{1}{r} \sum_{r=1}^{s} \bar{y}_{r} / y_{r 0}}
$$

Subject to

$$
\begin{array}{lll}
\bar{x}_{i} \geq \sum_{j=1 \neq 0}^{n} \lambda_{j} x_{i j}, & i=1, . ., m \\
\bar{y}_{r} \leq \sum_{j=1 \neq 0}^{n} \lambda_{j} y_{r j}, & r=1, . ., s \\
\bar{x}_{i} \geq x_{i 0}, & \bar{y}_{r} \leq y_{r 0} & \\
\bar{y}_{r} \geq 0, & \lambda_{j} \geq 0 &
\end{array}
$$

Using Charnes-Cooper transformation, the fractional programming model (42) is transformed into the following linear programming model:

$$
\tau^{*}=\operatorname{Min} \tau=\frac{1}{m} \sum_{i=1}^{m} \tilde{x}_{i} / x_{i 0}
$$

Subject to

$$
\begin{array}{lrr}
1=\frac{1}{s} \sum_{r=1}^{s} \tilde{y}_{r} / y_{r 0}, & \\
\tilde{x}_{i} \geq \sum_{j=1 \neq 0}^{n} \Lambda_{j} x_{i j}, & i=1, . ., m \\
\tilde{y}_{r} \leq \sum_{j=1 \neq 0}^{n} \Lambda_{j} y_{r j}, & r=1, . ., s \\
\tilde{x}_{i} \geq q x_{i 0}, & \tilde{y}_{r} \leq q y_{r} & \\
\tilde{y}_{r} \geq 0, & \Lambda_{j} \geq 0 & q \geq 0
\end{array}
$$

The optimal solution of model (43) is $\left(\tau^{*}, \tilde{x}_{i}^{*}, \tilde{y}_{r}^{*}, \Lambda_{j}^{*}, q^{*}\right)$. Subsequently, the optimal solution of Super-SBM is $\left(\delta^{*}=\tau^{*}, \lambda_{j}^{*}=\Lambda_{j}^{*} / q^{*}, \bar{x}_{i}^{*}=\tilde{x}_{i}^{*} / q^{*}, \bar{y}_{r}^{*}=\tilde{y}_{r}^{*} / q^{*}\right)$.

\subsection{2. The pessimistic Super-SBM}

The production possibility set can be defined as follows:

$$
P \backslash\left(x_{0}, y_{0}\right)=\left\{(\bar{x}, \bar{y}) \mid \bar{x} \leq \sum_{j=1 \neq 0}^{n} \lambda_{j} x_{j}, \bar{y} \geq \sum_{j=1 \neq 0}^{n} \lambda_{j} y_{j}, \bar{x} \geq 0, \lambda_{j} \geq 0\right\}
$$

Furthermore, $\bar{P} \backslash\left(x_{0}, y_{0}\right)$ can be defined as a subset of $\left(x_{0}, y_{0}\right)$ :

$$
\bar{P} \backslash\left(x_{0}, y_{0}\right)=P \backslash\left(x_{0}, y_{0}\right) \cap\left\{\bar{x} \leq x_{0} \text { and } \bar{y} \geq y_{0}\right\}
$$

The pessimistic Super-SBM can be formulated as follows: 


$$
\delta^{*}=\operatorname{Max} \delta=\frac{\frac{1}{m} \sum_{i=1}^{m} \bar{x}_{i} / x_{i 0}}{\frac{1}{s} \sum_{r=1}^{s} \bar{y}_{r} / y_{r 0}}
$$

Subject to

$$
\begin{aligned}
& \bar{x}_{i} \leq \sum_{j=1 \neq 0}^{n} \lambda_{j} x_{i j}, \\
& i=1, . ., m \\
& \bar{y}_{r} \geq \sum_{j=1 \neq 0}^{n} \lambda_{j} y_{r j}, \\
& r=1, . ., s \\
& \bar{x}_{i} \leq x_{i 0} \text {, } \\
& \bar{y}_{r} \geq y_{r 0} \\
& \bar{x}_{i} \geq 0 \text {, } \\
& \lambda_{j} \geq 0
\end{aligned}
$$

Subsequently, the following linear programming model can be pessimistically obtained using the CharnesCooper transformation technique:

$$
\tau^{*}=\operatorname{Max} \tau=\frac{1}{m} \sum_{i=1}^{m} \tilde{x}_{i} / x_{i 0}
$$

Subject to

$$
\begin{aligned}
& 1=\frac{1}{s} \sum_{r=1}^{s} \tilde{y}_{r} / y_{r 0}, \\
& \tilde{x}_{i} \leq \sum_{j=1 \neq 0}^{n} \Lambda_{j} x_{i j}, \quad i=1, . ., m \\
& \tilde{y}_{r} \geq \sum_{j=1 \neq 0}^{n} \Lambda_{j} y_{r j}, \quad r=1, . ., s \\
& \tilde{x}_{i} \leq q x_{i 0}, \quad \tilde{y}_{r} \geq q y_{r 0} \\
& \tilde{x}_{i} \geq 0, \quad \Lambda_{j} \geq 0 \quad q \geq 0
\end{aligned}
$$

The optimal solution of (47) is $\left(\tau^{*}, \tilde{x}_{i}^{*}, \tilde{y}_{r}^{*}, \Lambda_{j}^{*}, q^{*}\right)$. Subsequently, the optimal solution of Super-SBM is $\left(\delta^{*}=\tau^{*}, \lambda_{j}^{*}\right.$ $\left.=\Lambda_{j}^{*} / q^{*}, \bar{x}_{i}^{*}=\tilde{x}_{i}^{*} / q^{*}, \bar{y}_{r}^{*}=\tilde{y}_{r}^{*} / q^{*}\right)$.

\section{Appendix 4}

\subsection{1. The optimistic point of view}

In the situation that $D_{0}^{t}\left(x_{0}^{t}, y_{0}^{t}\right)=1, D M U_{0}$ is efficient based on the data set belonging to time period $t$. Therefore, the super efficiency score, $\tilde{D}_{0}^{t}\left(x_{0}^{t}, y_{0}^{t}\right)$, is optimistically measured as follows:

$$
\begin{aligned}
& \tilde{D}_{0}^{t}\left(x_{0}^{t}, y_{0}^{t}\right)=\operatorname{Min} \tau=\frac{1}{m} \sum_{i=1}^{m} \tilde{x}_{i}^{t} / x_{i 0}^{t} \\
& \text { Subject to } \\
& 1=\frac{1}{s} \sum_{r=1}^{s} \tilde{y}_{r}^{t} / y_{r 0}^{t}, \\
& \tilde{x}_{i}^{t} \geq \sum_{j=1 \neq 0}^{n} \Lambda_{j} x_{i j}^{t}, \quad i=1, . ., m \\
& \tilde{y}_{r}^{t} \leq \sum_{j=1 \neq 0}^{n} \Lambda_{j} y_{r j}^{t}, \quad r=1, . ., s \\
& \tilde{x}_{i}^{t} \geq q x_{i 0}^{t} \text {, } \\
& \tilde{y}_{r}^{t} \leq q y_{r 0}^{t} \\
& \tilde{y}_{r}^{t} \geq 0, \quad \Lambda_{j} \geq 0 \quad q \geq 0
\end{aligned}
$$

By substituting $\left(x_{i 0}^{t+1}, \tilde{x}_{i}^{t+1}, x_{i j}^{t+1}, y_{r 0}^{t+1}, \tilde{y}_{r}^{t+1}, y_{r j}^{t+1}\right)$ with $\left(x_{i 0}^{t}, \tilde{x}_{i}^{t}, x_{i j}^{t}, y_{r 0}^{t}, \tilde{y}_{r}^{t}, y_{r j}^{t}\right)$ in model (48), the super efficiency in time period $t+1, \tilde{D}_{0}^{t+1}\left(x_{0}^{t+1}, y_{0}^{t+1}\right)$, can be optimistically computed. Moreover, the first mixed period of measure, $\tilde{D}_{0}^{t}\left(x_{0}^{t+1}, y_{0}^{t+1}\right)$, can be formulated as follows:

$$
\tilde{D}_{0}^{t}\left(x_{0}^{t+1}, y_{0}^{t+1}\right)=\operatorname{Min} \tau=\frac{1}{m} \sum_{i=1}^{m} \tilde{x}_{i}^{t+1} / x_{i 0}^{t+1}
$$

Subject to

$$
\begin{aligned}
& 1=\frac{1}{s} \sum_{r=1}^{s} \tilde{y}_{r}^{t+1} / y_{r 0}^{t+1}, \\
& \tilde{x}_{i}^{t+1} \geq \sum_{j=1 \neq 0}^{n} \Lambda_{j} x_{i j}^{t}, \quad i=1, . ., m \\
& \tilde{y}_{r}^{t+1} \leq \sum_{j=1 \neq 0}^{n} \Lambda_{j} y_{r j}^{t}, \quad r=1, . ., s \\
& \tilde{x}_{i}^{t+1} \geq q x_{i 0}^{t+1}, \\
& \tilde{y}_{r}^{t+1} \leq q y_{r 0}^{t+1} \\
& \tilde{y}_{r}^{t+1} \geq 0, \quad \Lambda_{j} \geq 0 \quad q \geq 0
\end{aligned}
$$

Likewise, the second mixed period of measure, $D_{0}^{t+1}$ $\left(x_{0}^{t}, y_{0}^{t}\right)$, can be computed by substituting $\left(x_{i 0}^{t}, \tilde{x}_{i}^{t}, x_{i j}^{t+1}\right.$, $\left.y_{r 0}^{t}, \tilde{y}_{r}^{t}, y_{r j}^{t+1}\right) \quad$ with $\quad\left(x_{i 0}^{t+1}, \tilde{x}_{i}^{t+1}, x_{i j}^{t}, y_{r 0}^{t+1}, \tilde{y}_{r}^{t+1}, y_{r j}^{t}\right) \quad$ in model (49).

\subsection{2. The pessimistic point of view}

In the situation that $d_{0}^{t}\left(x_{0}^{t}, y_{0}^{t}\right)=1$ which means that $D M U_{0}$ is efficient based on the data set belongs to time period $t$. Therefore, the super efficiency score, $\tilde{d}_{0}^{t}\left(x_{0}^{t}, y_{0}^{t}\right)$, is measured from the pessimistic point of view as follows:

$$
\tilde{d}_{0}^{t}\left(x_{0}^{t}, y_{0}^{t}\right)=\max \tau=\frac{1}{m} \sum_{i=1}^{m} \tilde{x}_{i}^{t} / x_{i 0}^{t}
$$

Subject to

$$
\begin{array}{ll}
1=\frac{1}{s} \sum_{r=1}^{s} \tilde{y}_{r}^{t} / y_{r 0}^{t}, & \\
\tilde{x}_{i}^{t} \leq \sum_{j=1 \neq 0}^{n} \Lambda_{j} x_{i j}^{t}, & i=1, . ., m \\
\tilde{y}_{r}^{t} \geq \sum_{j=1 \neq 0}^{n} \Lambda_{j} y_{r j}^{t}, & r=1, . ., s \\
\tilde{x}_{i}^{t} \leq q x_{i 0}^{t}, & \\
\tilde{y}_{r}^{t} \geq q y_{r 0}^{t} & \\
\tilde{x}_{i}^{t} \geq 0, \quad \Lambda_{j} \geq 0 \quad q \geq 0 &
\end{array}
$$

By substituting $\left(x_{i 0}^{t+1}, \tilde{x}_{i}^{t+1}, x_{i j}^{t+1}, y_{r 0}^{t+1}, \tilde{y}_{r}^{t+1}, y_{r j}^{t+1}\right)$ with $\left(x_{i 0}^{t}, \tilde{x}_{i}^{t}, x_{i j}^{t}, y_{r 0}^{t}, \tilde{y}_{r}^{t}, y_{r j}^{t}\right)$ in model (50), the super efficiency in time period $t+1, \tilde{d}_{0}^{t+1}\left(x_{0}^{t+1}, y_{0}^{t+1}\right)$ can be pessimistically 
computed. Moreover, the first mixed period of measure, $\tilde{d}_{0}^{t}\left(x_{0}^{t+1}, y_{0}^{t+1}\right)$, can be formulated as follows:

$$
\tilde{d}_{0}^{t}\left(x_{0}^{t+1}, y_{0}^{t+1}\right)=\max \tau=\frac{1}{m} \sum_{i=1}^{m} \tilde{x}_{i}^{t+1} / x_{i 0}^{t+1}
$$

\section{Subject to}

$$
\begin{aligned}
& 1=\frac{1}{s} \sum_{r=1}^{s} \tilde{y}_{r}^{t+1} / y_{r 0}^{t+1}, \\
& \tilde{x}_{i}^{t+1} \leq \sum_{j=1 \neq 0}^{n} \Lambda_{j} x_{i j}^{t}, \quad i=1, . ., m \\
& \tilde{y}_{r}^{t+1} \geq \sum_{j=1 \neq 0}^{n} \Lambda_{j} y_{r j}^{t}, \quad r=1, . ., s \\
& \tilde{x}_{i}^{t+1} \leq q x_{i 0}^{t+1}, \\
& \tilde{y}_{r}^{t+1} \geq q y_{r 0}^{t+1} \\
& \tilde{x}_{i}^{t+1} \geq 0, \quad \Lambda_{j} \geq 0 \quad q \geq 0
\end{aligned}
$$

Likewise, the second mixed period of measure, $d_{0}^{t+1}$ $\left(x_{0}^{t}, y_{0}^{t}\right)$, can be computed by substituting $\left(x_{i 0}^{t}, \tilde{x}_{i}^{t}, x_{i j}^{t+1}, y_{r 0}^{t}\right.$, $\left.\tilde{y}_{r}^{t}, y_{r j}^{t+1}\right)$ with $\left(x_{i 0}^{t+1}, \tilde{x}_{i}^{t+1}, x_{i j}^{t}, y_{r 0}^{t+1}, \tilde{y}_{r}^{t+1}, y_{r j}^{t}\right)$ in model (51).

\begin{abstract}
Abbreviations
DEA : Data Envelopment Analysis; CCR : Charnes, Cooper and Rhodes; SBM : Slacks-Based Measure; DMU: Decision Making Unit; ER: Evidential Reasoning; DF-SBM- MPI: Double-Frontier SBM-based Malmquist Productivity Index; MPI: Malmquist Productivity Index; WHO: World Health Organization; DFSBM-ER: Double-Frontier SBM aggregated by ER algorithm; BCC: Banker, Charnes and Cooper; DEA-RS : DEA-based Road Safety model; TOPSIS: Technique for Order Preference by Similarity to an Ideal Solution; PMPI : Pessimistic SBM-based MPI; OMPI: Optimistic SBM-based MPI; RMTO: Road Maintenance and Transportation Organization; PS: Police Station; RMD : Road Maintenance Depot; E\&V: Equipment and vehicles; C: Camera; EMS : Emergency medical service; RLS: Road with lighting system
\end{abstract}

\section{Acknowledgements}

Not applicable

\section{Funding}

Not applicable

\section{Availability of data and materials}

The required data were collected from the following website (in Persian) http://www.rmto.ir/Pages/SalnameAmari.aspx

\section{Authors' contributions}

Both authors read and approved the final manuscript.

\section{Competing interests}

The authors declare that they have no competing interests.

\section{Publisher's Note}

Springer Nature remains neutral with regard to jurisdictional claims in published maps and institutional affiliations.

Received: 17 August 2018 Accepted: 4 December 2018

Published online: 16 January 2019

\section{References}

1. World Health Organization. 2015. Global status report on road safety. http:// apps.who.int/iris/bitstream/10665/44122/1/9789241563840_eng.pdf

2. Charnes, A., Cooper, W. W., \& Rhodes, E. (1978). Measuring the efficiency of decision making units. European Journal of Operational Research, 2, 429-444.
3. Odeck, J. (2000). Assessing the relative efficiency and productivity growth of vehicle inspection services: an application of DEA and Malmquist indices. European Journal of Operational Research, 126, 501-514.

4. Odeck, J. (2006). Identifying traffic safety best practice: An application of DEA and Malmquist indices. Omega, 34(1), 28-40.

5. Hermans, E., Van den Bossche, F., \& Wets, G. (2008). Combining road safety information in a performance index. Accident Analysis and Prevention, 40, 1337-1344.

6. Hermans, E., Brijs, T., Wets, G., \& Vanhoof, K. (2009). Benchmarking road safety: Lessons to learn from a data envelopment analysis. Accident Analysis and Prevention, 41, 174-182.

7. Shen, Y., Hermans, E., Ruan, D., Wets, G., Brijs, T., \& Vanhoof, K. (2011). A generalized multiple layer data envelopment analysis model for hierarchical structure assessment: A case study in road safety performance evaluation. Expert Systems with Applications, 38, 15262-15272.

8. Shen, Y., Hermans, E., Brijs, T., Wets, G., \& Vanhoof, K. (2012). Road safety risk evaluation and target setting using data envelopment analysis and its extensions. Accident Analysis and Prevention, 48, 430-441.

9. Shen, Y., Hermans, E., Bao, Q., Brijs, T., \& Wets, G. (2013). Road safety development in Europe: A decade of changes (2001-2010). Accident Analysis and Prevention, 60, 85-94.

10. Egilmez, G., \& McAvoy, D. (2013). Benchmarking road safety of U.S. states: A DEA-based Malmquist productivity index approach. Accident Analysis and Prevention, 53, 55-64.

11. Shen, Y., Hermans, E., Bao, Q., Brijs, T., \& Wets, G. (2015). Serious Injuries: An Additional Indicator to Fatalities for Road Safety Benchmarking. Traffic Injury Prevention, 16, 246-253.

12. Bastos, J. T., Shen, Y., Hermans, E., Brijs, T., Wets, G., \& Ferraz, A. C. P. (2015). Traffic fatality indicators in Brazil: State diagnosis based on data envelopment analysis research. Accident Analysis and Prevention 81, 61-73.

13. Rosic, M., Pesic, D., Kukic, D., Antic, B., \& Bozovic, M. (2017). Method for selection of optimal road safety composite index with examples from DEA and TOPSIS method. Accident Analysis and Prevention, 98, 277-286.

14. Behnood, H. R., Ayati, E., Hermans, E., \& Pirayesh Neghab, M. A. (2014). The Road safety performance evaluation and policy making by data envelopment analysis: A case study of provincial data in Iran. Scientia Iranica A, 21(5), 1515-1528.

15. Ganji, S. S., \& Rassafi, A. A. (2018). Measuring the road traffic safety performance of Iranian provinces: A double-frontier DEA model and evidential reasoning approach. International Journal of Injury Control and Safety Promotion In press.

16. Charnes, A., Cooper, W. W., Golany, B., Seiford, L., \& Stutz, J. (1985). Foundations of Data Envelopment Analysis for Pareto-Koopmans Efficient Empirical Production Functions. Journal of Econometrics, 30(1/2), 91-107.

17. Tone, K. (2001). A slack-based measure of efficiency in data envelopment analysis. European Journal of Operational Research, 130(3), 498-509.

18. Yang, G.-L., Yang, J.-B., Liu, W.-B., \& Li, X.-X. (2013). Cross-efficiency aggregation in DEA models using the evidential-reasoning approach. European Journal of Operational Research, 231(2), 393-404.

19. Cao, J., Chen, G., Khoveyni, M., Eslami, R., \& Yang, G.-L. (2016). Specification of a performance indicator using the Evidential-Reasoning approach. Knowledge-Based Systems, 92, 138-150.

20. Banker, R. D., Charnes, A., \& Cooper, W. W. (1984). Some models for estimating technical and scale efficiencies in data envelopment analysis. Management Science, 30(9), 1078-1092.

21. Cherchye, L., Moesen, W., Rogge, N., Van Puyenbroeck, T., Saisana, M., Saltelli, A., Liska, R., \& Tarantola, S. (2008). Creating composite indicators with DEA and robustness analysis: the case of the Technology Achievement Index. Journal of the Operational Research Society, 59, 239-251.

22. Azizi, H., \& Fathi Ajirlu, S. (2010). Measurement of overall performances of decision-making units using ideal and anti-ideal decision-making units. Computers and Industrial Engineering, 59, 411-418.

23. Azizi, H. (2011). The interval efficiency based on the optimistic and pessimistic points of view. Applied Mathematical Modelling, 35(5), 2384-2393

24. Azizi, H. (2014). DEA efficiency analysis: A DEA approach with double frontiers. International Journal of Systems Science, 45(11), 2289-2230.

25. Yang, J. B., \& Singh, M. G. (1994). An Evidential Reasoning Approach for multiple attribute decision making with uncertainty. IEEE Transactions on Systems, Man, and Cybernetics, 24(1), 1-18. 
26. Dempster, A. P. (1967). Upper and lower probabilities induced by a multivalued mapping. Annals of Mathematical Statistics, 38(2), 325-339.

27. Shafer, G. (1976). A mathematical theory of evidence. Princeton University Press, Princeton, NJ.

28. Zadeh, L. A. (1984). Review of books: A mathematical theory of evidence. Al Magazine, 5(3), 81-83.

29. Buchanan, B. G., \& Shortliffe, E. H. (1984). Rule-based expert system. Reading: Addison-Wesley,

30. Murphy, C. K. (2000). Combining belief functions when evidence conflicts. Decision Support System, 29, 1-9.

31. Yang, J. B., \& Sen, P. (1994). A general multi-level evaluation process for hybrid MADM with uncertainty. IEEE Transactions on Systems, Man, and Cybernetics, 24(10), 1458-1473.

32. Yang, J. B., \& Xu, D. L. (2002). On the evidential reasoning algorithm for multiple attribute decision analysis under uncertainty. IEEE Transactions on Systems, Man, and Cybernetics-Part A, 32(2), 289-304.

33. Ganji, S. S., \& Rassafi, A. A. (2019). Road Safety Evaluation using a Novel Cross Efficiency Method based on Double Frontiers DEA and Evidential Reasoning Approach. KSCE Journal of Civil Engineering, In press.

34. Xu, D. L., \& Yang, J. B. (2005). An intelligent decision system based on the evidential reasoning approach and its applications. Journal of Telecommunications and Information Technology, 3, 73-80.

35. Xu, D. L. (2009). Assessment of nuclear waste repository options using the ER approach. International Journal of Information Technology and Decision Making, 8(3), 581.

36. Xu, D. L. (2012). An introduction and survey of the evidential reasoning approach for multiple criteria decision analysis. Annals of Operations Research, 195, 163-187.

37. Yang, J. B., \& Xu, D. L. (2013). Evidential reasoning rule for evidence combination. Artificial Intelligence, 205, 1-29.

38. Yang, J. B. and Xu, D. L. 2014. A study on generalizing Bayesian inference to evidential reasoning, in Belief Function: Theory and Applications, Lecture Notes in Artificial Intelligence, Fabio Cuzzolin (ed.), Springer, ISBN 978-3-31911191-9, 180-189.

39. Rassafi, A. A., Ganji, S. S., \& Purkhani, H. (2018). Road safety assessment under uncertainty using a Multi Attribute Decision Analysis based on DempsterShafer theory. KSCE Journal of Civil Engineering, 22(8), 3137-3152.

40. Ganji, S. S., Rassafi, A. A., \& Kordani, A. A. (2018). Vehicle safety analysis based on a hybrid approach integrating DEMATEL, ANP and ER. KSCE Journal of Civil Engineering, 22(11), 4580-4592.

41. Malmquist, S. (1953). Index numbers and indifference surfaces. Trabajos de Estatistica, 4, 209-242.

42. Färe, R., Grosskopf, S., Lindgren, B., \& Roos, P. (1992). Productivity change in Swedish pharmacies 1980-1989: a nonparametric Malmquist approach. Journal of Productivity Analysis, 3, 85-102.

43. Caves, D. W., Christensen, L. R., \& Diewert, W. E. (1982). The economic theory of index numbers and the measurement of input, output, and productivity. Econometrica, 50(6), 1393-1414

44. Farrell, M. J. (1957). The measurement of productivity efficiency. Journal of the Royal Statistical Society Series A: General, 120(3), 253-281.

45. Chen, Y., \& Agha Ighbal, A. (2004). DEA Malmquist productivity measure: New insights with an application to computer industry. European Journal of Operational Research, 159, 239-249.

46. Al-Eraqi, A. S., Khader, A. T., \& Mustafa, A. (2009). DEA Mamquist index measurement in Middle East and East African containers terminals. International Journal of Shipping and Transport Logistics, 1(3), 249-259.

47. Wang, Y.-M., \& Lan, Y.-X. (2011). Measuring Malmquist productivity index: A new approach based on double frontiers data envelopment analysis. Mathematical and Computer Modelling, 54, 2760-2771.

48. Liu, F.-H. F., \& Wang, P.-H. (2008). DEA Malmquist productivity measure: Taiwanese semiconductor companies. International Journal of Production Economics, 112, 367-379.

49. Tone, K. (2002). A slack-based measure of super-efficiency in data envelopment analysis. European Journal of Operational Research, 143(1), 32-41.

50. Liu, F.-H. F., \& Chen, C.-L. (2009). The worst-Practice DEA model with slackbased measurement. Computers and Industrial Engineering, 57, 496-505.

51. SafetyNet, Work Package 3. (2005). State-of-the-art Report on Road Safety Performance Indicators. In Stichting Wetenschappelijk Onderzoek verkeersveiligheid.

52. European Commission (EC). 2010 b. EU Energy and Transport in Figures 2010. Commission of the European Communities, Brussels.
53. European Commission (EC). 2012. EU Energy and Transport in Figures 2012. Commission of the European Communities, Brussels.

54. Research and Innovative Technology Administration (RITA). 2010. State Transportation Statistics. State Transportation Statistics.

55. Road Maintenance and Transportation Organization (RMTO). (2014). The Statistical Yearbook, Ministry of Road and Urban Development. In Iran.

56. Road Maintenance and Transportation Organization (RMTO). (2015). The Statistical Yearbook, Ministry of Road and Urban Development. In Iran.

57. Road Maintenance and Transportation Organization (RMTO). (2016). The Statistical Yearbook, Ministry of Road and Urban Development. In Iran.

58. Cooper, W. W., Seiford, L. M., \& Tone, K. (2007). Data Envelopment Analysis: A Comprehensive Text with Models, Applications, References and DEASolver Software (Second Edition). Publisher: Springer US. https://doi.org/10. 1007/978-0-387-45283-8.

\section{Submit your manuscript to a SpringerOpen ${ }^{\circ}$ journal and benefit from:}

- Convenient online submission

- Rigorous peer review

- Open access: articles freely available online

- High visibility within the field

- Retaining the copyright to your article

Submit your next manuscript at $\boldsymbol{\nabla}$ springeropen.com 